\title{
In vivo magnetic resonance spectroscopy for ovarian cancer diagnostics: quantification by the fast Padé transform
}

\author{
Dževad Belkić1 ${ }^{1}$ Karen Belkić1,2,3
}

Received: 18 August 2016 / Accepted: 27 September 2016 / Published online: 2 November 2016 (C) The Author(s) 2016. This article is published with open access at Springerlink.com

\begin{abstract}
Early-stage ovarian cancer has an excellent prognosis, but due mainly to late detection, ovarian cancer remains a major cause of cancer deaths among women. In vivo magnetic resonance spectroscopy (MRS) would be an excellent candidate for early ovarian cancer detection, being non-invasive, surpassing anatomic imaging to identify metabolic features of cancer, and free of ionizing radiation. However, the present meta-analysis of 13 studies indicates that with conventional Fourier-based processing, in vivo MRS insufficiently distinguished 134 cancerous from 114 benign ovarian lesions. The fast Padé transform (FPT), an advanced signal processor with high-resolution and parametric (quantification-equipped) capabilities is best qualified for MRS time signals from the ovary, as demonstrated in our earlier proof-of-concept studies. We now apply the FPT to MRS time signals encoded in vivo on a $3 \mathrm{~T}$ scanner, echo time of $30 \mathrm{~ms}$, from a borderline serous cystic ovarian tumor. The FPT-produced total shape spectrum was better resolved than with Fourier processing. Spectra averaging through the FPT generated a denoised total shape spectrum. Subsequent parametric analysis reconstructed dense component spectra in the "usual" mode: absorption and dispersion components mixed and "ersatz" mode: reconstructed phases set to zero, thus eliminating interference effects. Numerous metabolites, including potential cancer biomarkers, were identified and quantified by the FPT, including isoleucine, valine, lipids, lactate, alanine, lysine, $N$-acetyl aspartate, $N$-acetylneuraminic acid, gluta-
\end{abstract}

Dževad Belkić

Dzevad.Belkic@ki.se

1 Department of Oncology-Pathology, Karolinska Institute, Building P-9, 2nd Floor, P.O. Box 260, Stockholm 17176, Sweden

2 School of Community and Global Health, Claremont Graduate University, Claremont, CA, USA

3 Institute for Prevention Research, Keck School of Medicine, University of Southern California, Alhambra, CA, USA 
mine, choline, phosphocholine, myoinositol. Many of these are difficult or impossible to detect with Fourier plus fitting techniques for in vivo MRS of the ovary. These Padé-generated results are promising, overcoming major barriers hindering MRS from becoming a key method for non-invasively assessing ovarian lesions.

Keywords Magnetic resonance spectroscopy - Ovarian cancer diagnostics · Mathematical optimization · Fast Padé transform

\section{Abbreviations}

\begin{tabular}{ll} 
Ace & Acetic acid \\
AcNeu & N-acetylneuraminic acid \\
Ala & Alanine \\
Av & Average \\
au & Arbitrary units \\
B & Benign ovarian lesion \\
Bet & Betaine \\
BL & Borderline ovarian lesion \\
BRCA1 & Breast cancer susceptibility gene 1 \\
BRCA2 & Breast cancer susceptibility gene 2 \\
BW & Bandwidth \\
CA & Carcinoma \\
CHESS & Chemical shift selective \\
Cho & Choline \\
CI & Confidence interval \\
Cit & Citrate \\
cm & Centimeter \\
COSY & Correlated spectroscopy \\
Cr & Creatine \\
Crn & Creatinine \\
CT & Computerized tomography \\
DFT & Discrete Fourier transform \\
DWI & Diffusion weighted imaging \\
E & Ersatz \\
FFT & Fast Fourier transform \\
FID & Free induction decay \\
FPT & Fast Padé transform \\
FWHM & Full width at half maximum \\
Iso & Isoleucine \\
GE & General Electric \\
Glc & Glucose \\
Gln & Glutamine \\
Glu & Glutamate \\
Gly & Glycine \\
GPC & Glycerophosphocholine \\
& \\
\hline &
\end{tabular}




\begin{tabular}{|c|c|}
\hline $\mathrm{HBOC}$ & Hereditary breast and ovarian cancer \\
\hline His & Histidine \\
\hline HLSVD & Hankel-Lanczos Singular Value Decomposition \\
\hline HRMAS & High resolution magic angle spinning \\
\hline IDFT & Inverse discrete Fourier transform \\
\hline IFFT & Inverse fast Fourier transform \\
\hline Iso & Isoleucine \\
\hline $\mathrm{L}$ & Liter \\
\hline Lac & Lactate \\
\hline LCModel & Linear combination of model in vitro spectra \\
\hline Leu & Leucine \\
\hline Lip & Lipid \\
\hline Lys & Lysine \\
\hline Mann & Mannose \\
\hline Met & Methionine \\
\hline METAS & Metastatic \\
\hline m-Ins & Myoinositol \\
\hline $\mathrm{mL}$ & Milliliter \\
\hline $\mathrm{mm}$ & Millimeter \\
\hline MR & Magnetic resonance \\
\hline MRI & Magnetic resonance imaging \\
\hline MRS & Magnetic resonance spectroscopy \\
\hline MRSI & Magnetic resonance spectroscopic imaging \\
\hline MW & Mann Whitney \\
\hline NAA & $N$-acetyl aspartate \\
\hline NEX & Number of excitations \\
\hline NPV & Negative predictive value \\
\hline NS & Statistically non-significant \\
\hline $\mathrm{OC}$ & Ovarian cancer \\
\hline OR & Odds ratio \\
\hline PC & Phosphocholine \\
\hline $\mathrm{PCr}$ & Phosphocreatine \\
\hline PLCO & Prostate, Lung, Colon, and Ovarian (trial) \\
\hline ppm & Parts per million \\
\hline PPV & Positive predictive value \\
\hline PRESS & Point resolved spectroscopy \\
\hline $\mathrm{Pt}$ & Patient \\
\hline Pts & Patients \\
\hline Pyr & Pyruvate \\
\hline RMS & Root-mean-square \\
\hline $\mathrm{S}$ & South \\
\hline SCSOCS & Shizuoka Cohort Study on Ovarian Cancer Screening \\
\hline $\mathrm{SD}$ & standard deviation \\
\hline SNR & Signal-noise ratio \\
\hline SNS & Signal-noise separation \\
\hline SRI & Spectral region of interest \\
\hline
\end{tabular}




$\begin{array}{ll}\text { STEAM } & \text { Stimulated echo acquisition method } \\ \text { SVD } & \text { Singular Value Decomposition } \\ \text { TE } & \text { Echo time } \\ \text { Thr } & \text { Threonine } \\ \text { TR } & \text { Repetition time } \\ \text { Tu } & \text { Tumor } \\ \text { TVUS } & \text { Transvaginal ultrasound } \\ \text { Tx } & \text { Treatment } \\ \text { Tyr } & \text { Tyrosine } \\ \text { U } & \text { Usual } \\ \text { UKCTOCS } & \text { UK Collaborative Trial of Ovarian Cancer Screening } \\ \text { Univ } & \text { University } \\ \text { Val } & \text { Valine } \\ \text { VOI } & \text { Volume or voxel of interest } \\ \text { WET } & \text { Water suppression through enhanced } T_{1} \text { effects } \\ \text { ww } & \text { Wet weight } \\ \text { Y } & \text { Year } \\ \text { 1D } & \text { One dimensional } \\ \text { 2D } & \text { Two dimensional } \\ \text { 3-HB } & \text { 3-Hydroxybutyrate } \\ \mu M & \text { Micromole }\end{array}$

\section{Introduction}

Mathematical optimization has a special relevance to the problem of ovarian cancer diagnostics. This is related to the application of advanced signal processing methods to evaluate data encoded via magnetic resonance spectroscopy (MRS). The anticipated added value of a high-resolution and clinically reliable signal processor to this problem would be improved accuracy for early detection of ovarian cancer. We begin by contextualizing the problem, briefly reviewing the relevant medical aspects, followed by our meta-analysis of the results to date that are based upon the conventional Fourier processing of in vivo MRS time signals encoded from the ovary. Then, we proceed to a succinct presentation of the advanced signal processing provided by the fast Padé transform (FPT). Thereby, we strive to provide the multi-disciplinary background for viewing the new results presented in this paper, applying the FPT to in vivo MRS time signals encoded from the ovary.

\subsection{Dimensions of the problem: impact and risk of ovarian cancer}

The ovary is a small, moving ellipsoid organ. Its normal mean volume in adult females ranges from 6.1 to $1.8 \mathrm{~cm}^{3}$ depending on age. Especially in early-stage cancer, the ovary may be only slightly enlarged or of normal size [1]. Cancers in this tiny organ are the sixth most often occurring malignancy among women throughout the world. In many parts of the world, including the U.S., Scandinavia and Israel, ovarian cancer is even more common, and in a number of countries the incidence of ovarian cancer 
appears to be increasing [2-5]. Ovarian cancer has a very high case fatality rate [6]. In the U.S. alone, over 14,000 women die each year from ovarian cancer [7].

Among the risk factors for ovarian cancer is heredity, which accounts for up to $25 \%$ of cases [8-12]. Familial ovarian cancer has been most widely identified in relation to the hereditary breast and ovarian cancer (HBOC) syndrome, with germline mutations in BRCA1 and BRCA2 tumor suppressor genes ${ }^{1}$ being responsible for the vast majority of HBOC. Several other gene mutations also appear to be associated with HBOC or other hereditary ovarian cancers. The Lynch syndrome characterized by non-polyposis colorectal cancer also includes increased risk of ovarian cancer, as well as endometrial cancer [9,12].

Non-hereditary risk factors for ovarian cancer include use of hormone replacement therapy $[11,13]$, unhealthy life-style (smoking, high-saturated fat diet intake, obesity) [11], late childbirth, nulliparity, endometriosis [14,15], and possibly exposure to diagnostic ionizing radiation, as well as to talc, pesticides or herbicides $[11,15]$. Night shift work may also increase the risk of ovarian cancer [16], possibly in relation to circadian genes that are highly expressed in the ovaries, since these genes regulate ovulation [17].

\subsection{Late detection of ovarian cancer}

The main reason for the high mortality is that the majority of ovarian cancers are detected late, at Stage III or IV with tumor spread outside the true pelvis [18]. When detected early, ovarian cancer has an excellent prognosis, especially at Stage Ia (confined to a single ovary) for which five-year survival rates are well above $90 \%$ [19]. The challenge, however, is that early stage ovarian cancer is most often asymptomatic and, as noted, the ovary may still be of normal size [1]. Once symptoms such as abdominal pain, bloating and urinary discomfort have appeared, the disease is often already in an advanced stage. Taking a more proactive approach towards symptoms has been suggested, i.e. performing more symptom-triggered diagnostic workups. However, it has not been demonstrated that this approach would contribute substantially to earlier diagnosis of ovarian cancer [20].

\subsubsection{Ultrasound and biomarkers}

Transvaginal ultrasound (TVUS) and serum cancer antigen (CA-125) ${ }^{2}$ have been the most commonly used diagnostic methods to screen for ovarian cancer. Recent evidence from a large, randomized controlled trial [the UK Collaborative Trial of Ovarian Cancer Screening (UKCTOCS)] indicates that, compared to women who received no

\footnotetext{
1 BRCA1 denotes the breast cancer susceptibility gene 1, BRCA2 denotes the breast cancer susceptibility gene 2. These are tumor suppressor genes that, when functioning normally, maintain cell growth at the proper rate. With harmful mutations of BRCA1 or BRCA2, cells have an increased chance of unchecked growth, and the risk of breast and ovarian cancer is increased.

2 Serum cancer antigen, CA-125 is a protein which, when present is often associated with ovarian cancer. However, it has poor sensitivity for early stage ovarian cancer and is also non-specific, being present in other malignancies as well as in a number of non-cancerous conditions, including pregnancy.
} 
screening, there may have been reduced mortality from ovarian cancer during years 7 to 14 of the study among the women who received annual multimodal screening with serum CA-125 interpreted with use of the "Risk of Ovarian Cancer Algorithm". It was considered, however, that further follow-up is needed before definitive conclusions could be reached concerning the efficacy and cost-effectiveness of ovarian cancer screening using this strategy [21]. In the large-scale Prostate, Lung, Colorectal and Ovarian (PLCO) Trial from the U.S., the combination of CA-125 and TVUS to screen for ovarian cancer in asymptomatic women did not reduce mortality. This strategy had associated harms related to a large percentage of false positive findings. Therefore, the conclusion was that this screening strategy should not be recommended [22]. Concordantly, the conclusion of a randomized multi-center study of 48027 women from Japan [Shizuoka Cohort Study on Ovarian Cancer Screening (SCSOCS)] was that TVUS plus CA-125 did not lead to a significant increase in stage I ovarian cancer detection among asymptomatic women who had passed menopause [23]. Notably, among the 40801 women enrolled in the SCSOCS who never had a CA-125 value above the upper limit of normal ( 35 Units/mL), some 4859 women had an abnormal TVUS. Among those women who subsequently underwent surgery, 8 ovarian cancers were diagnosed. The authors conclude: "surgery-detected ovarian cancer is not rare among women with CA-125 levels of 35 Units/mL or less-levels generally thought to be in the normal range" [24] (p. 133).

It has been suggested that certain other biomarkers could provide better diagnostic accuracy than CA-125 for early ovarian cancer detection [25-29]. However, none of these other biomarkers are considered to provide sufficient improvement in sensitivity and specificity to support their routine use for ovarian cancer screening [30].

A particular concern regarding the described screening strategies aimed at early ovarian cancer detection are the adverse consequences of false positive findings. These include poorer adherence to further screening recommendations, emotional distress, as well as a substantial percentage (15\% in the PLCO study) of serious complications among women undergoing surgical intervention for false positive results [31,32]. The impact of compromised subsequent fertility among women in their reproductive years who have undergone surgical intervention is also an important consideration. Overall, the major problem is that screening using TVUS and CA-125 can lead to many unnecessary surgical procedures [33], without an evident contribution to early detection and reduced mortality from ovarian cancer. In other words, for women who are not clearly at high risk for ovarian cancer, the "harms" of routine ovarian cancer screening are considered to outweigh the benefits [34].

\subsubsection{Possible added value of magnetic resonance imaging}

With the aid of magnetic resonance imaging (MRI), adnexal masses with morphologic characteristics that are indeterminate on TVUS can sometimes be better identified as benign or malignant, with a specific diagnosis facilitated in certain cases $[35,36]$. In a meta-analysis comparing three morphological imaging modalities, MRI was found to yield greater incremental value than computerized tomography (CT) for identifying ovarian cancer when the findings on TVUS were indeterminate [37]. Still, almost one-fourth of benign ovarian lesions were misdiagnosed as cancerous using TVUS 
with MRI as a second imaging technique [37]. Generally, MRI is highly sensitive, with ovarian cancer usually identified. The main problem is lack of specificity, such that non-malignant findings cannot consistently be distinguished from ovarian cancer, without further investigation. With the addition of diffusion-weighted imaging (DWI) further improvement in specificity can be gained, although a substantial number of false positive findings still occur $[38,39]$. There is also some evidence that DWI may be helpful in reflecting response of advanced stage ovarian cancer to neoadjuvant chemotherapy [40].

\subsection{In vivo magnetic resonance spectroscopy of the ovary: the present meta-analysis of published results}

Magnetic resonance imaging, MRI, provides high spatial resolution, such that morphology is very well visualized. Via magnetic resonance spectroscopy, MRS, it becomes possible to go beyond anatomy, to assess the metabolic features of tissues or organs. The molecular changes that typify the cancer process, i.e. the "hallmarks of cancer" [41] can potentially be revealed through MRS [42].

Several investigative teams have applied in vivo MRS with the aim of improving ovarian cancer diagnostics. We performed a systematic search ${ }^{3}$ of the literature and found published results for 134 malignant ovarian lesions, 114 benign ovarian lesions and 3 lesions of the ovary that were classified as "borderline" (BL), using in vivo proton MRS time signals encoded via clinical (1.5 or $3 \mathrm{~T}$ ) magnetic resonance (MR) scanners [40,43-55]. Appendix 1 provides a summary of the clinical aspects and design of these studies, with salient information concerning the reported MRS data. In all 251 cases, the nature of the ovarian lesion was subsequently confirmed histopathologically. As seen in column 3 of Appendix 1, there was much diversity in the histopathologic nature of these lesions. There was also substantial diversity in the MRS-related methodology, as described in column 5 of Appendix 1. In most of the studies, some assessment of spectral quality, notably, Signal-noise ratio (SNR) was reported, with these issues addressed in detail in Ref. [52]. The common methodologic feature of all these in vivo studies was that the MRS time signals were processed by the fast Fourier transform (FFT) as is the conventional practice, with eventual post-processing via fitting in some instances, such as in Refs. [40,45,51,52,54].

Column 6 of Appendix 1 summarizes the MRS findings. The main metabolite peaks resolved were: lipid (Lip) at $1.3 \mathrm{ppm}$ (parts per million), an inverted lactate (Lac) doublet also at around $1.3 \mathrm{ppm}$, creatine $(\mathrm{Cr})$ at $3.0 \mathrm{ppm}$, choline (Cho) at 3.2 ppm or total Cho between 3.14 and $3.34 \mathrm{ppm}$ and Lip at $5.2 \mathrm{ppm}$. In addition, a peak with resonance frequency between 2.0 and 2.1 ppm was reported by some authors [48$52,54]$. Based upon in vitro analysis, this peak was found to be comprised of $N$-acetyl aspartate (NAA) as well as $N$-acetyl groups from glycoproteins and/or glycolipids [50]. The NAA component may be a reflection of a molecular water pump [48]. Recall that

\footnotetext{
3 The search was performed using two reliable search engines for medicine: OVID MEDLINE and PUBMED. The search terms were magnetic resonance spectroscopy and (ovary or ovarian cancer). All identified published clinical studies using in vivo MRS to evaluate ovarian lesions were included in the meta-analysis. Bibliographies of relevant articles and personal files were also reviewed.
} 
in the healthy human brain, NAA is the tallest peak and this reflects the abundance and viability of neurons. Choline reflects phospholipid metabolism of cell membranes and is a marker of membrane damage, cellular proliferation and cell density, while $\mathrm{Cr}$ is a marker of energy metabolism. Anaerobic glycolysis is indicated by Lac. Most commonly, the metabolic information was described qualitatively (presence or absence of a given peak), and these are the data that could be pooled for meta-analysis. Various procedures were performed aimed at quantifying Cho. Due to the diversity of units as well as of the procedures, these data could not be pooled. Metabolite concentration ratios of Cho to $\mathrm{Cr}$ were reported in two of the studies [51,54]. In both studies, the $\mathrm{Cho/Cr}$ concentration ratio was significantly higher in cancerous ovarian lesions than in the benign ovaries. However, these data were given for individual patients in only one [54] of these studies, and thus, no data pooling could be done.

Univariate analysis of the pooled data for the 134 ovarian cancers and 114 benign ovarian lesions is presented in Table 1. It can be seen that Cho at $3.2 \mathrm{ppm}$ was significantly more often detected in cancerous compared to benign ovarian lesions. This was also the case for Lac at $1.3 \mathrm{ppm}$, but the number of patients for whom data on Lac were available was relatively small. There was no significant difference between cancerous and benign ovarian lesions regarding detection of Lip at $1.3 \mathrm{ppm}$. Data concerning an NAA peak at $\sim 2.0 \mathrm{ppm}$ almost exclusively indicated its presence, not absence. Altogether an NAA peak at $\sim 2.0$ ppm was reportedly detected in 46 of the cancerous and 36 of the benign ovarian lesions. This would not be a statistically significant difference, even if all the missing data actually indicated absence of an NAA peak (Yates $\chi^{2}=0.10, p=0.747$ ). The majority of the MRS analyses for both the benign and malignant ovarian lesions were on voxels containing solid tissue. In an additional analysis (not shown in Table 1) comparing the metabolic findings among the ovarian cancers alone, Cho at $3.2 \mathrm{ppm}$ was detected more often in the voxels containing solid tissue compared to those containing cystic material (Fisher's exact test, 2-tailed, $p=0.00000)$.

The patients with ovarian cancer were significantly older than those with benign ovarian lesions, although there were much missing data for this variable (age), particularly among the patients with ovarian cancer. Magnetic field strength, $B_{0}$, and echo time (TE) were similar for the ovarian cancers and the benign ovarian lesions. In an additional analysis (not shown in Table 1), there was a borderline significant association between $B_{0}$ and Cho detection (Pearson $\chi^{2}=3.97, p=0.046$, Yates $\left.\chi^{2}=3.19, p=0.074\right)$, but no association between $B_{0}$ and Lac detection.

Table 2 displays the present results of logistic regression analysis, from which the positive predictive value (PPV), negative predictive value (NPV) and overall accuracy of several models were determined. In the left column of Table 2, the unadjusted models are shown. These indicate how well Cho at $3.2 \mathrm{ppm}$ (top panels), Lac at 1.3 ppm (middle panels) as well as Cho and Lac together (bottom panels) predict whether the ovarian lesions were cancerous or benign. Based on the detection of Cho alone, some 50 benign ovarian lesions would be incorrectly classified as cancerous, i.e. as false positive results, indicated by the PPV of $66 \%$. Some 20 malignant ovarian lesions would be incorrectly considered benign based upon lack of detected Cho, i.e. false negative results, with an NPV of 57.4\%. Lactate alone provided better PPV and NPV with a statistically more significant model, but data were available for only $25 \%$ of the 
Table 1 Univariate analysis comparing ovarian cancer and benign ovarian lesions

\begin{tabular}{|c|c|c|c|}
\hline & Ovarian cancer $N=134$ & Significance & Benign ovarian lesions $N=114$ \\
\hline & Number (\%) & & Number $(\%)$ \\
\hline \multicolumn{4}{|c|}{ Metabolites detected by in vivo MRS } \\
\hline \multicolumn{4}{|c|}{ Cho (3.2 ppm) } \\
\hline Present & $97(82.9)$ & $p=0.007$ & $50(64.9)$ \\
\hline Absent & $20(17.1)$ & & $27(35.1)$ \\
\hline Missing data & 17 & & 37 \\
\hline \multicolumn{4}{|l|}{ Lip (1.3 ppm) } \\
\hline Present & $44(67.7)$ & NS & $57(59.4)$ \\
\hline Absent & $21(32.3)$ & & 39 (40.6) \\
\hline Missing data & 69 & & 18 \\
\hline \multicolumn{4}{|l|}{ Lac (1.3 ppm) } \\
\hline Present & $18(69.2)$ & $p=0.0006$ & $8(22.2)$ \\
\hline Absent & $8(30.8)$ & & $28(77.8)$ \\
\hline Missing data & 108 & & 78 \\
\hline \multicolumn{4}{|l|}{ Lesion type } \\
\hline Solid & $85(80.2)$ & NS & $49(72.1)$ \\
\hline Cystic & $21(19.8)$ & & $19(27.9)$ \\
\hline \multirow[t]{2}{*}{ Missing data } & 28 & & 46 \\
\hline & Mean $\pm \mathrm{SD}$ (range) & & Mean $\pm \mathrm{SD}$ (range) \\
\hline Patient age & $56.3 \pm 7.8(43-81)$ & $p=0.000000$ & $41.9 \pm 15.2(22-84)$ \\
\hline Missing data & 88 & & 46 \\
\hline Magnetic field strength $B_{0}$ & $1.89 \pm 0.66(1.5-3.0)$ & NS & $1.87 \pm 0.65(1.5-3.0)$ \\
\hline Missing data & 2 & & 9 \\
\hline TE (in ms) & $122 \pm 31.5(30-144)$ & $\mathrm{NS} \zeta$ & $107.5 \pm 46.7(30-144)$ \\
\hline Missing data & 0 & & 0 \\
\hline
\end{tabular}

Comparisons for the categorical variables assessed by Pearson $\chi^{2}$ test with Yates' continuity correction. Means are compared with two-sample "t" tests, unless indicated as $\zeta$ for MW test

All significant levels 2 sided, SD (standard deviation), NS (Statistically non-significant)

Analysis performed using Statistica 64 software

patients. We also used an unadjusted logistic regression model including both Cho and Lac, which was more powerful than the models for either of the two metabolites alone. In the unadjusted model including both Cho and Lac, a higher PPV was attained, but 3 fewer cases were included than for the model with Lac alone.

The logistic regression models with adjustment for age and $B_{0}$ are presented on the right column of Table 2. Age was included since, as seen in Table 1, there was a significantly greater likelihood that the ovarian lesion was cancerous with increased age of the patient. As noted, Cho was more likely to be detected with higher $B_{0}$. We therefore included the variable $B_{0}$ in these adjusted models. The adjusted logistic regression model for Cho provided a better NPV than did the unadjusted model, 


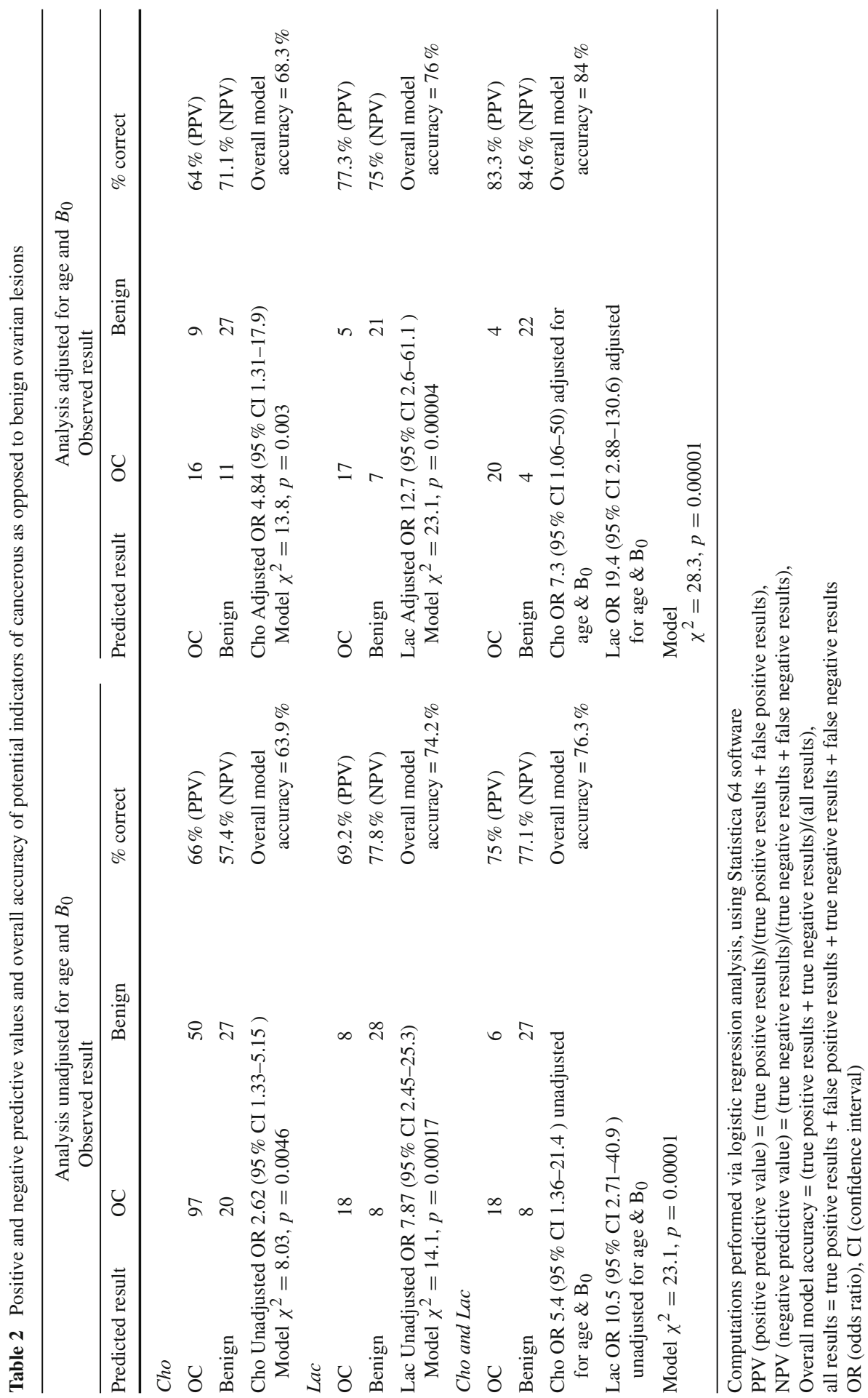


although with markedly fewer patients included. There were twelve fewer patients in the adjusted than in the unadjusted Lac model, with only marginal improvement in overall accuracy. The adjusted model with both Lac and Cho, also with a total of fifty patients, provided the best PPV, NPV and overall accuracy. In that model Lac and Cho each had the highest odds ratios (OR), but their $95 \%$ confidence intervals (CI) were the widest. Even with this strongest logistic regression model, four of twentyfour patients with benign ovarian lesions were incorrectly predicted to have ovarian cancer, and four of twenty-six patients with ovarian cancer were erroneously predicted to have benign lesions.

Overall, it can be concluded that while some insights are gleaned, in vivo MRS has not yet provided sufficient distinction between cancerous and benign ovarian lesions. Due to motion artefacts plus the small size of the ovary, encoding good quality MRS time signals is technically very difficult. As noted, current practice is to rely upon the FFT to convert the encoded MRS time signal into its spectral representation in the frequency domain. The FFT is a low resolution, non-parametric signal processor. It cannot reliably separate out the noise that corrupts the recorded MRS time signal, such that poor SNR is a major problem when using clinical MR scanners. Yet, there are many MR-observable compounds that characterize malignant versus benign ovarian lesions using in vitro MRS [50,56-62].

\subsection{In vitro MRS findings from the ovary}

Greater possibilities emerge for distinguishing malignant from benign ovarian lesions by in vitro MRS. Not only can much stronger static magnetic fields be applied, but the methods of analytical chemistry can also be used on the excised specimens. Generally much better resolution is achieved compared to in vivo MRS in which time signals are most often encoded with 1.5 or $3 \mathrm{~T}$ clinical scanners.

Using linear discriminant analysis training with leave-one-out (12 normal, 22 cancer) for evaluating 7 normal versus 15 ovarian cancer specimens, normal and benign lesions were identified according to six discriminating peaks: $1.47 \mathrm{ppm}$ (fatty acid), $1.68 \mathrm{ppm}$ [lysine (Lys)], $2.80 \mathrm{ppm}$ (fatty acid), $2.97 \mathrm{ppm}$ (Cr), $3.17 \mathrm{ppm}$ (Cho) and $3.34 \mathrm{ppm}$ (taurine), with $95 \%$ sensitivity and $86 \%$ specificity [56]. In an investigation of 19 normal or benign ovarian samples, 3 with BL pathology and 37 ovarian cancers [57], amplitude ratios of peaks at $0.9 \mathrm{ppm}$ (Lip methyl), $1.3 \mathrm{ppm}$ (Lip methylene), 1.7 ppm (Lys and polyamines) and $3.2 \mathrm{ppm}$ (Cho), distinguished normal or benign samples from BL and malignant ovarian samples with a sensitivity of $95 \%$ and specificity of $86 \%$.

Examining samples from 9 malignant and 19 benign ovarian cysts, Massuger et al. [58] reported higher concentrations of Lac, as well as alanine (Ala), isoleucine (Iso), leucine (Leu), methionine (Met) and valine (Val) in the cancer specimens. However, the ranges overlapped. Higher pyruvate (Pyr) and 3-hydroxybutyrate (3-HB) were observed in the cancerous ovarian cyst fluid, attributed to the rapid cellular metabolism which leads to elevations in 3-HB. The high concentrations of Iso, Leu, and Val, as branched chain amino acids, were considered to be protein breakdown products associated with proteolysis and necrosis. There was also an endometrioma located 
in the adnexal region included in the study, for which much higher levels of Ala, Iso, Lys, Met, Val, threonine (Thr), as well as glycine were seen compared to the malignant ovarian cysts. Using one-dimensional (1D) and two-dimensional (2D) in vitro MRS, the investigation was extended to 12 cancerous and 23 benign ovarian cysts [59]. Therein, concentrations of Iso (1.02 ppm), Val (1.04 ppm), Thr (1.33 ppm), Lac (1.41 ppm), Ala (1.51 ppm), Lys (1.67-1.78 ppm), Met (2.13 ppm), as well as glutamine (Gln) (2.42-2.52 ppm) and Cho (3.19 ppm) were all significantly higher in the malignant cyst fluid. There was, however, some overlap between the individual values of each of these metabolite concentrations in the cancerous and the benign samples.

For the borderline, BL, serous cystic ovarian lesion assessed with in vivo MRS at TE $=30$ and $136 \mathrm{~ms}$ in Ref. [50], as noted, the cystic fluid was also evaluated in vitro. Using 1D spectral analysis, in addition to the Lac doublet at $\sim 1.3 \mathrm{ppm}$, several resonances appeared in the region around $1.0 \mathrm{ppm}$, most likely corresponding to Iso and Val, inter alia. Alanine was seen at $\sim 1.45 \mathrm{ppm}$ and acetate (Ace) at $\sim 1.9 \mathrm{ppm}$. A broad resonance centered at $2.06 \mathrm{ppm}$ was observed, which disappeared when the deproteinized cyst fluid was reanalyzed. In contrast, however, a singlet resonance at $2.03 \mathrm{ppm}$ assigned to NAA was seen in the deproteinized benign serous ovarian cyst fluid from three other patients. Further analysis was performed on the native BL ovarian cystic fluid, using 2D correlated spectroscopy (COSY). Cross peaks of two different types of bound sialic i.e. $N$-acetylneuraminic (acNeu) acid were seen. It was considered that these resonances may be attributed to $N$-acetylated glycoproteins and/or glycolipids. These findings underscore the importance of assessing the underlying components of resonant peaks, rather than relying solely upon total shape spectra.

In a subsequent study, this group of investigators applied Gas ChromatographyMass Spectrometry to ovarian cystic fluid [60]. In the nine epithelial ovarian tumors with a serous histology (serous cystadenocarcinoma), it was reported that NAA was generally present in high micromolar concentrations. Since the serum concentrations of NAA were usually low in these patients, it was suggested that NAA was produced locally in these ovarian tumors. Other histological types of cystadenocarcinomas (mucinous, endometrioid and clear cell), however, mainly showed low micromolar concentrations of NAA, and these concentrations were similar to those in the serum. The authors suggested that the high levels of NAA may be associated with the water accumulation within the tumors and thereby could contribute to the formation of large serous cystic lesions.

An extensive metabolomic assessment using high resolution magic angle spinning (HRMAS) was made of 31 epithelial ovarian carcinomas (22 serous, 4 endometrioid, and 5 mucinous carcinomas), 5 BL ovarian tumors and 3 samples from healthy ovarian tissue [61]. Pattern differences were observed among the malignant subtypes, with NAA being characteristic of serous carcinomas, and $N$-acetyl-lysine of mucinous carcinomas. Among four patients with poor survival, higher levels of Val, Leu and Lys were found.

Most recently, metabolomic analysis was reported of 23 ovarian cyst fluid samples ( 8 benign, $5 \mathrm{BL}$ and 10 cancerous) via proton MRS [62]. These authors examined a wide chemical shift region from about 0.8 to $8.2 \mathrm{ppm}$. Included therein were the "Aliphatic region" from about 1.8 to $4.2 \mathrm{ppm}$, and the "Aromatic region" from about 
6.6 to $8.0 \mathrm{ppm}$. The latter contained histidine, tyrosine and phenylalanine. At 8.2 ppm, a hypoxanthine resonance was detected only in a sub-cohort of the ovarian cancers. In the aliphatic region, the citrate (Cit) level was found to be elevated in the benign tumors. Lys was associated with the malignant ovarian lesions. Choline and Lac were reportedly found in progressively higher levels in benign versus BL versus malignant ovarian lesions. It was suggested that different metabolic patterns might help distinguish various histological types of cancerous ovarian cystic fluid, and that cancer biomarkers as well as therapeutic targets might be identified through this methodology.

There have also been some in vitro MRS studies of cells obtained from ovarian cancer and benign ovarian lesions. Via proton MRS, only mean intracellular Lac at $1.32 \mathrm{ppm}$ was found to be significantly higher in cells obtained from ovarian cancers as compared to benign ovarian cysts; the total Cho level was marginally significantly higher in the cancer cells [63]. The chemical shift region between 3.20 and $3.24 \mathrm{ppm}$ has been examined in more detail, comparing human epithelial ovarian carcinoma cell lines with normal or immortalized ovarian epithelial cells, with phosphocholine (PC) found to be three- to eight-fold higher in the cancer cells [64]. It has thus been suggested that there may be possibilities for improving the diagnostic yield of MRS for ovarian cancer by identifying the components of total Cho, in particular PC, which is a recognized biomarker of malignant transformation [65].

\subsection{More advanced signal processing applied to MRS data from the ovary: results to date}

The described results for in vivo and in vitro MRS have been based upon conventional signal processing via the fast Fourier transform, FFT. This occurred because MR scanners have the FFT built-in, such that generally the MRS time signals are automatically processed to yield the total shape spectra from which metabolite peaks, or resonances are visually identified. Reliance upon the FFT for analysis of MRS time signals has limited the diagnostic yield especially of in vivo MRS for ovarian cancer. The main reasons for this limited yield are that the FFT is a low resolution, non-parametric processor which can only provide total shape spectra. Most of the total shape spectra generated by the FFT from in vivo MRS time signals encoded from the ovary are quite rough, often with very poor SNR.

By contrast, the fast Padé transform, FPT, an advanced signal processor with highresolution capabilities and parametric (quantification-equipped) features [66-69], is particularly amenable to processing MRS time signals from the ovary. In the FPT, the spectrum is given by a non-linear response function as the unique ratio of two polynomials. The high resolution of the FPT is due to extrapolation and interpolation capabilities, as well as to its nonlinearity which is responsible for noise suppression. The FPT has two variants: the $\mathrm{FPT}^{(+)}$and $\mathrm{FPT}^{(-)}$that, by definition, converge inside and outside the unit circle, respectively. Moreover, the $\mathrm{FPT}^{(+)}$and $\mathrm{FPT}^{(-)}$are also convergent in their complementary domains (outside and inside the unit circle, respectively) by way of the Cauchy analytical continuation. For example, the $\mathrm{FPT}^{(-)}$ for $|z|>1$ (outside the unit circle) is an accelerator of the already existing conver- 
gence of the input series given by the Green function in the harmonic variable $z^{-1}$. On the other hand, since this latter input series diverges inside the unit circle, the $\mathrm{FPT}^{(+)}$ for $|z|<1$ converts a divergent into a convergent series via the said powerful concept of the Cauchy analytical continuation [70].

We will herein highlight the results obtained to date applying the FPT to synthesized MRS time signals similar to those encoded from benign and cancerous ovary. In the next sub-section, a succinct overview of the mathematical properties of the FPT will be given.

The first applications of the FPT were to synthesized noiseless time signals associated with MRS data for benign and cancerous ovarian cyst fluid from Ref. [59]. Including the closely-lying resonances, such as Iso (1.023 ppm) and Val (1.042 ppm), all the input spectral parameters were correctly reconstructed thereby, for each of the twelve true metabolites. With only 64 signal points $(N / 16)$ of the full time signal $N=1024$, the metabolite concentrations were accurately computed $[66,71]$. These results remained stable at longer signal lengths. In sharp contradistinction, at $N_{\mathrm{P}}=64$, the FFT generated very crude spectra that were completely uninterpretable and necessitated 8192 signal points to coarsely resolve all 12 resonances. Even then, a number of the peak heights were not correct in the FFT. For full convergence of the absorption total shape spectra for the noiseless data from benign and malignant ovary, the FFT required 32768 signal points [66,71]. These results [66,71] clearly showed the superior resolving power of the FPT. For these noiseless ovarian data, besides reconstructing the twelve physical resonances, at convergence, some twenty non-physical resonances were also generated. Via the Signal-noise separation (SNS) procedure associated with the FPT [72] (see next sub-section), these spurious resonances were identified by their pole-zero confluences and zero amplitudes, and were discarded. Subsequently, the FPT was applied to simulated MRS time signals associated with ovarian cancer, for which noise was added with standard deviation $\sigma=0.01156$ RMS, where RMS is the root-mean-square of the noise-free time signal [73-75]. With this added noise, convergence required 128 signal points $(N / 8, N=1024)$ for correct reconstruction of the spectral parameters for the twelve physical resonances, with the additional 52 spurious resonances identified by their pole-zero confluences and the associated zero-valued amplitudes [73]. The pole-zero coincidence was not always complete in the $\mathrm{FPT}^{(-)}$with higher levels of noise $(\sigma=0.1156 \mathrm{RMS}, \sigma=0.1296 \mathrm{RMS}$ and $\sigma=0.2890 \mathrm{RMS}$ ) and near zero amplitudes rather than actual zero amplitudes were observed for some of the spurious resonances [74,75]. Since genuine metabolites could be present at low concentrations, the peak amplitudes could be very small, as well. Thus, we raised the question as to how to be entirely certain which resonances are nonphysical and which are genuine? We emphasized that this question is vitally important when proceeding from proof-of-principle studies with synthesized, i.e. theoretically generated input data to encoded time signals for which the number of resonances and their parameters are not known prior to spectral analysis.

The so-called stability test was shown to be a practical strategy for the FPT. Namely, by varying the partial signal length and/or by adding more noise, there was a set of resonances (even with very small peak heights) that showed a pronounced stability. Therefore, they were classified as physical (genuine). By contrast, with the same type of the mentioned perturbations, another set of resonances was identified that exhibited 
marked instability. Hence, these latter resonances were categorized as non-physical (spurious) $[74,75]$.

Detailed analysis has been recently performed [76] on the synthesized noisecorrupted benign ovarian cyst data based upon the encoded time signals from Ref. [59]. All the genuine resonances were clearly identified by both FPT variants, $\mathrm{FPT}^{( \pm)}$, with the correct metabolite concentrations computed at short total signal lengths. It should be noted that for the final analysis, both the $\mathrm{FPT}^{(+)}$and $\mathrm{FPT}^{(-)}$are always used for cross-checking. Signal-noise separation, SNS, was most efficient in the $\mathrm{FPT}^{(+)}$ where the genuine and spurious resonances were segregated inside and outside the unit circle, respectively. In the $\mathrm{FPT}^{(-)}$, both these resonance types were mixed and were all located outside the unit circle. Pole-zero coincidence of spurious resonances remained complete in the $\mathrm{FPT}^{(+)}$, with a denoised spectrum produced automatically for these MRS data from benign ovary. These latter findings were deemed particularly advantageous for practical applications as needed for the in vivo setting.

\subsection{Mathematical features of the fast Padé transform of particular relevance for MRS}

\subsubsection{The limitations of Fourier-based processing of MRS time signals}

As noted, clinical scanners use the fast Fourier transform, FFT, to convert the encoded time signal or free induction decay (FID) into its spectral representation in the frequency domain. The Fourier spectrum is expressed as a single polynomial:

$$
\text { FFT: } F_{m}=\sum_{n=0}^{N-1} c_{n} \exp (-2 \pi i m n / N), \quad 0 \leq m \leq N-1
$$

where $2 \pi m / T$ is the fixed $m$ th Fourier grid frequency, and $\left\{c_{n}\right\}$ is the set of complexvalued time signal points. Here, $T$ is the total signal duration or total acquisition time, $T=N \tau$, where $N$ is the total signal length and $\tau$ is the sampling time (dwell time, sampling rate), which is the inverse of the bandwidth (BW). The variables $\exp ( \pm 2 \pi i m n / N)$ are the undamped sinusoids and cosinusoids $(n m \tau / T=n m / N)$. Note also that the time signal can be reproduced exactly (including all the intact noise) from the Fourier spectrum via the inverse Fourier transform (IFFT):

$$
\text { IFFT: } \quad c_{n}=\frac{1}{N} \sum_{m=0}^{N-1} F_{m} \exp (2 \pi i m n / N), \quad 0 \leq n \leq N-1 .
$$

When the signal lengths are in the composite form, $N=2^{m}(m=1,2,3, \ldots)$, the FFT algorithm is a rapid processor, which is the main reason for its widespread usage. Whenever $N$ is non-composite, i.e. any positive integer, the FFT and IFFT from (1a) and (1b) become, respectively, the discrete Fourier transform (DFT) and the inverse DFT (IDFT). 
The low resolution capabilities of Fourier processing stem, in part, from the fact that the Fourier-generated total shape spectrum is obtained from pre-assigned frequencies with a minimal separation fixed by the total acquisition time, $T$, without any possibilities for interpolation based on the analyzed $N$ data points of the FID. In attempting to improve resolution, SNR inevitably worsens, since with longer $T$, the FID will have decayed such that predominantly noise will be recorded. This is especially problematic with low field (1.5 and 3 T) MR clinical scanners [68]. Further contributing to poor resolution and SNR are the linearity of the Fourier method, and its lack of extrapolation capabilities. As to the latter, no information can be gleaned beyond the final encoded signal point, $\mathrm{c}_{N-1}$. The customary zero-filling of $\left\{c_{n}\right\}(0 \leq n \leq N-1)$, e.g. to double the total FID length $N$, cannot improve resolution. This is the case because the original $N$ data points in the FID already contain the entire information. As such, zero-filling or zero-padding can only serve to somewhat improve the visual presentation of the ensuing FFT spectrum, albeit with sinc-type artificial oscillations along the baseline. However, the said improved visual effect is irrelevant for quantitative analysis which is the main task of MRS.

Recall that only a total shape spectrum (envelope) can be produced with the Fourier analysis, since this is exclusively a non-parametric estimator. The subsequent step is often post-processing via fitting. Thereby, the number of resonances included in a given model is actually just a mere speculation, from which estimates of metabolite concentrations are frequently biased and inaccurate, as discussed in Ref. [67].

\subsubsection{How these limitations are overcome by the fast Padé transform}

In sharp contrast to the FFT, the fast Padé transform, FPT, is optimally suited for processing MRS time signals $[67,77]$. As noted, the FPT-produced spectrum is a nonlinear response function via the unique ratio of 2 polynomials. In the diagonal form, this spectrum is $P_{K} / Q_{K}$, where $K$ is the polynomial degree (also called the model order). The fixed Fourier mesh $2 \pi m \tau / T(m=0,1,2, \ldots)$ is not necessary in the FPT, such that the spectrum can be computed at any sweep frequency. Since resolution is not limited by the total acquisition time, $T$, there is no conundrum between increasing $T$ in attempts to improve resolution at the expense of deterioration of SNR. Moreover, the FPT can extrapolate beyond $T$ via the unique polynomial quotient $P_{K} / Q_{K}$, extracted directly from the investigated FID. This is opposed to the FFT, which, as noted, is limited by a sharp cut-off of the FID at the end of the acquisition time. The nonlinearity of the FPT further contributes to its high resolution properties by suppressing noise, thus improving SNR $[67,68,77]$. The presence of the numerator $\left(P_{K}\right)$ and denominator $\left(Q_{K}\right)$ polynomials aids in noise cancellation from the Padé spectrum $P_{K} / Q_{K}$. This occurs because both $P_{K}$ and $Q_{K}$ contain a similar amount of noise imported from $\left\{c_{n}\right\}$ by the expansion coefficients of these two polynomials. Note, when e.g. two observables $A$ and $B$ are measured in an experiment, or theoretically generated by numerical computations with finite precision, errors in $A$ and $B$ are substantially cancelled in the ratio $A / B$, which is frequently used for data interpretation and analysis.

The number of physical metabolites as well as their spectral parameters, the fundamental frequencies $\left\{\omega_{k}\right\}$ and the associated amplitudes $\left\{d_{k}\right\}$ in the set $\left\{\omega_{k}\right.$, $\left.d_{k}\right\}(1 \leq k \leq K)$ of the specified time signal $\left\{c_{n}\right\}(0 \leq n \leq N-1)$, are accurately 
reconstructed by the FPT. Consequently, metabolite concentrations are correctly computed thereby $[69,77]$. The angular (or cyclic) frequencies $\omega_{k}$ are connected to the linear frequencies $\nu_{k}$ by $\omega_{k}=2 \pi v_{k}$ (with the like relation also existing for the sweep frequencies, $\omega=2 \pi \nu$ ).

The infinite-rank Green function $G\left(z^{-1}\right)$ gives the exact response function. This is defined by the Maclaurin series with the time signal points $\left\{c_{n}\right\}$ as the expansion coefficients:

$$
G\left(z^{-1}\right)=\sum_{n=0}^{\infty} c_{n} z^{-n}, \quad z=\mathrm{e}^{i \tau \omega} \quad \text { (Exact Green series). }
$$

It should be noted, however, that in realistic situations, only a finite number $N(N<$ $\infty)$ of signal points $\left\{c_{n}\right\}$ is available. A truncated response function must then be provided, as the finite-rank Green function given by the Green polynomial $G_{N}\left(z^{-1}\right)$ :

$$
G_{N}\left(z^{-1}\right)=\sum_{n=0}^{N-1} c_{n} z^{-n} \quad(\text { Exact Green polynomial })
$$

In the terminology of analysis of discrete time series, these infinite- and finiterank Green functions can also be termed the infinite and finite $z$-transform [67]. In the $\mathrm{FPT}^{( \pm)}$, the input response function $G_{N}\left(z^{-1}\right)$ from (3) is approximated by the Green-Padé functions $G_{K}^{ \pm}\left(z^{ \pm 1}\right)$, as the diagonal rational polynomials in the harmonic variables $z^{ \pm 1}$ :

$$
\begin{array}{r}
G_{N}\left(z^{-1}\right) \approx G_{K}^{-}\left(z^{-1}\right)=\frac{P_{K}^{-}\left(z^{-1}\right)}{Q_{K}^{-}\left(z^{-1}\right)} \equiv \frac{\sum_{r=0}^{K} p_{r}^{-} z^{-r}}{\sum_{s=0}^{K} q_{s}^{-} z^{-s}} ; \\
\mathrm{FPT}^{(-)} \text {(Anti-causal Green-Padé function), }
\end{array}
$$

$$
\begin{aligned}
& G_{N}(z) \approx G_{K}^{(+)}(z)=\frac{P_{K}^{+}(z)}{Q_{K}^{+}(z)} \equiv \frac{\sum_{r=1}^{K} p_{r}^{+} z^{r}}{\sum_{s=0}^{K} q_{s}^{+} z^{s}} ; \\
& \mathrm{FPT}^{(+)} \text {(Causal Green-Padé function) } .
\end{aligned}
$$

Here, $\left\{p_{r}^{ \pm}\right\}$and $\left\{q_{s}^{ \pm}\right\}$are the expansion coefficients of the polynomials $P_{K}^{ \pm}$and $Q_{K}^{ \pm}$, respectively. Notice, that the linear term $p_{0}^{+}$is absent from the expansion for $G_{K}^{(+)}(z)$, i.e. $p_{0}^{+} \equiv 0$.

The expansion coefficients $\left\{p_{r}^{ \pm}, q_{s}^{ \pm}\right\}$of the numerator $P_{K}^{ \pm}\left(z^{ \pm 1}\right)$ and denominator $Q_{K}^{ \pm}\left(z^{ \pm 1}\right)$ polynomials are extracted uniquely from the time signal points $\left\{c_{n}\right\}$ by solving a single system of linear equations from definitions (4) and (5), respectively. The spectra $P_{K}^{ \pm}\left(z^{ \pm 1}\right) / Q_{K}^{ \pm}\left(z^{ \pm 1}\right)$ from the $\mathrm{FPT}^{( \pm)}$can also be expressed in the canonical forms:

$$
\frac{P_{K}^{ \pm}\left(z^{ \pm 1}\right)}{Q_{K}^{ \pm}\left(z^{ \pm 1}\right)}=\frac{p_{K}^{ \pm}}{q_{K}^{ \pm}} \prod_{k=1}^{K} \frac{z^{ \pm 1}-z_{k, P}^{ \pm}}{z^{ \pm 1}-z_{k, Q}^{ \pm}}
$$


The solutions of the characteristic equations, $P_{K}^{ \pm}\left(z^{ \pm 1}\right)=0$ and $Q_{K}^{ \pm}\left(z^{ \pm 1}\right)=0$, have the roots denoted by $z_{k, P}^{ \pm 1} \equiv z_{k, P}^{ \pm}$and $z_{k, Q}^{ \pm 1} \equiv z_{k, Q}^{ \pm}(1 \leq k \leq K)$, respectively. Here, we relabel $z_{k}^{+}$as $z_{k, Q}^{+}$, where the second subscript $Q$ is introduced to indicate that this harmonic variable is the root of the denominator polymial $Q_{K}^{+}(z)$. This is done in order to distinguish this variable from the corresponding root $z_{k, P}^{+}$of the numerator polynomial $P_{K}^{+}(z)$. Complex variables $\left\{z_{k, Q}^{+}\right\}$are constituents of the fundamental harmonics in the sets $\left\{z_{k, Q}^{ \pm}, d_{k}^{ \pm}\right\}$. The fundamental amplitudes $d_{k}^{ \pm}$are the Cauchy residues of the spectra $P_{K}^{ \pm}\left(z^{ \pm 1}\right) / Q_{K}^{ \pm}\left(z^{ \pm 1}\right)$ taken at the fundamental harmonics $z^{ \pm 1} \equiv z_{k, Q}^{ \pm}$. When the roots are non-degenerate, i.e. representing simple poles alone, these amplitudes are:

$$
d_{k}^{ \pm}=\frac{P_{K}^{ \pm}\left(z_{k, Q}^{ \pm}\right)}{Q_{K}^{ \pm \prime}\left(z_{k, Q}^{ \pm}\right)}, \quad Q_{K}^{ \pm^{\prime}}\left(z^{ \pm 1}\right)=\frac{\mathrm{d}}{\mathrm{d} z^{ \pm 1}} Q_{K}^{ \pm}\left(z^{ \pm 1}\right), \quad 1 \leq k \leq K .
$$

The equivalent expressions for $d_{k}^{ \pm}$derived from (7) are:

$$
d_{k}^{ \pm}=\frac{p_{K}^{ \pm}}{q_{K}^{ \pm}} \prod_{k^{\prime}=1}^{K} \frac{z_{k, Q}^{ \pm}-z_{k^{\prime}, P}^{ \pm}}{\left(z_{k, Q}^{ \pm}-z_{k^{\prime}, Q}^{ \pm}\right)_{k^{\prime} \neq k}} .
$$

Each numerator on the rhs of (8) is proportional to $z_{k, Q}^{ \pm}-z_{k^{\prime}, P}^{ \pm}\left(k, k^{\prime}=1, \ldots, K\right)$. Consequently, the amplitudes $d_{k}^{ \pm}$are proportional to the pole-zero distance (a metric):

$$
d_{k}^{ \pm} \propto z_{k, Q}^{ \pm}-z_{k, P}^{ \pm}
$$

Therefore, the Cauchy residue reflects the behavior of a line integral of a meromorphic function around a specified pole. Reconstruction of the $2 K$ complex fundamental parameters $\left\{\omega_{k, Q}^{+}, d_{k}^{ \pm}\right\}$is thereby completed.

The expressions for $G_{K}^{(-)}\left(z^{-1}\right)$ and $G_{K}^{(+)}(z)$ from (4) and (5) approximate the Green function $G_{N}\left(z^{-1}\right)$. The Green function $G\left(z^{-1}\right)$ from (2) is convergent outside the unit circle $(|z|>1)$, and divergent inside the unit circle $(|z|<1)$. The convergence rate of $G_{K}^{(-)}\left(z^{-1}\right)$ is faster than the original Maclaurin series, such that the $\mathrm{FPT}^{(-)}$is an accelerator of convergence. The convergence radii $R_{N}$ and $R_{K}^{-}$of $G_{N}\left(z^{-1}\right)$ and $G_{K}^{-}\left(z^{-1}\right)$ are both non-zero $R_{N} \neq 0$ and $R_{K}^{-} \neq 0$, respectively. In addition, the latter is larger than the former $R_{K}^{-}>R_{K}$.

In contrast, the $\mathrm{FPT}^{(+)}$via $G_{K}^{(+)}(z)$ uses the variable $z$ and converges inside the unit circle $(|z|<1)$ which is where the exact Green function $G\left(z^{-1}\right)$ diverges. As stated, by the Cauchy concept of analytical continuation, the $\mathrm{FPT}^{(+)}$converts a divergent into a convergent series. The convergence radius $R_{K}^{+}$of $G_{K}^{+}(z)$ is markedly changed from being exactly zero $\left(R_{N}=0\right.$ for $|z|<1$ for $G_{N}$ as $\left.N \rightarrow \infty\right)$ to $R_{K}^{+}>0$. In this way, the $\mathrm{FPT}^{(+)}$extends the validity of the response function (spectrum) to $|z|<1$ where the input Green series $G\left(z^{-1}\right)$ and polynomial $G_{N}\left(z^{-1}\right)$ do not exist, due to their divergence inside the unit circle. 
It follows from the definitions (4) and (5) that the same truncation level of $N$ would have a very different effect in the input polynomial $G_{N}\left(z^{-1}\right)$ and the Padé rational polynomials $P_{K}^{ \pm}\left(z^{ \pm 1}\right) / Q_{K}^{ \pm}\left(z^{ \pm 1}\right)$. Namely, if in (2) we truncate $n$ to e.g. $M=N / 2$, the resulting spectrum $G_{M}\left(z^{-1}\right)$ would be based only on the $N / 2$ time signal points. On the other hand, e.g. for the $\mathrm{FPT}^{(-)}$, the same truncation of $n$ to $N / 2$ in (2), which is transferred to (4), would generate the spectrum $P_{K}^{-}\left(z^{-1}\right) / Q_{K}^{-}\left(z^{-1}\right)$ which includes the information from the entire non-truncated set $\left\{c_{n}\right\}(0 \leq n \leq N-1)$. Such an occurrence comes from the fact that expanding $P_{K}^{-}\left(z^{-1}\right) / Q_{K}^{-}\left(z^{-1}\right)$ in its Maclaurin series (in power of $z^{-1}$ ) would reconstruct exactly the full input $N$ time signal points $\left\{c_{n}\right\}(0 \leq n \leq N-1)$. This remarkable property of the rational Padé polynomials explains why the $\mathrm{FPT}^{(-)}$is able to achieve a better resolution that the FFT by using the same number of FID data points. Likewise, the FPT ${ }^{(-)}$can achieve the same resolution as that of the FFT by using fewer signal points. These features that are rooted in definition (4) for the $\mathrm{FPT}^{(-)}$have also been systematically observed in the explicit computations by this variant of the FPT. Relative to the FPT ${ }^{(-)}$, the other variant, i.e. the $\mathrm{FPT}^{(+)}$, usually requires more signal points (a more over-determined system of linear equations for the polynomial expansion coefficients) because it must induce convergence into the divergent input expansion of $G\left(z^{-1}\right)$ or $|z|<1$.

$\mathrm{The} \mathrm{FPT}^{(+)}$and $\mathrm{FPT}^{(-)}$are complementary. When full convergence is achieved in the $\mathrm{FPT}^{( \pm)}$, internal cross-validation is provided thereby. In other words, at the end of the analysis, we obtain $\omega_{k, Q}^{+} \approx \omega_{k, Q}^{-} \approx \omega_{k}$ and $d_{k}^{+} \approx d_{k}^{-} \approx d_{k}$, where $\left\{\omega_{k}, d_{k}\right\}$ are the complex frequencies and amplitudes from the input time signal $c_{n}$ or FID which reads as:

$$
c_{n}=\sum_{k=1}^{K} d_{k} \mathrm{e}^{i n \tau \omega_{k}}, \quad 0 \leq n \leq N-1 \quad \text { (Input Time Signal or FID). }
$$

Having solved the quantification problem by the $\mathrm{FPT}^{( \pm)}$, the complex total shape spectra from (4)-(6) can alternatively be computed by using the Heaviside partial fraction expansions:

$$
\frac{P_{K}^{ \pm}\left(z^{ \pm 1}\right)}{Q_{K}^{ \pm}\left(z^{ \pm 1}\right)}=\frac{p_{0}^{ \pm}}{q_{0}^{ \pm}}+\sum_{k=1}^{K} \frac{d_{k}^{ \pm} z^{ \pm 1}}{z^{ \pm 1}-z_{k, Q}^{ \pm}} \quad \text { (Heaviside Partial Fractions) }
$$

where $p_{0}^{+}=0$, as in (5).

Quantification in MRS is actually the harmonic inversion problem. The $\mathrm{FPT}^{(+)}$and $\mathrm{FPT}^{(-)}$solve this problem exactly, using only the equidistantly sampled time signal $\left\{c_{n}\right\}$ to reconstruct all the complex fundamental frequencies and amplitudes $\left\{\omega_{k}, d_{k}\right\}$, from (10).

\subsubsection{Signal-noise separation}

Since poles are the only singularities of the $\mathrm{FPT}^{( \pm)}$, the spectra $P_{K}^{ \pm}\left(z^{ \pm 1}\right) / Q_{K}^{ \pm}\left(z^{ \pm 1}\right)$ are meromorphic functions. The poles $\left\{z_{k, Q}^{ \pm}\right\}$and zeros $\left\{z_{k, P}^{ \pm}\right\}$of these spectra are the 
roots of $Q_{K}^{ \pm}\left(z^{ \pm 1}\right)=0$ and $P_{K}^{ \pm}\left(z^{ \pm 1}\right)=0$, respectively. The spectral poles and zeros provide the physical parameters of the system which generated the time signals as a response to an external excitation. The amplitudes are also directly related to the spectral poles and zeros since $d_{k}^{ \pm} \propto z_{k, Q}^{ \pm}-z_{k, P}^{ \pm}$, according to (9).

The poles that are stable against external perturbations are physical. On the other hand, the poles that oscillate widely when exposed even to minimal perturbation are unstable and, hence, unphysical. In addition, unstable poles never converge and, thus, behave like noise, i.e. as random fluctuations.

By examining the spectral poles and zeros in the $\mathrm{FPT}^{( \pm)}$, stable versus unstable poles can be identified. For stable structures, the poles and zeros do not coincide, $z_{k, Q}^{ \pm} \neq z_{k, P}^{ \pm}$, i.e. they are distinct. Contrarily, the unstable structures display pole-zero confluence, i.e. $z_{k, Q}^{ \pm} \approx z_{k, P}^{ \pm}$, and are called Froissart doublets. Genuine resonances $\left(z_{k, Q}^{ \pm} \neq z_{k, P}^{ \pm}\right)$have non-zero amplitudes $\left(d_{k}^{ \pm} \neq 0\right)$, since $d_{k}^{ \pm} \propto z_{k, Q}^{ \pm}-z_{k, P}^{ \pm}$, as per (9). On the other hand, spurious resonances $\left(z_{k, Q}^{ \pm}=z_{k, P}^{ \pm}\right.$or $z_{k, Q}^{ \pm} \approx z_{k, P}^{ \pm}$) have zero or nearly zero amplitudes $\left(d_{k}^{ \pm}=0\right.$ or $d_{k}^{ \pm} \approx 0$ ).

After the model order $K$ in $P_{K}^{ \pm} / Q_{K}^{ \pm}$has stabilized, such that all the physical resonances have been reconstructed, computing the Padé spectra for a higher degree polynomial, $K+m(m=1,2,3, \ldots)$, would only generate more non-physical resonances. These spurious resonances would exhibit pole-zero coincidence $\left(z_{k, Q}^{ \pm}=z_{k, P}^{ \pm}\right)$ and, hence, $d_{k}^{ \pm}=0$ for $k=K+m(m=1,2,3, \ldots)$. All the numerator and denominator terms with spurious poles and spurious zeros in the canonical forms from (6) would cancel each other. This occurs in all the representations of the $\mathrm{FPT}^{( \pm)}$, be it the non-parametric spectra $P_{K}^{ \pm}\left(z^{ \pm 1}\right) / Q_{K}^{ \pm}\left(z^{ \pm 1}\right)$ (computed directly from this ratio at any sweep frequency $v$ ) or their parametric counterparts from the canonical forms in (6) or via the Heaviside partial fractions in (11). As such, pole-zero cancellations lead to stabilization of the computed spectra:

$$
\frac{P_{K+m}^{ \pm}\left(z^{ \pm 1}\right)}{Q_{K+m}^{ \pm}\left(z^{ \pm 1}\right)}=\frac{P_{K}^{ \pm}\left(z^{ \pm 1}\right)}{Q_{K}^{ \pm}\left(z^{ \pm 1}\right)} \quad(m=1,2,3, \ldots)
$$

Moreover, with Padé reconstruction the number of physical resonances, i.e. the number of fundamental harmonics $K$ is treated as an unknown, and its value must also be found. This occurs when the reconstructed frequencies and amplitudes have converged. In other words, the running (or sweep) model order $K^{\prime}$ (or equivalently, the degree $K^{\prime}$ of the Padé rational polynomials $P_{K^{\prime}}^{ \pm} / Q_{K^{\prime}}^{ \pm}$) for which the reconstructed frequencies and amplitudes have stabilized, would be the exact number $K$ of harmonics contained in the input FID from (10).

In summary, by gradually increasing the degree of the Padé polynomials until the genuine frequencies and amplitudes stabilize, any further increase in the polynomial degree generates exclusively spurious resonances. The latter are identified by their instability as well as by their pole-zero confluences that yield zero or near-zero amplitudes. This procedure is termed "Signal-noise separation", SNS, and has been thoroughly validated within MRS for noiseless as well as noise-corrupted synthesized time signals $[72,78]$. 
Most recently, the mechanism of SNS has been shown analytically [79]. Therein, the exact reconstruction of the input time signal and spectrum was demonstrated by eliciting, via an analytical derivation, two key features: pole-zero coincidence and near-zero amplitude of a Froissart doublet, which is a pole-zero spurious pair. The mathematical formalism was provided, showing precisely how a pole-zero coincidence yields pole-zero cancellation in the reconstructed spectrum.

This stabilization by way of its mechanism of pole-zero cancellation is a key feature of the $\mathrm{FPT}^{( \pm)}$. It is unique to the $\mathrm{FPT}^{( \pm)}$because of the special form of the rational polynomials $P_{K}^{ \pm} / Q_{K}^{ \pm}$for the Padé spectra. It is in this latter quotient of two polynomials that pole-zero coincidence can occur first, and then, pole-zero cancellation follows. Overall, detection of Froissart doublets via pole-zero confluences yielding the stabilization of the Padé spectra represents a veritable signature that the entire information from the input time signal has been exhausted by the $\mathrm{FPT}^{( \pm)}$. And, it is in this remarkable way that the $\mathrm{FPT}^{( \pm)}$can reconstruct exactly all the physical parameters $\left\{\omega_{k}, d_{k}\right\}$ from the examined FID in (10).

\subsection{Other proof-of-principle studies for benchmarking the FPT}

In addition to the described results applying the FPT to theoretically synthesized FID data, reminiscent of the time signals encoded from cancerous and benign ovary, detailed proof-of-principle studies have also been performed using MRS data from other tissues. The FPT has been demonstrated to accurately quantify MRS time signals, yielding quantitative information for a large number metabolites from cancerous, benign and/or normal brain, breast and prostate as shown in studies on simulated time signals [69,77,80-88]. In investigations applying the FPT to synthesized FIDs that were akin to those encoded in vivo from the brain of a healthy volunteer at $1.5 \mathrm{~T}$, proofof-concept validation was provided. Exact reconstruction of the spectral parameters was accomplished, and thereby the concentrations of metabolites were correctly computed. This was the case for very closely overlapping resonances with chemical shifts differing by a mere 0.001 ppm or less $[69,77,81]$.

A further proof-of-principle MRS study was carried out on the time signals encoded from the standard General Electric (GE) phantom head [89]. Through detailed inspection of the convergence process via "parameter averaging", the accuracy and stability of the Padé-reconstructed spectral parameters, i.e. the complex-valued fundamental frequencies $\left\{\omega_{k, Q}^{+}\right\}$and the associated amplitudes $\left\{d_{k}^{+}\right\}$, were confirmed, even for those resonances that were very closely overlapping.

\subsection{The fast Padé transform for processing in vivo encoded MRS time signals: results to date}

Besides the previous studies on synthesized FIDs, and the FIDs encoded from the GE phantom, the FPT has also been used to process MRS time signals encoded in vivo on a 1.5 T MR scanner from pediatric brain tumor [90], as well as from pediatric cerebral asphyxia $[79,91]$ and from healthy adult brain [92]. Studies applying the FPT were also done on MRS time signals encoded from healthy adult human brain using higher 
field MR scanners (4 and 7 T) [67,68,77,93-95]. In all these studies, the resolution of total shape spectra was markedly superior with the FPT, compared to the FFT.

Most notable has been the parametric capability of the FPT, particularly in spectrally dense chemical shift regions, where very closely-overlapping resonances were resolved and quantified. Among these identified were cancer biomarkers and other diagnostically important metabolites [77,79,90,91].

\subsubsection{Water suppression}

Suppression of the residual (and still giant) water resonance is a major practical challenge for in vivo MRS. In our recent work $[79,90,91]$ applying the $\mathrm{FPT}^{(+)}$to in vivo MRS time signals encoded from the brain via a 1.5 T MR scanner, suppression of the water residual has been an important consideration. The conventional procedure for residual water suppression has used the Hankel-Lanczos Singular Value Decomposition (HLSVD) which includes fitting by artificial resonances that produce more spuriousness in addition to the already noisy MRS time signal. Via a step function with the non-parametric FPT, we introduced an information-preserving procedure for suppressing residual water by windowing [90]. It was verified that this windowing procedure did not affect the spectral components within the spectral region of interest (SRI). There were some non-essential effects at the edges outside the SRI when comparing the water residual suppressed and unsuppressed Padé reconstructions. Since the full equivalence of the non-parametrically and parametrically generated total shape spectra in the $\mathrm{FPT}^{(+)}$was confirmed, we subsequently chose the latter, employing only the components $\left(P_{K}^{+} / Q_{K}^{+}\right)_{k}$ with chemical shifts from the SRI selected in a way which avoids the giant residual water resonance [79]. These parametrically generated envelopes via $P_{K}^{+} / Q_{K}^{+}$were computed using the Heaviside partial fraction sum (11). Thus, with the parametric $\mathrm{FPT}^{(+)}$and appropriate choice of SRI, the water residual suppression problem can be completely overcome without the need for windowing. The same procedure has been verified to work with the $\mathrm{FPT}^{(-)}$, as well.

\subsubsection{Iterative averaging: a stabilization procedure through the FPT}

The iterative averaging procedure was recently introduced as a powerful and efficient strategy for regularizing spectra against the destabilizing effect of changes in the sought model order $K$. This was implemented using in vivo MRS time signals encoded from the brain with a $1.5 \mathrm{~T}$ clinical scanner $[79,91]$. The initial step yielding the 1 st average envelope can be viewed as a counterpart to "signal averaging" which is routinely carried out by averaging about 200 encoded FIDs in the time domain to improve SNR. It is seen that for different model orders $K$, many large noise-like spikes appear in the spectra. Using a sequence of values of the model order $K$, the 1st average envelope (arithmetic average) is produced. Further, this complex envelope is inverted (by way of the IFFT or IDFT) to generate the 1st reconstructed FID. Such an FID is subjected to the FPT to obtain the next set of envelopes for the same sequence of values of $K$ as considered in the previous iteration. This new sequence of envelopes is averaged and the outlined procedure can be repeated until the prescribed accuracy of the reconstructed 
spectral parameters has been attained. With each iteration, there is a progressively greater level of suppression of spurious spectral structures that are very sensitive to changes in $K$. Not only the total shape spectra, but all four Padé-reconstructed spectral parameters for each genuine resonance also showed progressively reduced fluctuations with consecutive iterations. Convergence was thereby achieved, such that the values of spectral parameters were fully stabilized to the minimal level of deviation consistent with stochasticity [79].

Our overall conclusion from these most recent in vivo studies $[79,90,91]$ was that this multi-faceted Padé-based strategy for processing the dense spectra of the brain could vitally improve not only pediatric neurodiagnostics, but that a much wider range of clinical applications could also become within reach. Among these are areas of cancer diagnostics where in vivo MRS could potentially have the greatest added value. We have singled out early ovarian cancer diagnostics, where the need for an effective in vivo MRS-based screening method has been highlighted for nearly two decades $[58,76,96]$.

\subsection{Aim of the present study}

Exhaustive proof-of-concept testing on synthesized data has shown the FPT to be a super high resolution processor of MRS time signals, providing exact quantification of metabolites that can distinguish benign from malignant tissue. Particularly detailed benchmarking studies with the FPT using synthesized FIDs for benign and cancerous ovarian cyst fluid have been made. Since then, the FPT has been extensively validated for in vivo MRS time signals encoded using MR clinical scanners. Through the Padé methodology, solutions have been provided to major problems associated with in vivo MRS, such as how to most effectively handle the giant residual water resonance and how to achieve full stabilization of total shape spectra as well as of spectral parameters. In the present study, our aim is to apply the FPT for the first time to in vivo MRS time signals encoded from the ovary. We anticipate that through the fast Padé transform, much richer metabolic information will be gleaned from in vivo MRS encoded from ovarian tissue (especially at shorter echo times) than what has heretofore been the case using the conventional Fourier-based processing. The possibility for more effective early detection of ovarian cancer is the over-arching motivation for this line of investigation.

\section{Methods}

\subsection{Acquisition of the MRS time signal from a borderline serous cystic ovarian lesion}

The encoded FID data were kindly provided by our colleagues from the Department of Obstetrics and Gynecology, Radboud University Nijmegen Medical Center in the Netherlands. These data are from a 56 year-old patient with an enlarged left ovary as detected on TVUS, and who was included in their in vivo MRS study [50] (see also the present meta-analysis, Appendix 1). A 3 T Magnetom Tim Trio, Siemens clinical 
scanner was used to encode the MRS time signals, each of which contained 1024 data points. The bandwidth, BW, was $1200 \mathrm{~Hz}$, and the Larmor frequency was $v_{\mathrm{L}}=$ 127.732 MHz corresponding to the magnetic field strength $B_{0}=3$ tesla $\left(B_{0}=3 \mathrm{~T}\right)$. The sampling time $\tau$ was $0.833 \mathrm{~ms}(\tau=1 / \mathrm{BW} \approx 0.833 \mathrm{~ms})$.

Single-voxel proton MRS with the point-resolved spectroscopy sequence (PRESS) was used, with the voxel of interest $(3 \mathrm{~cm} \times 3 \mathrm{~cm} \times 3 \mathrm{~cm})$ in the inferior cystic part of the tumor. The repetition time (TR) was $2000 \mathrm{~ms}$ and two echo times, TE $=30$ and 136 ms were employed in the study of Kolwicjk et al. [50]. A total of 64 encoded FIDs were averaged to improve SNR. In other words, in the standard terminology within MRS, the number of excitations (NEX) was 64. In the present paper, we examine only the time signals encoded at $30 \mathrm{~ms}$. In Ref. [50], the giant water peak was partially suppressed through encoding via WET (water suppression through enhanced $T_{1}$ effects) and water non-suppressed FIDs were also encoded for referencing. The residual water content from the encoded FIDs was not further suppressed nor removed in the present study. Subsequent to the in vivo MRS encoding, the ovarian tumor was surgically removed and the diagnosis on histopathology was a borderline serous cystic lesion [50].

\subsection{Reconstructions using the encoded MRS time signal}

The encoded FIDs were processed with the DFT and the FPT. The FID phase was not corrected for the zero-order phase $\varphi_{0}$.

\subsubsection{Comparison of the DFT and the FPT: non-parametric reconstructions}

Via (1a), the spectra were computed in the DFT, since non-composite partial signal lengths $N_{\mathrm{P}}$ were employed $\left(N_{\mathrm{P}} \neq 2^{m} ; m=0,1,2, \ldots\right)$. Both variants, the $\mathrm{FPT}^{(+)}$ and $\mathrm{FPT}^{(-)}$, were used for the non-parametric Padé-based reconstructions. All the envelopes from the $\mathrm{FPT}^{(-)}$were reproduced by the $\mathrm{FPT}^{(+)}$.

The expansion coefficients of the polynomials $P_{K}^{ \pm}$and $Q_{K}^{ \pm}$in the $\mathrm{FPT}^{( \pm)}$are calculated from the time signal $\left\{c_{n}\right\}$ using the definitions in (4) and (5). After this initial step of the analysis, the non-parametrically computed total shape spectra are obtained at a given set of real-valued sweep frequencies $v$. In the present computations, the envelopes were evaluated at 1024 sweep frequencies $\nu$. Insofar as the phases $\varphi_{k}^{ \pm}$of the reconstructed FID amplitudes $d_{k}^{ \pm}=\left|d_{k}^{ \pm}\right| \exp \left(i \varphi_{k}^{ \pm}\right)$were all equal to zero, $\varphi_{k}^{ \pm}=0(1 \leq k \leq K)$, the real and imaginary parts of the complex spectra, i.e. $\operatorname{Re}\left(P_{K}^{ \pm} / Q_{K}^{ \pm}\right)$and $\operatorname{Im}\left(P_{K}^{ \pm} / Q_{K}^{ \pm}\right)$, would be purely absorptive and dispersive, respectively. In every encoded MRS time signals, however, the phases $\varphi_{k}$ of the FID amplitudes $d_{k}$ are mostly non-zero $\left(\varphi_{k} \neq 0\right)$, due to dephasing which takes place during encoding. Thus, the reconstructed values $\varphi_{k}^{ \pm}$are also such that $\varphi_{k}^{ \pm} \neq 0$. Consequently, absorption and dispersion lineshapes are mixed in both $\operatorname{Re}\left(P_{K}^{ \pm} / Q_{K}^{ \pm}\right)$and $\operatorname{Im}\left(P_{K}^{ \pm} / Q_{K}^{ \pm}\right)$.

\subsubsection{Averaging procedure}

We herein employ the method of the arithmetic averaging of spectra, which has been demonstrated to overcome a major obstacle for harmonic inversion, namely, 
over-sensitivity of spectra (envelopes, components) as well as of the fundamental frequencies and amplitudes to changes in model order $K$ [79,91]. Spectra averaging was previously done with three [91] and nine [79] iterations for the purpose of benchmarking this stabilization procedure. Presently, the iterative averaging of spectra has also been performed during the test computations. It turned out that already the 1 st average envelope provided very accurate reconstructions. Therefore, in the current work, it suffices to place the main focus on the 1st averaging of spectra. As done in Ref. [91], prior to averaging, we shall use the non-parametric $\mathrm{FPT}^{(+)}$to generate a number of envelopes for a range of $K$.

Recall, that in the Padé rational functions, as per (12), the spurious resonances cancel out with stabilization for systematically and gradually increased polynomial degree $K+m(m=1,2,3, \ldots)$. The mechanism for this is rooted in pole-zero cancellations, which occurs because spurious resonances display coincidence or near-coincidence of their poles and zeros [79]. These confluences (Froissart doublets) render spurious resonances highly unstable, particularly for changes in the model order $K$. Each envelope $P_{K+m}^{+}(z) / Q_{K+m}^{+}(z)(m=1,2,3, \ldots)$ will display different spuriousness due to random distributions of spurious poles and zeros in the complex frequency plane.

The complex 11 usual envelopes $\left(P_{K}^{+} / Q_{K}^{+}\right)^{\mathrm{U}}$ are computed for $K=575-625$, in increments of 5 from the FID encoded at TE $=30 \mathrm{~ms}$. Subsequently, we take the arithmetic average of these 11 envelopes, with the result indicated by $\left\{\mathrm{FPT}^{(+)}\right\}_{\mathrm{Av}}^{\mathrm{U}}$. We use the subscript Av to indicate the Average (Av). The complex average envelope $\left\{\mathrm{FPT}^{(+)}\right\}_{\mathrm{Av}}^{\mathrm{U}}$ is then subjected to the IDFT to generate a new FID, to which the parametric $\mathrm{FPT}^{(+)}$is applied. In Refs. [79,91], the increment $\Delta K$ for equidistantly augmenting the value of $K$ was equal to unity, $\Delta K=1$. With such a small $\Delta K$, some 31 envelopes for $K \in[375,415]$ were generated for iterative averaging [79,91]. Alternatively, in the present study, a larger interval for $K$ is chosen to be spanned, i.e. $K \in[575,625]$, but with a larger increment, $\Delta K=5$, for generation of 11 envelopes for spectra averaging. Any two adjacent spikes are likely to be more markedly different from each other in the case with $\Delta K=5$ than for $\Delta K=1$.

\subsubsection{Parametric processing with the $F P T^{(+)}$}

The results for the $\mathrm{FPT}^{(+)}$alone will be presented for parametric processing (quantification). As noted, cross-checking was performed throughout with the $\mathrm{FPT}^{(-)}$. The zeros and poles of the Padé spectrum, $P_{K}^{+} / Q_{K}^{+}$are given, respectively, by the roots of the characteristic equations of the numerator $\left(P_{K}^{+}\right)$and denominator $\left(Q_{K}^{+}\right)$polynomials. This is the main step in quantification within the $\mathrm{FPT}^{(+)}$, where Padé-based parametric processing is carried out via polynomial rooting. As mentioned, the Padé spectrum is a meromorphic function because $P_{K}^{+} / Q_{K}^{+}$has only polar singularities, namely, the zeros of $Q_{K}^{+}$are the poles of $P_{K}^{+} / Q_{K}^{+}$[67]. The fundamental frequencies $\omega_{k, Q}^{+}=[1 /(i \tau)] \ln \left(z_{k, Q}^{+}\right)$were generated through the roots of equation $Q_{K}^{+}(z)=0$. The amplitudes $d_{k}^{+}$were reconstructed via (7) [67]. 


\subsubsection{Modes of the component spectra}

The component spectra will be shown in two different modes. We begin with the "usual" (U) component spectra where the absorption and dispersion lineshapes are mixed together. Recall that the amplitudes $\left\{d_{k}^{+}\right\}(1 \leq k \leq K)$ are all complex-valued because their phases $\varphi_{k}^{+}$are non-zero. In chemical shift regions with many closelylying or overlapping resonances, the "absorption" components frequently appear as skewed Lorentzians. Employing the "ersatz" (E) mode signifies that the reconstructed phases $\varphi_{k}^{+}$have been set "by hand" to zero, and consequently, $\varphi_{k}^{+} \equiv 0(1 \leq k \leq K)$. Interference effects are thereby eliminated, such that purely absorptive Lorentzians are produced. These are often helpful for visual inspection. The "ersatz" and the "usual" mode of the component spectra for the $k$ th resonance are, respectively, given by:

$$
\begin{aligned}
& \left(\frac{P_{K}^{+}(z)}{Q_{K}^{+}(z)}\right)_{k}^{\mathrm{E}} \equiv \frac{\left|d_{k}^{+}\right| z}{z-z_{k, Q}^{+}} \quad(\text { Ersatz component } k) \\
& \left.\left(\frac{P_{K}^{+}(z)}{Q_{K}^{+}(z)}\right)_{k}^{\mathrm{U}} \equiv \frac{d_{k}^{+} z}{z-z_{k, Q}^{+}} \quad \text { (Usual component } k\right) .
\end{aligned}
$$

Via setting $\varphi_{k}^{+}=0$, we can return from (14) to (13) by replacing $d_{k}^{+} \equiv$ $\left|d_{k}^{+}\right| \exp \left(i \varphi_{k}^{+}\right)$with $\left|d_{k}^{+}\right|$. We reemphasize that the usual form $\operatorname{Re}\left(P_{K}^{+} / Q_{K}^{+}\right)_{k}^{\mathrm{U}}$ extracted from (14) contains both absorption and dispersion modes. Contrary to this, the real part of the component shape spectrum of the ersatz form $\operatorname{Re}\left(P_{K}^{+} / Q_{K}^{+}\right)_{k}^{\mathrm{E}}$ deduced from (13) is entirely in the absorption mode [90]. When juxtaposing the plots for $\operatorname{Re}\left(P_{K}^{+} / Q_{K}^{+}\right)_{k}^{\mathrm{U}}$ and $\operatorname{Re}\left(P_{K}^{+} / Q_{K}^{+}\right)_{k}^{\mathrm{E}}$, the peak position $\operatorname{Re}\left(v_{k, Q}^{+}\right)$needs to be properly understood. Suppose, for example, that $\operatorname{Re}\left(P_{K}^{+} / Q_{K}^{+}\right)_{k}^{\mathrm{U}}$ is a dispersive Lorentzian. It will be mapped to an absorptive Lorentzian in $\operatorname{Re}\left(P_{K}^{+} / Q_{K}^{+}\right)_{k}^{\mathrm{E}}$. The peak position $\operatorname{Re}\left(v_{k, Q}^{+}\right)$of this latter absorption mode for $\operatorname{Re}\left(P_{K}^{+} / Q_{K}^{+}\right)_{k}^{\mathrm{E}}$ will be located between the two lobes of the usual dispersive component $\operatorname{Re}\left(P_{K}^{+} / Q_{K}^{+}\right)_{k}^{\mathrm{U}}$. On the other hand, in cases when $\operatorname{Re}\left(P_{K}^{+} / Q_{K}^{+}\right)_{k}^{\mathrm{U}}$ is in the absorption mode, the positions, i.e. chemical shifts $\operatorname{Re}\left(v_{k, Q}^{+}\right)$of both the usual and ersatz peaks would coincide.

In the $\mathrm{FPT}^{(+)}$, the $T_{2}^{*}$-relaxation time for the $k$ th component is denoted by $T_{2 k}^{*+}$. This is related to the imaginary part of the reconstructed complex frequency $\omega_{k, Q}^{+}$or $v_{k, Q}^{+}$via $T_{2 k}^{*+}=1 /\left\{\operatorname{Im}\left(\omega_{k, Q}^{+}\right)\right\}=1 /\left\{2 \pi \operatorname{Im}\left(v_{k, Q}^{+}\right)\right\}$. This quantity is employed in the expressions for the peak heights $\left(H_{k}^{+}\right)^{\mathrm{U}}$ and $\left(H_{k}^{+}\right)^{\mathrm{E}}$, respectively, as:

$$
\begin{aligned}
\left(H_{k}^{+}\right)^{\mathrm{U}} & \equiv \frac{d_{k}^{+}}{D_{k}^{+}},\left(H_{k}^{+}\right)^{\mathrm{E}} \equiv \frac{\left|d_{k}^{+}\right|}{D_{k}^{+}} ; \quad D_{k}^{+}=1-\exp \left(-\tau / T_{2 k}^{*+}\right), \quad 0<D_{k}^{+}<1, \\
\operatorname{Re}\left(H_{k}^{+}\right)^{\mathrm{U}} & =\frac{\left|d_{k}^{+}\right|}{D_{k}^{+}} \cos \left(\varphi_{k}^{+}\right)=\left(H_{k}^{+}\right)^{\mathrm{E}} \cos \left(\varphi_{k}^{+}\right),
\end{aligned}
$$


where $\left(H_{k}^{+}\right)^{\mathrm{E}}$ is real-valued. There is also another expression, $H_{k}^{\mathrm{c}+} \equiv\left|d_{k}^{+}\right| /$ $\left(\tau \operatorname{Im} \omega_{k, Q}^{+}\right)$, for the peak heights $H_{k}^{\mathrm{c}+}$ of an absorptive conventional Lorentzian, which is directly expressed via $\omega$ instead of $z$ as $\left|d_{k}^{+}\right|\left\{\left(\operatorname{Im} \omega_{k, Q}^{+}\right) / \tau\right\} /\left\{\left(\omega-\omega_{k, Q}^{+}\right)^{2}+\right.$ $\left.\left(\operatorname{Im} \omega_{k, Q}^{+}\right)^{2}\right\}$. This latter result can also be deduced from (15) for narrow resonances with long relaxation times. Namely, for small $\tau / T_{2 k}^{*+}$, the series expansion for $\exp \left(-\tau / T_{2 k}^{*+}\right)$ yields $D_{k}^{+} \approx 1-\left(1-\tau / T_{2 k}^{*+}+\cdots\right) \approx \tau / T_{2 k}^{*+}=\tau \operatorname{Im} \omega_{k, Q}^{+}$. It follows from (15), therefore, that $\left|d_{k}^{+}\right| / D_{k}^{+} \approx\left|d_{k}^{+}\right| /\left(\tau \operatorname{Im} \omega_{k, Q}^{+}\right)=H_{k}^{\mathrm{c}+}$.

As per (5), the explicit expressions for the numerator $\left(P_{K}^{+}\right)$and denominator $\left(Q_{K}^{+}\right)$ polynomials in (13) and (14), are given by:

$$
P_{K}^{+}(z)=\sum_{r=1}^{K} p_{r}^{+} z^{r}, Q_{K}^{+}(z)=\sum_{s=0}^{K} q_{s}^{+} z^{s} .
$$

Therein, $\left\{p_{r}^{+}\right\}$and $\left\{q_{s}^{+}\right\}$are the expansion coefficients with $p_{0}^{+} \equiv 0$. In the FPT, we can use either the total signal length $N$ or the partial signal length $N_{\mathrm{P}}$. Insofar as the number $N_{\mathrm{P}}$ of the employed FID points is even, we would have $K=N_{\mathrm{P}} / 2$. The expansion coefficients $\left\{q_{s}^{+}\right\}$for the polynomial $Q_{K}^{+}(z)$ are extracted by solving the system of linear equations $\sum_{s=0}^{K} q_{s}^{+} c_{s^{\prime}+s}=0$ stemming from (5). The solutions $\left\{q_{s}^{+}\right\}$are subsequently refined via Singular Value Decomposition (SVD). After the set $\left\{q_{s}^{+}\right\}$becomes available, the expansion coefficients $\left\{p_{r}^{+}\right\}$in $P_{K}^{+}$are computed from the analytical expression $p_{r}^{+}=\sum_{r^{\prime}=0}^{K-r} c_{r^{\prime}} q_{r^{\prime}+r}^{+}$. The free term, $q_{0}^{+}$can be set to e.g. 1 or -1 without affecting the spectra or the spectral parameters $\left\{\omega_{k, Q}^{+}, d_{k}^{+}\right\}(1 \leq k \leq K)$ reconstructed by the $\mathrm{FPT}^{(+)}$. Likewise in the $\mathrm{FPT}^{(-)}$, a similar pair of equations exist for generating the expansion coefficients $\left\{p_{r}^{-}, q_{s}^{-}\right\}$with $p_{0}^{-} \neq 0[67,77]$. Overall, the two different algorithms in the $\mathrm{FPT}^{( \pm)}$are both simple and efficient. This is because the only numerical work involved is to solve a single system of linear equations for the expansion coefficients $\left\{q_{S}^{+}\right\}$or $\left\{q_{s}^{-}\right\}$and subsequently to root the characteristic polynomials $Q_{K}^{+}$or $Q_{K}^{-}$to reconstruct the fundamental frequencies $\left\{\omega_{k, Q}^{+}\right\}$or $\left\{\omega_{k, Q}^{-}\right\}$in the $\mathrm{FPT}^{(+)}$or $\mathrm{FPT}^{(-)}$. Unlike the HLSVD which generates the amplitudes by solving yet another system of linear equations, the $\mathrm{FPT}^{(+)}$and $\mathrm{FPT}^{(-)}$provide $\left\{d_{k}^{ \pm}\right\}$from the analytical formulae in (7) as the Cauchy residues. We perform the characteristic polynomial rooting by expediently solving (with machine accuracy) the equivalent eigenvalue problem of the extremely sparse Hessenberg, or companion matrix [67].

The complex-valued total shape spectra in the ersatz and usual modes are provided through (13) and (14) via the Heaviside partial fractions:

$$
\begin{aligned}
& \left(\frac{P_{K}^{+}(z)}{Q_{K}^{+}(z)}\right)^{\mathrm{E}} \equiv \sum_{k=1}^{K}\left(\frac{P_{K}^{+}(z)}{Q_{K}^{+}(z)}\right)_{k}^{\mathrm{E}}=\sum_{k=1}^{K} \frac{\left|d_{k}^{+}\right| z}{z-z_{k, Q}^{+}} \quad \text { (Ersatz envelope) } \\
& \left(\frac{P_{K}^{+}(z)}{Q_{K}^{+}(z)}\right)^{\mathrm{U}} \equiv \sum_{k=1}^{K}\left(\frac{P_{K}^{+}(z)}{Q_{K}^{+}(z)}\right)_{k}^{\mathrm{U}}=\sum_{k=1}^{K} \frac{d_{k}^{+} z}{z-z_{k, Q}^{+}} \quad \text { (Usual envelope) }
\end{aligned}
$$


respectively. The difference between the lhs of (14) and (19) for the $k$ th usual component $\left(P_{K}^{+} / Q_{K}^{+}\right)_{k}^{\mathrm{U}}$ and the usual envelope $\left(P_{K}^{+} / Q_{K}^{+}\right)^{\mathrm{U}}$, respectively, is the omitted subscript $k$ in the latter and, by the same token, for the ersatz modes in (13) and (18).

As mentioned, because of the lack of interference between the absorption and dispersion modes, the ersatz component spectra are helpful for visualizing the overlap of closely lying or hidden resonances. However, there are caveats about which one must be aware in interpreting these ersatz spectra. Namely, ersatz peak heights do not reflect the actual abundance of the metabolites. Rather, usage of the actual numerical reconstructed parameters, including the phases $\varphi_{k}^{+} \neq 0$, is essential to ascertain the true abundance of metabolites, whenever the phases $\left\{\varphi_{k}^{+}\right\}$are retrieved as non-zero quantities from the encoded MRS time signal. Although the number of component resonances $\left(P_{K}^{+} / Q_{K}^{+}\right)_{k}^{\mathrm{U}}$ and $\left(P_{K}^{+} / Q_{K}^{+}\right)_{k}^{\mathrm{E}}$ is the same, their full widths at half maxima (FWHM) are not equal. Consequently, the peak areas of a given $k$ th component will differ in the usual and ersatz modes. Overall, the parameters $\left\{\omega_{k, Q}^{+}, d_{k}^{+}\right\}$with $\varphi_{k}^{+} \neq 0$ from the usual components $\left(P_{K}^{+} / Q_{K}^{+}\right)_{k}^{\mathrm{U}}$ should be used in assessing metabolite concentrations, since interference effects occur for $\varphi_{k}^{+} \neq 0$. The peak areas are affected by $\varphi_{k}^{+} \neq 0$ and so are the metabolite concentrations. Overall, the usual components $\left(P_{K}^{+} / Q_{K}^{+}\right)_{k}^{\mathrm{U}}$ alongside $\left\{\omega_{k, Q}^{+}, d_{k}^{+}\right\}$should be used to extract the metabolite concentrations, rather than their ersatz counterparts $\left(P_{K}^{+} / Q_{K}^{+}\right)_{k}^{\mathrm{E}}$ with $\left\{\omega_{k, Q}^{+},\left|d_{k}^{+}\right|\right\}$.

The derivation of the envelopes in the representation of the Heaviside partial fractions (18) and (19) employs the expression $\sum_{n=0}^{\infty}\left(z_{k, Q}^{+} / z\right)^{n}=z /\left(z-z_{k, Q}^{+}\right)$ where $\left|z_{k, Q}^{+} / z\right|<1$. The implicit assumption therein is that the total length of time signal $\left\{c_{n}\right\}$ is infinite $(N=\infty)$. In reality, however, time signals are finite $(N<\infty)$. Consequently, the latter series should be truncated at $n=N-1$, and thus $\sum_{n=0}^{N-1}\left(z_{k, Q}^{+} / z\right)^{n}=\left[1-\left(z_{k, Q}^{+} / z\right)^{N}\right] /\left(1-z_{k, Q}^{+} / z\right)$. As such, the peak heights from (15) need a correction for the factor $1-\left(z_{k, Q}^{+} / z\right)^{N}$ taken at sweep frequency $v$ which matches $\operatorname{Re}\left(v_{k, Q}^{+}\right)$, i.e. at $v=\operatorname{Re}\left(v_{k, Q}^{+}\right)$. The corrected peak heights should, therefore, read as:

$$
\begin{aligned}
\operatorname{Re}\left(H_{k}^{+}\right)^{\mathrm{U}} & \equiv \frac{\left|d_{k, T}^{+}\right| \cos \left(\varphi_{k}^{+}\right)}{D_{k}^{+}},\left(H_{k}^{+}\right)^{\mathrm{E}} \equiv \frac{\left|d_{k, T}^{+}\right|}{D_{k}^{+}}, \\
\left|d_{k, T}^{+}\right| & =\left|d_{k}^{+}\right|\left\{1-\exp \left(-T / T_{2 k}^{*+}\right)\right\}, T=N \tau,
\end{aligned}
$$

where $0<1-\exp \left(-T / T_{2 k}^{*+}\right)<1$.

In the $\mathrm{FPT}^{(+)}$, the "Stability test" involves computing consecutive values of spectra of partial signal lengths $N_{\mathrm{P}}\left(N_{\mathrm{P}}=2 K\right.$ for $N_{\mathrm{P}}$ even $)$ for components and total shape spectra:

$$
\begin{aligned}
& \left(\frac{P_{K+m}^{+}(z)}{Q_{K+m}^{+}(z)}\right)_{k}^{\mathrm{U}}=\left(\frac{P_{K}^{+}(z)}{Q_{K}^{+}(z)}\right)_{k}^{\mathrm{U}} \quad(m=1,2, \ldots), \\
& \left(\frac{P_{K+m}^{+}(z)}{Q_{K+m}^{+}(z)}\right)^{\mathrm{U}}=\left(\frac{P_{K}^{+}(z)}{Q_{K}^{+}(z)}\right)^{\mathrm{U}} \quad(m=1,2, \ldots),
\end{aligned}
$$


respectively. Only when convergence has been attained, as $N_{\mathrm{P}}$ is systematically increased are the fundamental frequencies and amplitudes $\left\{\omega_{k, Q}^{+}, d_{k}^{+}\right\}$acceptable. Total shape spectra from (22) can be computed non-parametrically or parametrically. By evaluating $P_{K}^{+}(z) / Q_{K}^{+}(z)$ for $z=\exp (2 \pi i v \tau)$ at any chosen set of real-valued sweep frequencies $v$, the non-parametric spectrum is obtained. For the parametric case, the total cross sections via the Heaviside partial fraction decompositions (18) and (19) are employed in terms of the ersatz and usual components, respectively.

\section{Results}

\subsection{The in vivo MRS time signal encoded from a borderline serous cystic ovarian lesion}

The MRS time signal encoded from a BL serous cystic ovarian lesion with a total number of 1024 data points is shown in the top two panels of Fig. 1. As noted, the present paper uses the FID encoded with a TE of $30 \mathrm{~ms}$. The real part of the encoded time signal is displayed on the top left panel (a), with the imaginary part on the top right panel (d) of Fig. 1. Across the abscissae of panels (a) and (d) is a magenta line, from which it is seen that at about $300 \mathrm{~ms}$, the FID exhibits oscillations nearly symmetrically around the abscissa. However, below $300 \mathrm{~ms}$ the FID waveforms are asymmetric around the abscissae because the residual water peak is much more abundant (about 7 times) compared to all the other metabolites.

\subsection{Total shape spectra reconstructed by the DFT and FPT $^{(-)}$}

The middle panels (b) and (e) of Fig. 1 display the total shape spectra reconstructed by the DFT and the non-parametric $\mathrm{FPT}^{(-)}$for the full Nyquist range from about -0.2 to $9.7 \mathrm{ppm}$, at a partial signal length, $N_{\mathrm{P}}=800(K=400)$. The dominant resonance in the spectra reconstructed by the DFT and $\mathrm{FPT}^{(-)}$is seen at $4.5 \mathrm{ppm}$. This represents the water residual resonance which is in the spectral dispersion mode. The other resonances, although much smaller than the residual water peak, can still be seen across the Nyquist range particularly around $2.0 \mathrm{ppm}$. At first glance, the spectra for the entire Nyquist interval appear quite similar in the reconstructions by the DFT and $\mathrm{FPT}^{(-)}$.

At the same partial signal length $N_{\mathrm{P}}=800$, the bottom panels (c) and (f) of Fig. 1 show the total shape spectra reconstructed by the DFT and the non-parametric FPT $^{(-)}$between $\sim 1.2$ and $3.4 \mathrm{ppm}$. Since the upper bound of the chemical shift region excludes the resonant frequency of water at $4.5 \mathrm{ppm}$, the other metabolites which appeared very small in panels (b) and (e), are now well visualized in panels (c) and (f). The dominant peak in both total shape spectra is centered at about $2.06 \mathrm{ppm}$. Its amplitude is slightly lower in the DFT compared to the $\mathrm{FPT}^{(-)}$and the serration to the right is more distinct in the $\mathrm{FPT}^{(-)}$. The multiply serrated structure centered around $1.35 \mathrm{ppm}$ is also somewhat blunted in the DFT. In particular, the serration at about $1.3 \mathrm{ppm}$, corresponding to threonine, Thr, is notably absent in the DFT. In the $\mathrm{FPT}^{(-)}$there are two peaks deeply split apart, corresponding to glutamine (Glu) 
In Vivo MRS for Ovarian Tumor: Non-Parametric Signal Processing; Fourier, DFT Versus Pade, FPT ${ }^{(-)}$ Using FID Data Encoded from a Patient at $B_{0}=3 \mathrm{~T}, \mathrm{TE}=30 \mathrm{~ms}, \mathrm{BW}=1200 \mathrm{~Hz}, \mathrm{TR}=2 \mathrm{~s}, \mathrm{NEX}=64$

(a)

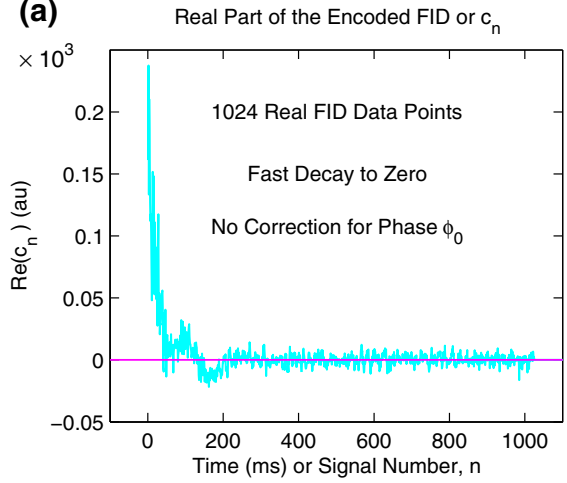

(b)

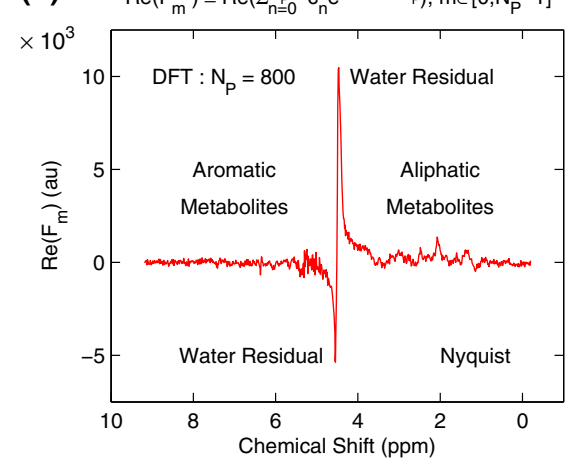

(c) $\quad \operatorname{Re}\left(F_{m}\right) \equiv \operatorname{Re}\left(\Sigma_{n=0}^{N_{p}-1} c_{n} e^{-2 \pi i n m / N}\right), m \in\left[0, N_{p}-1\right]$

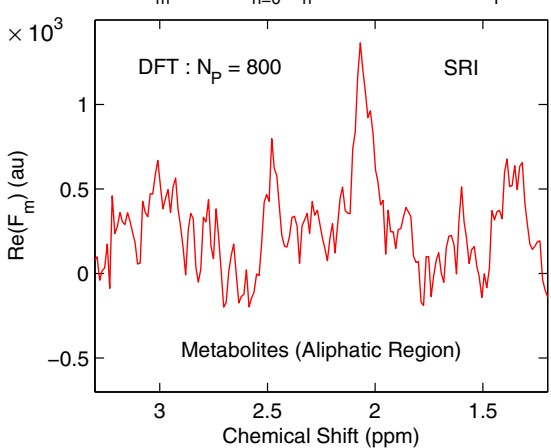

(d) Imaginary Part of the Encoded FID or $\mathrm{c}_{\mathrm{n}}$

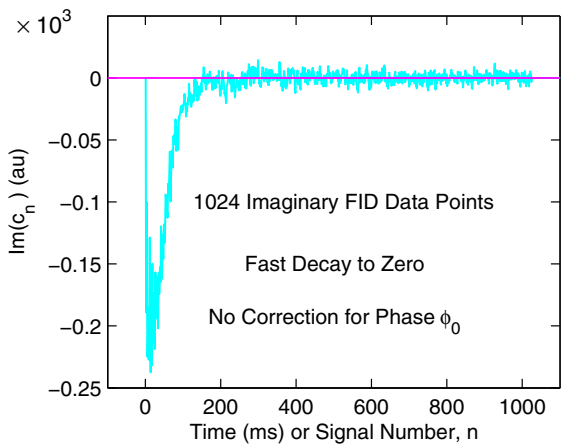

(e)
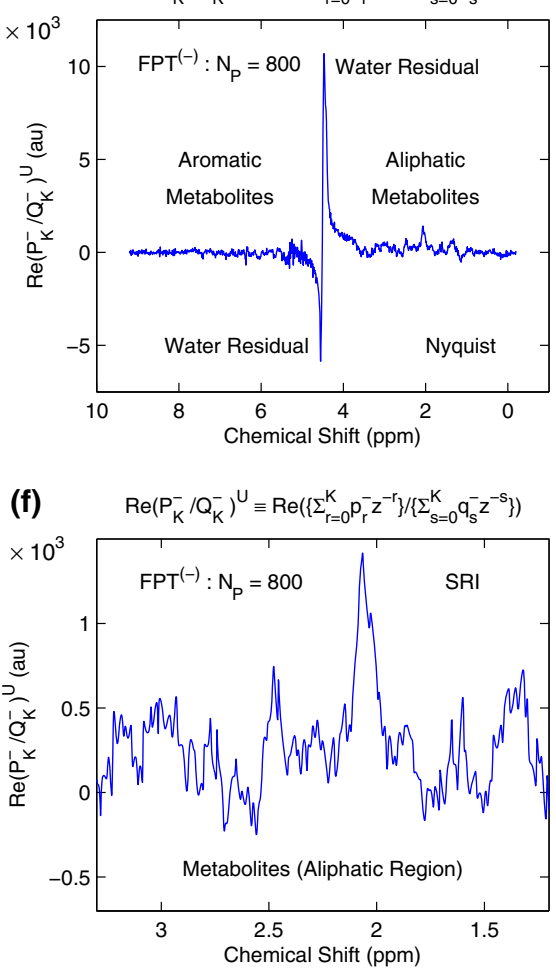

Fig. 1 The real (a) and imaginary (d) parts of the FID encoded in vivo with a $3 \mathrm{~T}$ MR scanner from a borderline, BL, serous cystic ovarian lesion. Water partially suppressed via the WET procedure in the course of encoding. Horizontal magenta lines guide the eye through departures from the level of the zero-valued amplitudes in the oscillations of the FID. Encoded FID data courtesy of the group from Ref. [50]. The real parts of the total shape spectra for the encoded FID for the full Nyquist range reconstructed by the DFT (b) and non-parametric $\mathrm{FPT}^{(-)}(\mathbf{e})$. The large resonance at $4.5 \mathrm{ppm}$ represents residual water. The real parts of the total shape spectra for the chemical shift window or SRI between $\sim 1.2$ and $3.4 \mathrm{ppm}$, as generated by the DFT (c) and non-parametric $\mathrm{FPT}^{(-)}(\mathbf{f})$ (Color online) 
plus Gln, at about $2.45 \mathrm{ppm}$. However, here at $2.45 \mathrm{ppm}$ the DFT shows only one peak with a slight shoulder to the right. Overall, at this relatively short partial signal length, with the DFT the resolution of the total shape spectrum between $\sim 1.2$ and $3.4 \mathrm{ppm}$, i.e. well below the large residual water resonance, is substantially poorer compared to the $\mathrm{FPT}^{(-)}$. A number of the peaks in the DFT are blunter and coarser, while several additional structures can be seen in the $\mathrm{FPT}^{(-)}$. From now on, we shall analyze only the results for the $\mathrm{FPT}^{(+)}$for which all the envelopes will be computed non-parametrically.

\subsection{Averaging of envelopes through the FPT $^{(+)}$}

\subsubsection{For the full Nyquist range}

In Fig. 2a, the real parts of 11 usual envelopes $\operatorname{Re}\left(P_{K}^{+} / Q_{K}^{+}\right)^{\mathrm{U}}$ generated by the nonparametric $\mathrm{FPT}^{(+)}$using the encoded FID are shown for $K=575,580, \ldots, 625$, with an increment of 5. This is for the full Nyquist range between -0.2 and $9.7 \mathrm{ppm}$. Note that the green color is used in panel (a). Besides the largest structure of the residual water peak, there are many large noise-like spikes, especially in the region above $4.8 \mathrm{ppm}$. The largest of these is around $6.5 \mathrm{ppm}$, and there are several prominent spikes between $\sim 4.9$ and $5.4 \mathrm{ppm}$. In the chemical shift region below $4.5 \mathrm{ppm}$ where the residual water peak resides, the tallest spike is at about $3.5 \mathrm{ppm}$. In panel (b) of Fig. 2, we see the result of the arithmetic average of these 11 envelopes as denoted by $\operatorname{Re}\left\{\mathrm{FPT}^{(+)}\right\}_{\mathrm{Av}}^{\mathrm{U}}$. Therein, a "clean" spectrum, is produced. This is marked in blue. The stable structures remain in this average spectrum, whereas the spikes are greatly diminished or have even disappeared in some places. In panel (c) the results of panels (a) and (b) are superimposed with the colors retained, namely the real parts of the 11 usual envelopes are presented in green, while their average envelope is in blue. Therein, it can most clearly be seen that there is a marked attenuation of all the spikes in the average envelope. Notably, even in the presence of the giant residual water peak, the distinctly split NAA and acNeu peaks in the chemical shift range 2.0-2.1 ppm can be identified.

\subsubsection{Spectral region of interest (SRI)}

For this and all subsequent figures, our focus will be upon a spectral range of interest, SRI, chosen between 0.75 and $3.75 \mathrm{ppm}$. With this selected SRI, the giant residual water peak and its surroundings are formally not in the field of view. However, as mentioned earlier, the residual water peak has not presently been suppressed and, thus, it contributes to all the computed total shape spectra.

We now proceed in Fig. 3 in a similar fashion as in the previous figure, but within the said SRI. In the top panel (a), again in green, we show the real parts of 11 usual envelopes $\operatorname{Re}\left(P_{K}^{+} / Q_{K}^{+}\right)^{\mathrm{U}}$ generated by the non-parametric $\mathrm{FPT}^{(+)}$for $K=575,580, \ldots, 625\left(N_{\mathrm{P}}=1150,1160, \ldots, 1250\right)$, with an increment of 5 ; the largest structure is a spike at about $3.4 \mathrm{ppm}$. There are numerous other tall spikes 
In Vivo MRS for Ovarian Tumor: Spectra Averaging in $\mathrm{FPT}^{(+)}$in the Full Nyquist Range Use of Encoded Time Signal Data in 11 Envelopes to Generate Their Arithmetic Average

(a) Real Parts of 11 Usual Envelopes: $\operatorname{Re}\left(P_{K}^{+} / Q_{K}^{+}\right)^{U} \equiv \operatorname{Re}\left(\sum_{r=1}^{K}\left\{p_{r}^{+} z^{r}\right\} /\left\{\Sigma_{s=0}^{K} q_{s}^{+} z^{s}\right\}\right), K \in[575,625]\{$ Incr:5\}

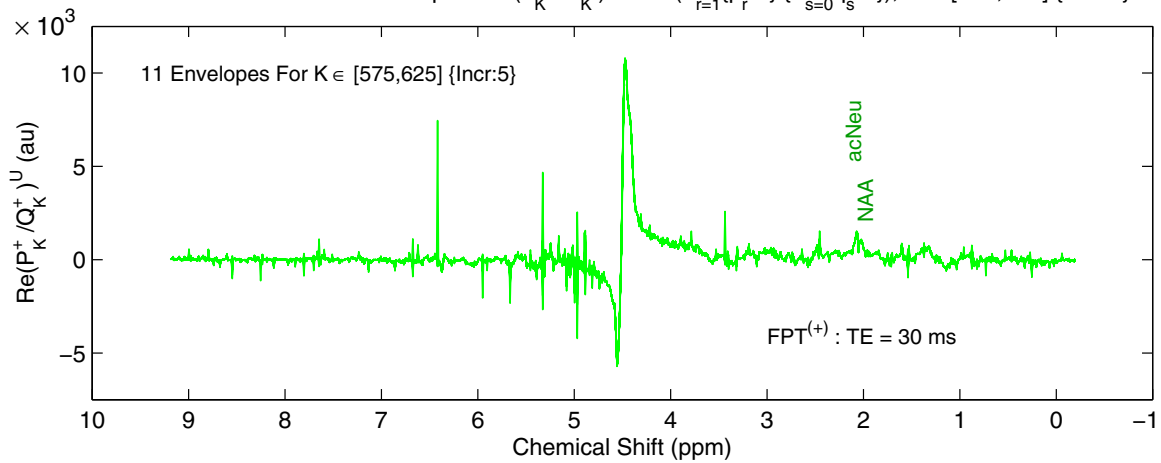

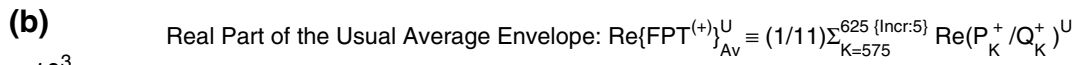

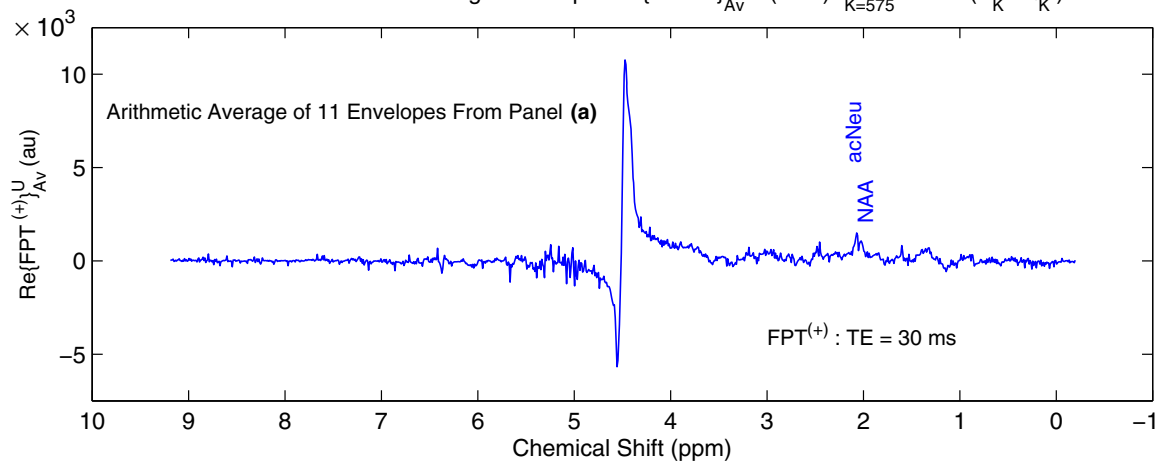

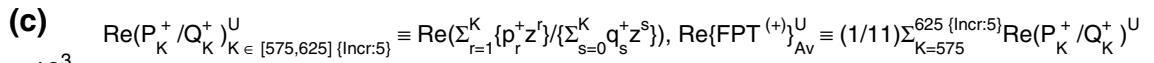

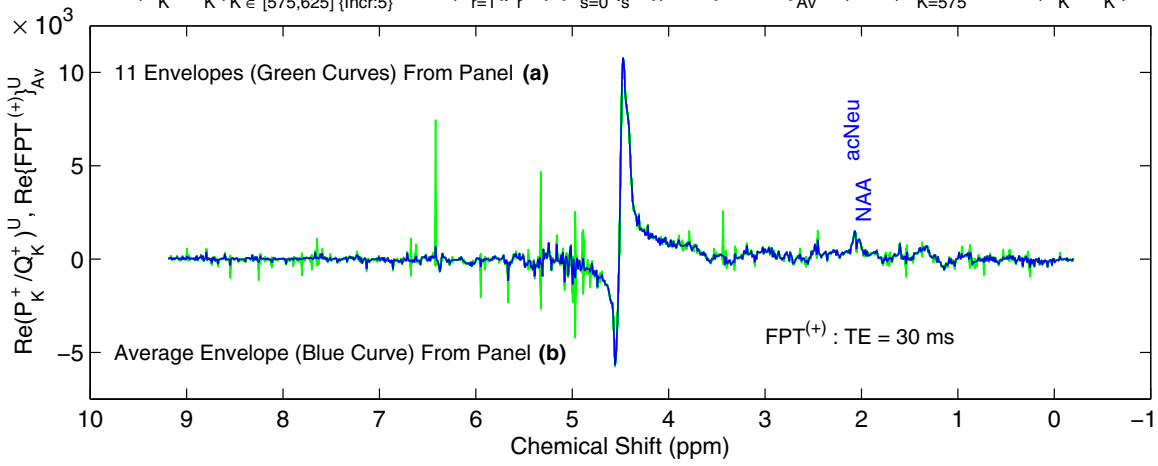

Fig. 2 The real parts of 11 usual envelopes $\operatorname{Re}\left(P_{K}^{+} / Q_{K}^{+}\right)^{\mathrm{U}}$ for $K=575,580, \ldots, 625$, with increment $\Delta K=5$, from the FID encoded in vivo from a BL serous cystic ovarian lesion [50], using a $3 \mathrm{~T}$ MR scanner for the full Nyquist range (a). Many large noise-like spikes are seen. These 11 envelopes are averaged as denoted by $\left\{\mathrm{FPT}^{(+)}\right\}_{\mathrm{Av}}^{\mathrm{U}}(\mathbf{b})$, where a "clean" spectrum is generated. The plots from (a) and (b) are superimposed in (c) (Color online) 
In Vivo MRS for Ovarian Tumor : Spectra Averaging in $\mathrm{FPT}^{(+)}$Within SRI, $v_{\mathrm{k}, \mathrm{Q}}^{+} \in[0.75,3.75] \mathrm{ppm}$

(a)

Use of Encoded Time Signal Data in 11 Envelopes to Generate Their Arithmetic Average

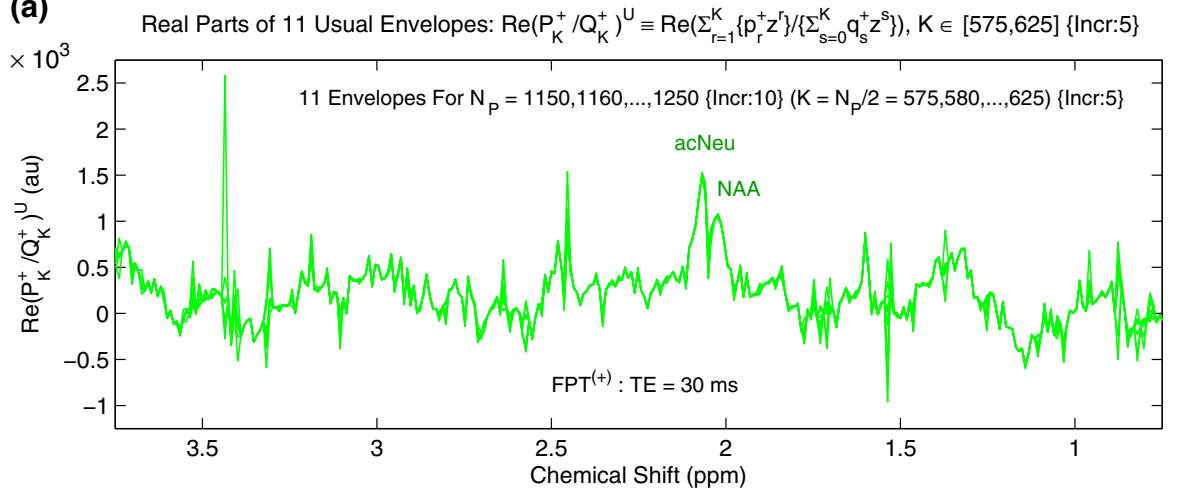

(b) Real Part of the Usual Average Envelope: $\operatorname{Re}\left\{\mathrm{FPT}^{(+)}\right\}_{\mathrm{Av}}^{\mathrm{U}} \equiv(1 / 11) \Sigma_{\mathrm{K}=575}^{625}\left\{\operatorname{Rinc:5\} } \mathrm{Re}\left(\mathrm{P}_{\mathrm{K}}^{+} / \mathrm{Q}_{\mathrm{K}}^{+}\right)^{U}\right.$

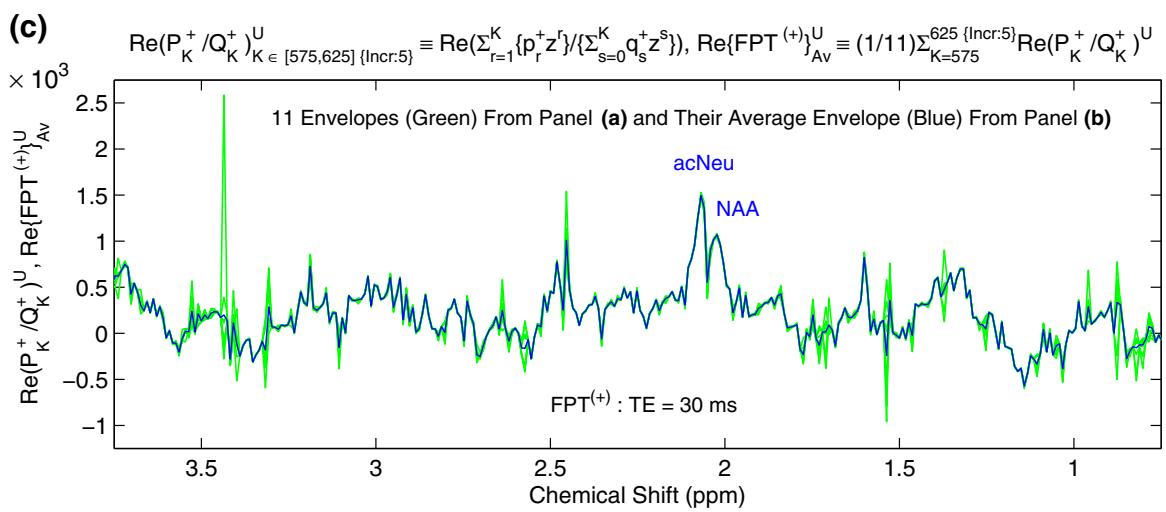

Fig. 3 The real parts of 11 usual envelopes $\operatorname{Re}\left(P_{K}^{+} / Q_{K}^{+}\right)^{\mathrm{U}}$ for $K=575,580, \ldots, 625$, with increment $\Delta K=5$, with the FID encoded in vivo from a BL serous cystic ovarian lesion [50], with a $3 \mathrm{~T}$ MR scanner in the SRI $=[0.75,3.75] \mathrm{ppm}(\mathbf{a})$. Many large noise-like spikes are seen. These 11 envelopes are averaged as denoted by $\left\{\mathrm{FPT}^{(+)}\right\}_{\mathrm{Av}}^{\mathrm{U}}(\mathbf{b})$, where a "clean" spectrum is generated. Metabolite assignments are shown in (b). See the list of abbreviations for full names. Plots from (a) and (b) are superimposed in (c) (Color online) 
interspersed throughout the entire SRI. Nevertheless, the split between NAA and acNeu peaks at about $2.05 \mathrm{ppm}$ is still very prominent.

The arithmetic average of these 11 envelopes denoted by $\operatorname{Re}\left\{\mathrm{FPT}^{(+)}\right\}_{\mathrm{Av}}^{\mathrm{U}}$ within the SRI is shown in panel (b). As in Fig. 2b, this average envelope is also marked in blue. The abbreviations for the metabolites are written above the corresponding peaks. Overall, the two largest structures are genuine resonances, centered at about $2.05 \mathrm{ppm}$. These two peaks appear with a deep split between them, clearly delineating the taller narrower acNeu $(2.06 \mathrm{ppm})$ resonance from the shorter and wider NAA (2.03 ppm). Abundant other structures are also identified as marked on panel (b). These assignments are based upon Refs. [50,59,61,62]. Starting from about 0.94 ppm, a doublet and triplet of Leu can be discerned, followed by Iso, Val and glycine (Gly) at $\sim 1.02,1.04$ and $1.20 \mathrm{ppm}$, respectively. A Lip resonance, followed by a Thr peak, a second resonance of Lip and a Lac doublet are seen on a protuberance centered at about $1.3 \mathrm{ppm}$. In the valley thereafter, a multiplet of Iso centered at 1.48 ppm is seen and then a Lys multiplet with a prominent peak around $1.52 \mathrm{ppm}$. An Ala doublet is noted thereafter, with one of the peaks being quite tall. Within the subsequent valley, two small Leu peaks are seen centered at about $1.73 \mathrm{ppm}$, and then a Lys peak. Within the next protuberance are a small Ace doublet and then a Lys doublet, abutting on the right side of the large NAA peak. A number of small peaks: Gln, Met, Gly and Pyr are seen to the left of AcNeu. Then, the large Glu and Gln peaks appear centered at about $2.45 \mathrm{ppm}$. Abutting on the left side is a small Cit peak, followed by a myoinositol ( $\mathrm{m}$-Ins) triplet centered at $\sim 2.6 \mathrm{ppm}$, which is followed by betaine (Bet), Gln, NAA and then Cit. At $3.0 \mathrm{ppm}$ a small $\mathrm{Cr}$ peak can be seen and to its left is phosphocreatine ( $\mathrm{PCr}$ ), followed by a small tyrosine (Tyr) multiplet and a creatinine (Crn) peak. At $3.2 \mathrm{ppm}$, a narrow, distinct Cho resonance can be seen, adjacent to which is a smaller PC resonance at $\sim 3.22 \mathrm{ppm}$, followed by a small glycerophosphocholine (GPC) peak. Another Bet peak is then seen at about $3.27 \mathrm{ppm}$, followed by histidine (His), mannose (Mann) and a multiplet of m-Ins. At $\sim 3.54 \mathrm{ppm}$ is a miniscule glucose (Glc) peak after which is another m-Ins multiplet and Gly at the upper extent of the SRI.

The results of panels (a) and (b), with the retained green and blue colors, respectively, are superimposed in panel (c) of Fig. 3. This provides a striking contrast between the spurious structures that, in many cases, are much taller than the genuine peaks. Most dramatically, the spike at about 3.4 ppm completely overwhelms the tiny peaks in that chemical shift region. The Glu peak at about $2.45 \mathrm{ppm}$ is entirely overridden by the larger spike. On a smaller scale, the Lac doublet centered at about $1.34 \mathrm{ppm}$ is substantially more difficult to discern in the presence of the spike in that region. Panel (c) clearly demonstrates the value of spectral averaging in reducing the spurious content so that the genuine structures, which are very small in many instances, can be visualized even on the total shape spectrum.

Of particular importance is to note that the 12 spectra (11 in green and 1 in blue) are practically coincident in e.g. the extended interval $1.8-2.45 \mathrm{ppm}$ with no spikes at all. Here, dense genuine resonances abound with the dominance of acNeu and NAA. In fact, therein, all the physical resonances are so tightly packed together that virtually no spurious structure can enter this interval. Overall, close agreement among the 11 green curves and their arithmetic average plotted in blue at $v \in[1.8,2.45] \mathrm{ppm}$ as seen 
in panel (c) of Fig. 3 permits the following conclusion to be drawn. For a high density of genuine metabolites condensed in an extended band of the given SRI, convergence is manifestly accurate and fast, thus making obsolete the over-sensitivity of Padéestimation to model order $K$. It then appears that the so-called "spectral crowding" (abundance of closely located physical peaks, especially for FIDs encoded at short TEs) is actually beneficial for the FPT. The reason for this is that the Padé estimation is most accurate for detecting the genuine resonances present in the processed MRS data. This is dictated by e.g. the polar representation of the parametric Padé spectrum by which every true signal pole in the analyzed FID will straightforwardly be reconstructed with utmost precision and efficiency. The same feature is also implicitly present in the non-parametrically computed Padé spectrum $P_{K} / Q_{K}$ since the polar singularities are sensed by the minimal values of the denominator polynomial $Q_{K}$.

These remarks highlight the default advantage of rational (Padé) over ordinary (Fourier) polynomials for signal processing in MRS and beyond. Functions with polar singularities, such as those in all frequency spectra from MRS are most naturally described by ratios of two polynomials $P_{K} / Q_{K}$ since the spectral zeros and poles are automatically and expediently provided by the roots of the numerator $\left(P_{K}\right)$ and denominator $\left(Q_{K}\right)$ polynomials, respectively. By contrast, for the same spectra built from polar functions, Fourier processing with a single polynomial $F_{m}(0 \leq m \leq N-1)$ from (1a) is notoriously inadequate. This occurs because all polynomials are nonsingular, implying that a very long signal length $N$ (corresponding to a long acquisition time $T$ ) is necessary with abundant constructive and destructive interferences required to generate all the peaks located near the actual signal poles.

\subsection{Reconstruction of the component spectra through the $\mathrm{FPT}^{(+)}$}

We now proceed in Fig. 4 to the parametric analysis through the $\mathrm{FPT}^{(+)}$by which the actual components of envelopes can be visualized. It should be particularly noted that the chemical shifts along the abscissae of panels (a)-(c) of Fig. 4 are precisely aligned, so that the reader can easily examine these three panels in concert.

The average envelope from Fig. 3b, including all the metabolite assignments, is repeated in Fig. 4a. The complex-valued counterpart of this average envelope was subsequently inverted by the IDFT to generate an FID to which the parametric $\mathrm{FPT}^{(+)}$ was applied. The partial signal length used was $N_{\mathrm{P}}=1200$, i.e. $K=600$, which is in the middle of the interval $K=575$ to 625 , from which the 11 envelopes from Fig. 3 a were reconstructed.

Panel (b) displays the usual component spectra. Therein, a very dense admixture is seen of absorption and dispersion spectra, due to the amplitudes $\left\{d_{k}^{+}\right\}$being complexvalued with non-zero phases $\varphi_{k}^{+} \neq 0(1 \leq k \leq K)$. Nevertheless, much diagnostic information can be gleaned with careful inspection. It is notable that the NAA peak appears to be larger than acNeu. The reason for this is that the acNeu is in the dispersion mode, with a narrow positive lobe as well as a sizable negative lobe. This latter negative lobe is responsible for lowering the peak height of acNeu relative to the case when acNeu would be in a pure absorptive mode. In contrast, NAA is mainly in the absorption mode, appearing as a slightly skewed absorptive Lorentzian. In the chemical shift 
In Vivo MRS for Ovarian Tumor: Average Envelope and Component Spectra (Usual, Ersatz) in FPT ${ }^{(+)}$ Component Spectra Stem From the Time Signal Given by the Inverted Complex Average Envelope

(a) Real Part of the Usual Average Envelope: $\operatorname{Re}\left\{\mathrm{FPT}^{(+)}\right\}_{\mathrm{Av}}^{U} \equiv(1 / 11) \Sigma_{\mathrm{K}=575}^{625\{\mathrm{Incr:5 \}}} \operatorname{Re}\left(\mathrm{P}_{\mathrm{K}}^{+} / \mathrm{Q}_{\mathrm{K}}^{+}\right)^{\mathrm{U}}$

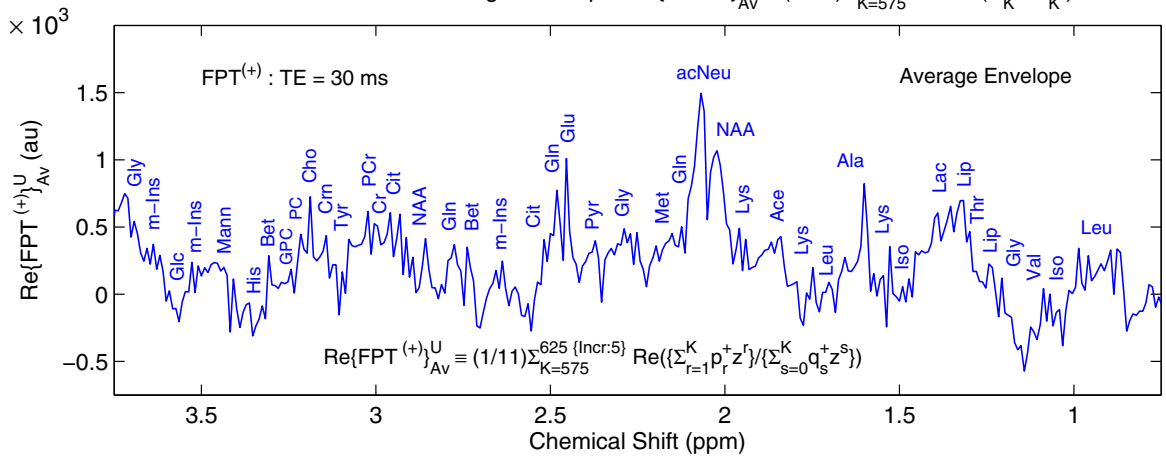

(b) Real Parts of the Usual Component Shape Spectra: $\left.\operatorname{Re}\left(P_{K}^{+} / Q_{K}^{+}\right)_{k}^{U} \equiv d_{k}^{+} z /\left(z-z_{k, Q}^{+}\right)\right),\left\{Q_{K}^{+}\left(z_{k, Q}^{+}\right)=0\right\}$

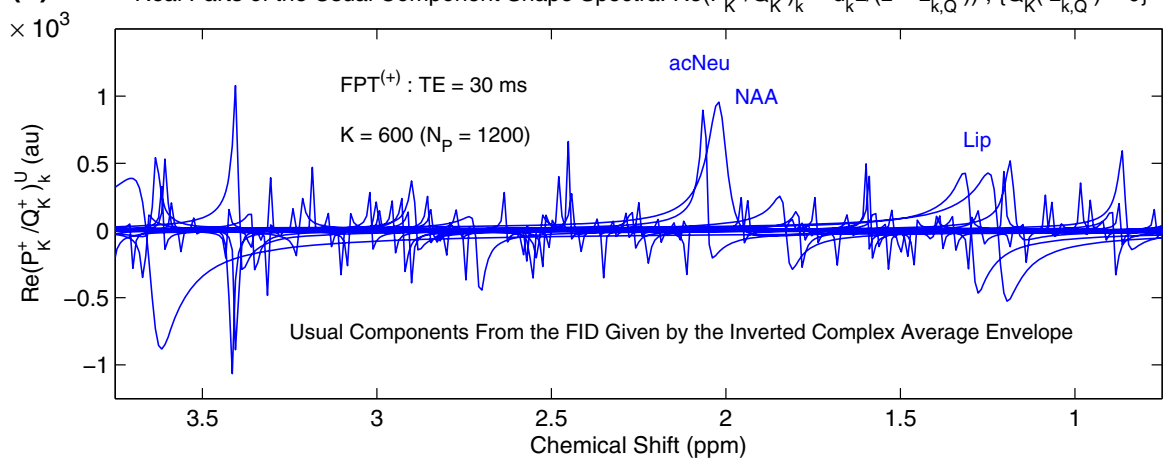

(c) Real Parts of the Ersatz Component Shape Spectra: $\left.\operatorname{Re}\left(P_{K}^{+} / Q_{K}^{+}\right)_{k}^{E} \equiv d_{k}^{+} \mid z /\left(z-z_{k, Q}^{+}\right)\right),\left\{Q_{K}^{+}\left(z_{k, Q}^{+}\right)=0\right\}$

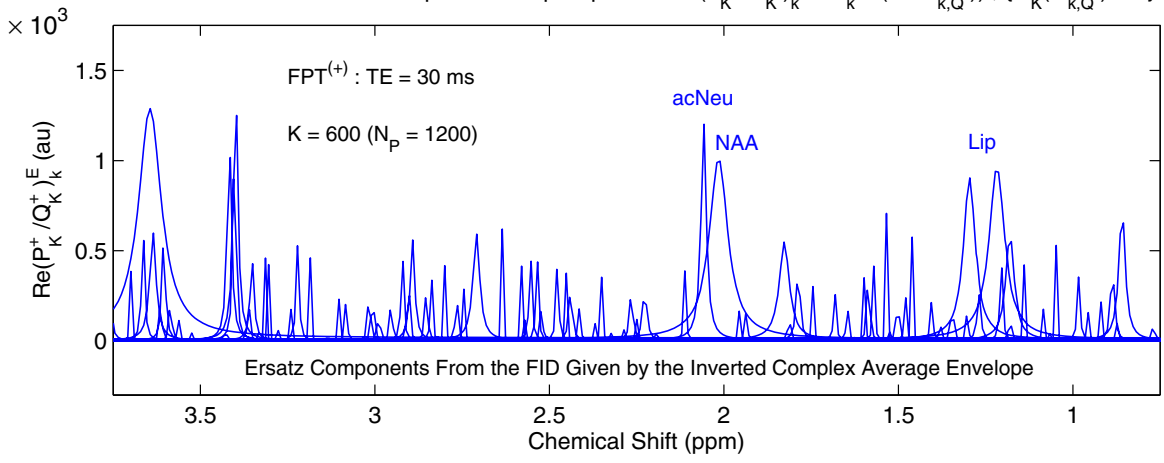

Fig. 4 The FID from the IDFT-based inversion of the complex average envelope, with the real part shown in (a) is subjected to the $\mathrm{FPT}^{(+)}$to generate the usual and ersatz component spectra in (b) and (c), respectively. The usual components (b) mix the absorption and dispersion lineshapes. Ersatz component spectra (c) display many closely-overlapping positively-oriented resonances, all in the absorption mode (Color online) 
region around $1.3 \mathrm{ppm}$, two resonances in the dispersion mode are the most prominent. These correspond to lipids responsible for the protuberance on the total shape spectrum in that region. Namely, as noted, they can also be seen on the total shape spectrum as isolated Lip peaks at about 1.25 and $1.34 \mathrm{ppm}$, with Thr in between them. The Thr and Lac resonances are relatively small in the usual component spectra and, as mentioned, appear in the total shape spectrum as serrations jutting above the protuberance, which we now can see is mainly due to Lip. Large, wide resonances are also observed in the chemical shift region from about 3.45 to $3.75 \mathrm{ppm}$. These resonances are also in the dispersion mode, with the negative lobes substantially larger than the positive lobes. These resonances correspond to macromolecules and contribute to the marked oscillations in the baseline in this chemical shift region. It should be recalled that with the parametric FPT, when the SRI excludes the chemical shift region of water, as is the case herein, there is no structure whatsoever from the residual water peak [79]. However, since the total shape spectrum, as shown in Fig. 4a, was generated via the non-parametric FPT, the residual water contributes to the rising baseline from about $3.6 \mathrm{ppm}$ to the end of the SRI.

The ersatz component spectra in panel (c) provide yet another means of distinguishing the numerous closely-overlapping resonances. Therein, interference effects are eliminated with display of purely absorptive Lorentzians with $\varphi_{k}^{+}=0(1 \leq k \leq K)$. With the removal of the phase of the amplitude, the right negative lobe of acNeu, has merged with the left positive lobe, such that acNeu peak now appears taller than NAA, as in the envelope from panel (a). Similarly, the Lip resonances centered at 1.3 ppm now appear as larger, symmetrical Lorentzians, under which the Lac and Thr doublets lie. Identification of individual resonances such as the Leu quintet towards the lower limit of the SRI, at about $0.94 \mathrm{ppm}$ is also facilitated by the ersatz component spectra. Overall, the plethora of resonances, well over 90 within this SRI, can each be distinguished. Nevertheless, despite the clarity provided by the presentation of the ersatz component spectra, the caveat should be reemphasized. Recall that in the ersatz mode, all the reconstructed phases $\varphi_{k}^{+}(1 \leq k \leq K)$ have been set to zero, "by hand". The ersatz mode cannot be used to assess the actual peak heights, since the heights of the ersatz peaks do not reflect the true abundance of the metabolites. Earlier, we made a remark concerning the chemical shifts of the ersatz peaks corresponding to the dispersive Lorentzians from the usual mode. This remark applies most clearly to the two Lip dispersion component spectra $\left(P_{K}^{+} / Q_{K}^{+}\right)_{k}^{\mathrm{U}}$ around $1.3 \mathrm{ppm}$ in panel (b) of Fig. 4. The corresponding two Lip absorptive Lorentzians $\left(P_{K}^{+} / Q_{K}^{+}\right)_{k}^{\mathrm{E}}$ from panel (c) of Fig. 4 have their peak positions $\operatorname{Re}\left(v_{k, Q}^{+}\right)$centered in between the left and right lobes of the two Lip dispersion spectra $\left(P_{K}^{+} / Q_{K}^{+}\right)_{k}^{\mathrm{U}}$ from panel (b).

\subsection{Separation of the spurious from the genuine resonances in the $\mathrm{FPT}^{(+)}$}

Figure 5 presents a detailed examination of Signal-noise separation, SNS, through the $\mathrm{FPT}^{(+)}$. Panel (a) of Fig. 5 reiterates Fig. 3c, namely the real parts of 11 usual envelopes $\operatorname{Re}\left(P_{K}^{+} / Q_{K}^{+}\right)^{\mathrm{U}}$ generated from the non-parametric $\mathrm{FPT}^{(+)}$with the numerous spurious spikes (in green) and the arithmetic average of these 11 envelopes denoted by $\operatorname{Re}\left\{\mathrm{FPT}^{(+)}\right\}_{\mathrm{Av}}^{\mathrm{U}}$ (in blue). 
In Vivo MRS for Ovarian Tumor: Reconstructed Genuine and Spurious Resonances in $\mathrm{FPT}^{(+)}$ Spurious (Noiselike) Froissart Doublets: Pole-Zero Confluences Yielding Zero-Valued Amplitudes

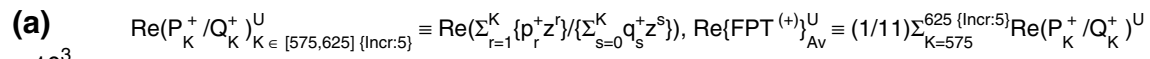
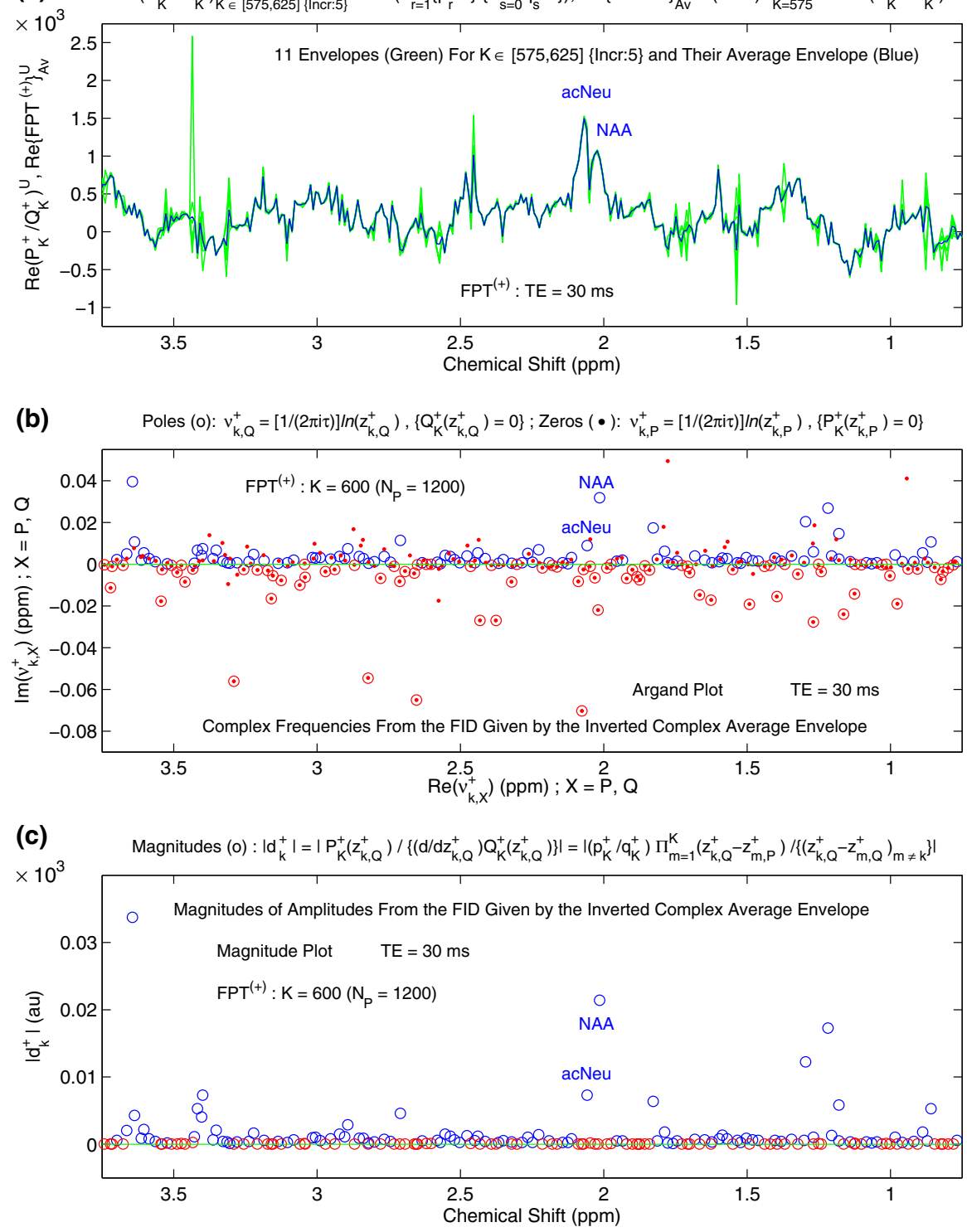

Fig. 5 The real parts of 11 usual envelopes $\operatorname{Re}\left(P_{K}^{+} / Q_{K}^{+}\right) \mathrm{U}$ for $K=575,580, \ldots, 625$, with increment $\Delta K=5$ and their average envelope (a). Signal-noise separation illustrated in (b) and (c). The Argand plot (poles: circles, zeros: dots) with complete separation of genuine from spurious frequencies (b). In the latter, pole-zero coincidences occur. The genuine and spurious poles are shown in blue and red circles, respectively. In (c), a magnitude plot $\left|d_{k}^{+}\right|$versus chemical shift shows zero-valued magnitudes of spurious resonances (red), with genuine resonances (blue) having non-zero magnitudes (Color online) 
We now proceed to the parametric analysis, for which, as in the previous subsection, the complex average envelope was inverted by the IDFT to generate an FID to which the parametric $\mathrm{FPT}^{(+)}$was applied using a partial signal length $N_{\mathrm{P}}=1200$, i.e. $K=600$. In panel (b) the Argand plot is shown, namely the Padé-reconstructed imaginary versus real frequencies, $\operatorname{Im}\left(v_{k, Q}^{+}\right)$versus $\operatorname{Re}\left(v_{k, Q}^{+}\right)$, respectively. Open circles denote poles and dots symbolize zeros. An auxiliary horizontal green line facilitates visualization of the separation between the genuine and spurious frequencies that lie in the positive and negative imaginary frequency regions, respectively. In the latter region, the poles are also depicted in red to distinctly point out the pole-zero coincidences that indicate spuriousness. Well over 60 pole-zero coincidences can be identified within the SRI, many of which are quite densely packed. It should also be noted that the negative extent of the ordinate is limited to $-0.09 \mathrm{ppm}$, and there are some pole-zero coincidences that lie even deeper and are thus not visualized herein. In the positive imaginary frequency region, the blue open circles denote poles which, in most cases, are empty, i.e. with no inscribed dots symbolizing spectral zeros. There are, however, some red-dotted zeros that are close to the centers of the blue circles for the genuine poles. This near polezero coincidence is known to occur for some genuine resonances [76]. Nevertheless, these are stable and, as such, do not represent Froissart doublets. It should be noted that the pole corresponding to NAA is deep within the imaginary part of the complex frequency plane. This indicates a wide resonance with faster decay, whereas the pole corresponding to acNeu is closer to zero, consistent with a narrower peak with a longer $T_{2 k}^{*+}$. Similarly, each of the resonances in the Lip doublet at around $1.34 \mathrm{ppm}$ is wide with their poles deep in the imaginary plane, as are the two poles corresponding to macromolecules in the region around $3.7 \mathrm{ppm}$. These also have shorter $T_{2 k}^{*+}$, and thus would be most likely to appear with the short TEs, such as in the present investigation.

A plot of magnitude $\left|d_{k}^{+}\right|$versus chemical shift is presented in panel (c) of Fig. 5. Open circles with blue color denote magnitudes $\left|d_{k}^{+}\right|$of amplitudes $d_{k}^{+}$of the Padéreconstructed genuine resonances. The spurious resonances have zero amplitudes and are depicted in red to aid their visualization. A direct correspondence between the pole-zero coincidences in panel (b) and the zero magnitudes is observed, as expected. The genuine resonances have non-zero magnitudes, although for several of these, the magnitudes are very small. This includes PC and GPC at $\sim 3.22$ and $3.23 \mathrm{ppm}$, respectively. The magnitudes of both Lip doublets at about $1.34 \mathrm{ppm}$ are large. For NAA at about $2.03 \mathrm{ppm}$, the magnitude is even larger, whereas the adjacent acNeu has a much smaller magnitude.

As stated earlier [see also (15) and (16)], it should be emphasized that peaks heights in the absorption mode of a conventional Lorentzian lineshape are given by $\left(H_{k}^{+}\right)^{\mathrm{c}}=$ $\left|d_{k}^{+}\right| /\left[\tau \operatorname{Im}\left(v_{k, Q}^{+}\right)\right]$. In panel (a), the NAA peak is shorter and broader than acNeu. On the other hand, the larger width of NAA at 2.03 ppm compared to acNeu at $2.06 \mathrm{ppm}$ was clearly seen in the Argand plot of panel (b) where $\left\{\operatorname{Im}\left(v_{k, Q}^{+}\right)\right\}_{\mathrm{NAA}}>$ $\left\{\operatorname{Im}\left(v_{k, Q}^{+}\right)\right\}_{\text {acNeu }}$. For this reason, there will be more attenuation of the magnitude of NAA than of acNeu and, consequently, $\left(H_{k}^{+}\right)_{\text {NAA }}^{\mathrm{c}}<\left(H_{k}^{+}\right)_{\text {acNeu }}^{\mathrm{c}}$. Thus, the patterns for NAA and acNeu seen in panels (a) - (c) are entirely coherent. 


\section{Discussion and conclusions}

This is the first study to apply the fast Padé transform, FPT, to in vivo MRS time signals encoded from the ovary. Overall, the multi-faceted Padé-based strategy that was previously established for processing in vivo time signals encoded from the brain, and from which very dense spectra were generated [79,90,91], is herein also validated for the ovary. The expectation that much richer metabolic information from the ovary would be gleaned through the FPT, than has heretofore been the case using conventional Fourier estimation with post-processing fits of the FFT spectra is now borne out. On the most basic level of comparing the total shape spectra generated at a relatively short partial signal length of $N_{\mathrm{P}}=800$ within the spectral region of interest, SRI, the non-parametric $\mathrm{FPT}^{(-)}$was shown to provide substantially better resolution than the discrete Fourier transform, DFT. This corroborates previous benchmarking studies on synthesized MRS time signals associated with the ovary, in which the high resolution capabilities of the FPT were shown [66,71,74-77].

The spectra averaging procedure $[79,91]$, in which the arithmetic average is taken of a pre-computed sequence of the retrieved envelopes (11 such envelopes in the present paper) is hereby further confirmed to be a simple and powerful way to stabilize the process of shape estimation in face of a marked sensitivity to alteration in model order $K$. The $\mathrm{FPT}^{(+)}$with its initial convergence region in the harmonic variable $z$ located inside the unit circle $(|z|<1)$, through analytical continuation, must in this region induce convergence into the input divergent series [70]. The large spikes seen when the 11 usual envelopes computed for $K=575,580, \ldots, 625$, with an increment of 5 are plotted reflect the difficulty of the task of performing analytical continuation by numerical means. When these 11 envelopes were averaged, however, a clean total shape spectrum resulted. This points to the random, noise-like nature of the instability of spurious resonances with change in model order $K$. A new insight provided by the present paper is that an increased increment, i.e. $\Delta K=5$ rather than $\Delta K=1$, between the two consecutive model orders $K$ of computed envelopes appears to be a more effective procedure, providing better washout of the spikes. It should also be noted that we have presently carried out the quantification for one $K$ in the middle of the interval. A similar pattern is seen for other values of $K$ within the interval and outside the interval of the averaging.

The total shape spectrum generated as the average of these 11 usual envelopes was seen to be informatiive. A large number of metabolites, including several doublet, triplet and even multiplet resonances could be discerned. Since the MRS time signal was encoded at a short echo time, TE, of $30 \mathrm{~ms}$, the total shape spectrum was very dense. This was due, in part, to the fact that many short-lived metabolites had not yet decayed. Consequently, clinical interpretation of such a dense envelope spectrum would be very difficult. How could one ascertain the relative abundance of each of these identified metabolites? How should the oscillating baseline be analyzed? Could there be even more resonances underlying these large structures? The answers to these questions would be utterly impossible to ascertain without parametric analysis, such as an unambiguous quantification in the FPT.

As previously emphasized in Refs. [66,71,73-76] and clearly seen in this work, it is vital to go beyond the total shape spectra to analyze the components. In the 
present study this is achieved through the parametric $\mathrm{FPT}^{(+)}$. Not only were all the true resonances clearly identified, but also their peak parameters were all precisely ascertained. A number of clinically important insights can be gleaned thereby. Firstly, in the region around $1.3 \mathrm{ppm}$, the usual and ersatz component spectra elucidate the overlap among Lip, Lac as well as Thr and other resonances. The lifting of the baseline in that region is seen to be due to wide Lip resonances. This clarification could help resolve the uncertainty as to whether or not the presence of Lip distinguishes benign from cancerous ovarian lesions. According to our meta-analysis (Table 1), Lip at $1.3 \mathrm{ppm}$ was more often identified in malignant lesions, but this difference was not statistically significant. On the other hand, the presence of Lac at $1.3 \mathrm{ppm}$ was a significant predictor of cancerous lesions, but there were mainly missing data regarding this metabolite. With Padé-based parametric analysis, a short TE can be employed so that both Lip and Lac could not only be identified, but also quantified in the chemical shift region around $1.3 \mathrm{ppm}$.

With the FPT, the presence of Cho at $3.2 \mathrm{ppm}$ was also clearly detected and its peak parameters reconstructed. For the first time phosphocholine, PC, as well as glycerophosphocholine, GPC, were both clearly delineated via in vivo MRS time signals encoded from the ovary. Since the histopathology of the present case was borderline, a relatively small PC peak could be expected. Given that PC is a recognized biomarker of malignant transformation $[64,65]$, with Padé optimization it now becomes possible to assess this key metabolite non-invasively, with an anticipated improvement in identifying ovarian cancer. Quantification of PC could also aid in timelier detection of ovarian cancer progression, based upon studies of ovarian tumor cell lines [64].

In the present meta-analysis, no diagnostic conclusions regarding the presence of NAA could be gleaned from Fourier-based analysis of in vivo MRS. In sharp contrast, through Padé reconstructions, the two resonances between 2.0 and $2.1 \mathrm{ppm}$ corresponding to $N$-acetyl aspartate, NAA, and $N$-acetylneuraminic acid, acNeu, were clearly distinguished, as being split apart nearly to the baseline level, even on the total shape spectra. With this distinction between the two closely-lying resonances, as well as the possibilities for their quantification through the FPT, clarification could be forthcoming as to the actual significance of NAA versus acNeu in identifying malignant as opposed to benign ovarian lesions.

Fundamental to the entire Padé-based strategy is to disentangle genuine from spurious resonances. In our previous proof-of-principle studies based on synthesized time signals similar to the corresponding encoded FIDs from cystic ovarian lesions with added noise, the particular utility of the $\mathrm{FPT}^{(+)}$was seen. This was due to the clear separation of the genuine and spurious resonances inside and outside the unit circle, respectively. In the present study, even after spectra averaging, the Froissart doublets within the SRI were of the order of abundance of the genuine resonances. Through the $\mathrm{FPT}^{(+)}$, the pole-zero coincidences were found in the negative imaginary frequency region, whereas the genuine resonances (poles) were all located in the positive imaginary frequency region. This separation is vitally important for identifying true (physical) resonances with very small magnitudes, especially in chemical shift regions of high density, as was the case for the present study.

It should, moreover, be emphasized that this Padé-based strategy would render the use of short echo times, TE, to be not only feasible, but desirable, given the rich 
spectral information to be gleaned thereby from short-lived resonances. This obviously requires the unequivocal disentangling of overlapping resonances, a task for which the parametric FPT is fully-equipped, as seen herein. In sharp contrast, only 3 of the 13 studies in the meta-analysis used a short TE of $30 \mathrm{~ms}$. This was undoubtedly due to the difficulties inherent therein with Fourier-based processing. In particular, the overlap of Lip and Lac has been problematic at short TE. Thus, the more common practice has been to use a TE of $136 \mathrm{~ms}$, so that an inverted Lac doublet appears due to J-modulation, while the lipids have already decayed. However, with the FPT, the Lac and Lip doublets can unequivocally be identified as separate structures by analyzing the component shape spectra. In fact, it is only after the components have become available by way of quantification that we can, with certainty, assign the known metabolites to the found distinct spectral structures in the total shape spectra, as was presently done in panel (b) of Fig. 3.

With the presently expounded and validated procedure of spectra averaging through the parametric FPT, it would also be possible in the future to choose an SRI containing frequencies above the chemical shift of the large residual water resonance. This chemical shift region may also yield diagnostic insights [62] that warrant further examination.

The present results are the first steps showing the effectiveness of the FPT for processing in vivo MRS time signals encoded from the ovary. From here, application of the FPT to in vivo MRS time signals encoded from various ovarian cancer types, as well as to a wide range of benign ovarian findings and to entirely normal appearing ovary is clearly warranted. The diversity of ovarian pathology, as can be seen in our meta-analysis, is an admitted challenge which will further require painstaking attention.

Solid versus cystic ovarian cancers appear to differ substantially. Our meta-analysis indicates that Cho was significantly less likely to be detected in voxels from cystic ovarian cancers than from voxels encoded from solid ovarian cancer tissue. The in vitro data of Boss et al. [59] showed that median concentration of Cho in the cancerous ovarian fluid ( $42 \mu \mathrm{M} / \mathrm{L}$ ww, where ww denotes wet weight) was actually the lowest of all the twelve examined metabolites. This suggests the need to broaden the exploration to include other metabolites besides Cho when investigating cystic ovarian lesions through MRS. Moreover, the vast majority of epithelial ovarian cancers reportedly contain cysts [97], the metabolic characteristics of which warrant further examination.

The overriding task would be to perform extensive multivariate exploration to find the metabolite patterns from MRS that best distinguish benign from borderline or unequivocally cancerous ovary. With the rich, quantitative metabolic information that can be gleaned through Padé-optimization, we anticipate that a substantial improvement will be forthcoming compared to the results thus far achieved with conventional Fourier-based processing of in vivo MRS time signals encoded from the ovary. The limitations of the latter are underscored by the meta-analysis performed herein. The overall paucity of in vivo MRS studies of the ovary speaks to the difficulty of acquiring good quality time signals from this small, moving organ, and that heretofore the diagnostic yield from in vivo MRS has been insufficient to motivate further clinical studies. 
The present results strongly motivate further applications of Padé-optimized in vivo MRS, with the main aim of contributing towards early ovarian cancer detection, and exploring the potential to more clearly identify benign ovarian lesions, for which unnecessary invasive procedures could be avoided. A major advantage of MR-based methods, as well as ultrasound, is that there is no exposure to ionizing radiation. Especially for women at increased risk of ovarian cancer, this is a vital consideration, since exposure to diagnostic medical radiation may be associated with further elevation in risk for radiation-induced ovarian cancer [98]. Radiation exposure may also occur with e.g. radiation therapy of cervical cancer. For women who are at increased risk of ovarian cancer, whether due to ionizing radiation exposure, hereditary and/or other risk factors, Padé-optimized MRS could be particularly helpful for surveillance. Of key importance is that early detection of ovarian cancer would greatly improve survival for women afflicted with this malignancy. Effective methods are needed to achieve this goal, for which Padé-optimized in vivo MRS definitely holds promise.

Acknowledgements This work was supported by The Marsha Rivkin Center for Ovarian Cancer Research, King Gustav the 5th Jubilee Fund, and FoUU through Stockholm County Council to which the authors are grateful. We would like to thank our colleagues, Professors Marinette van der Graaf, Leon Massuger, Ron Wevers, Eva Kolwijck, Udo Engelke, Arend Heerschap, Henk Blom, M'Hamed Hadfoune, and Wim Buurman from Radboud University for kindly making the in vivo encoded FIDs available to us.

\section{Compliance with ethical standards}

Conflict of interest The authors declare that they have no conflict of interest.

Open Access This article is distributed under the terms of the Creative Commons Attribution 4.0 International License (http://creativecommons.org/licenses/by/4.0/), which permits unrestricted use, distribution, and reproduction in any medium, provided you give appropriate credit to the original author(s) and the source, provide a link to the Creative Commons license, and indicate if changes were made. 


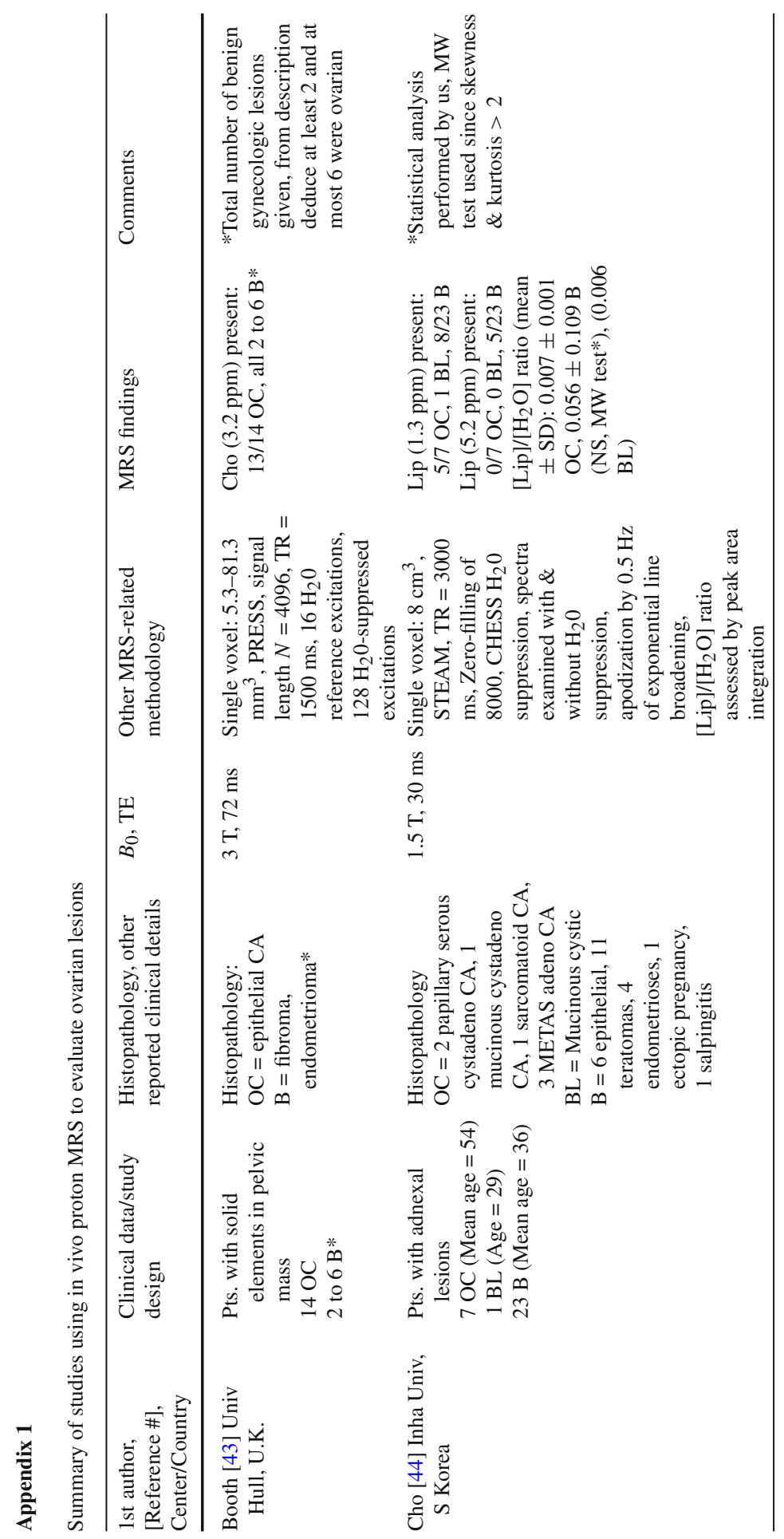




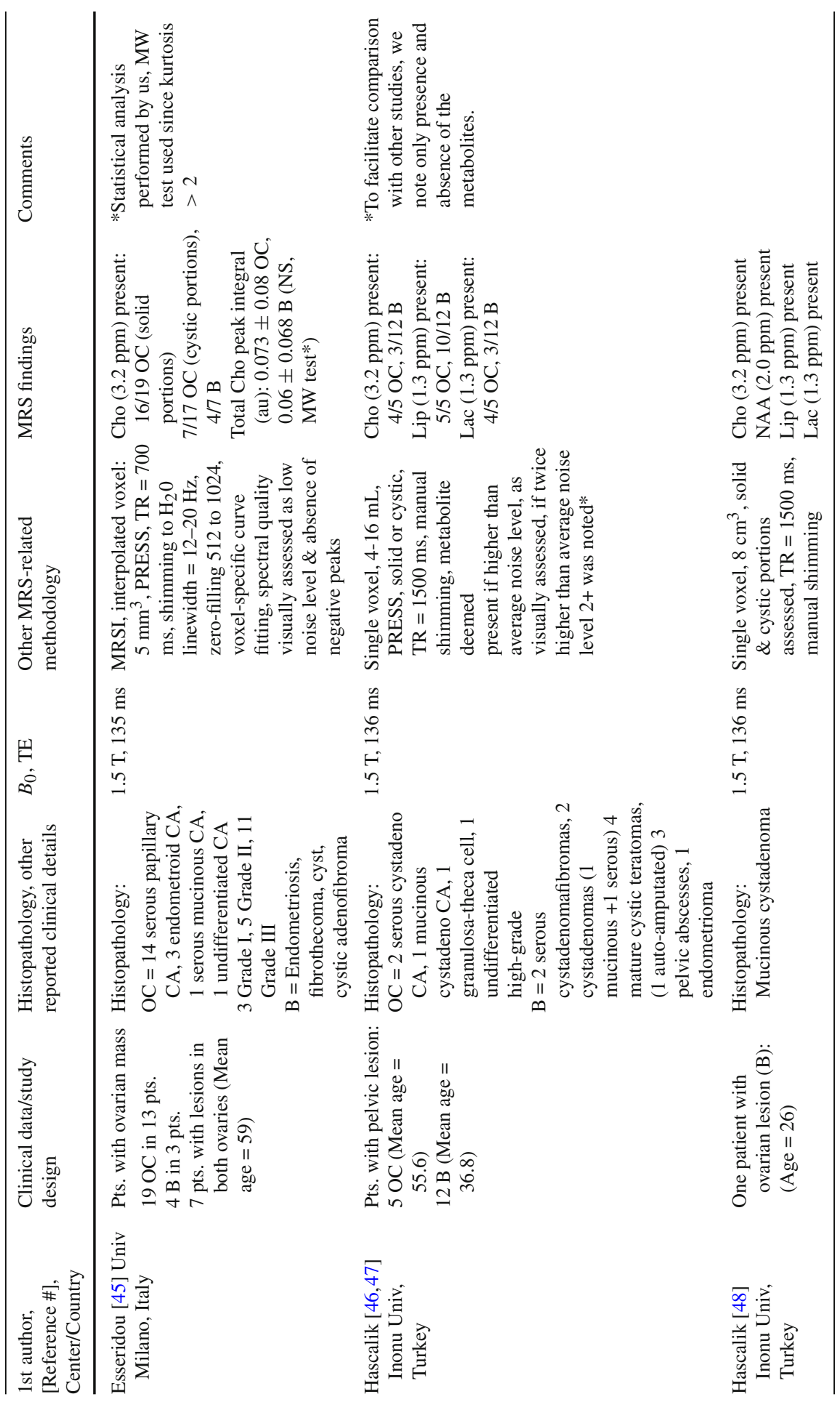




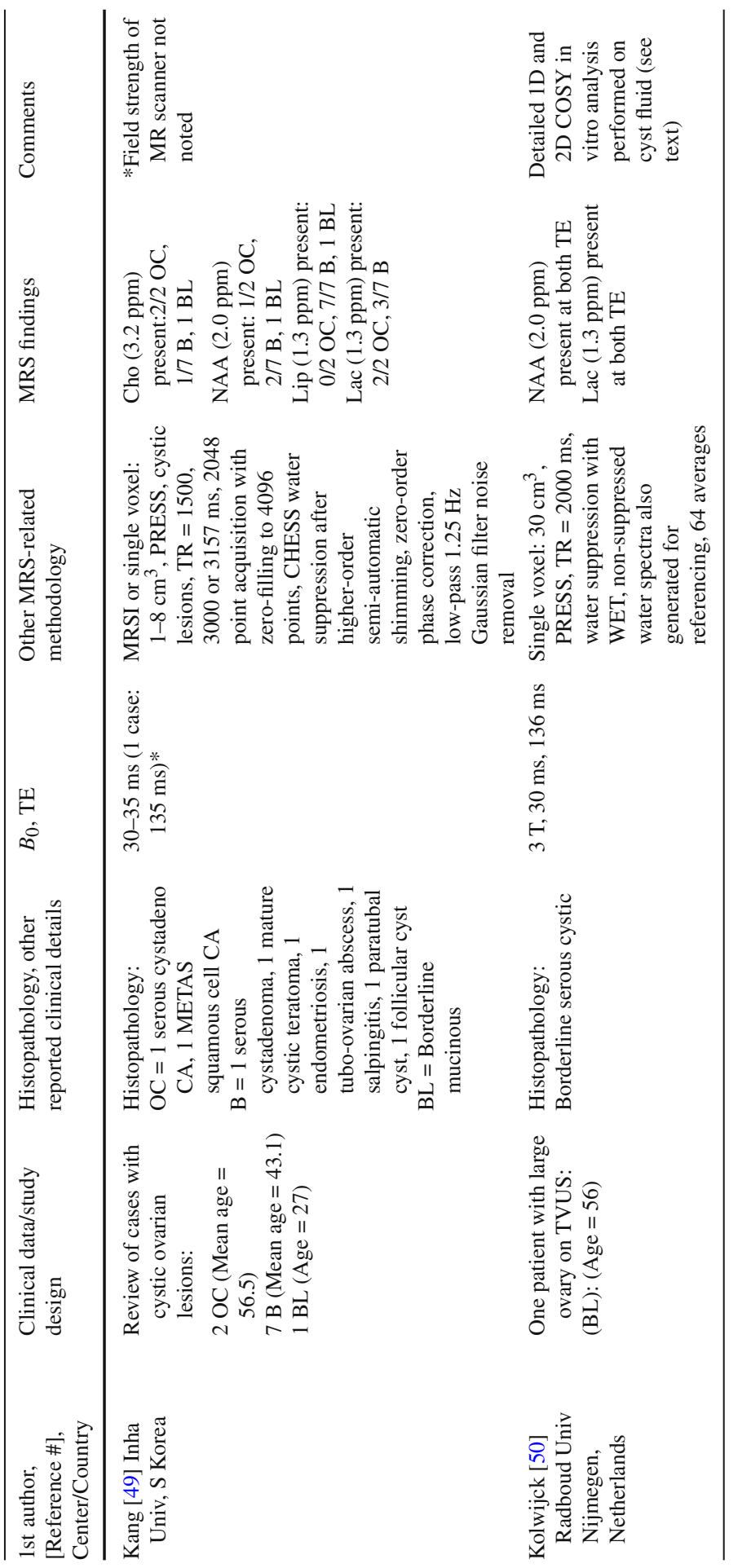




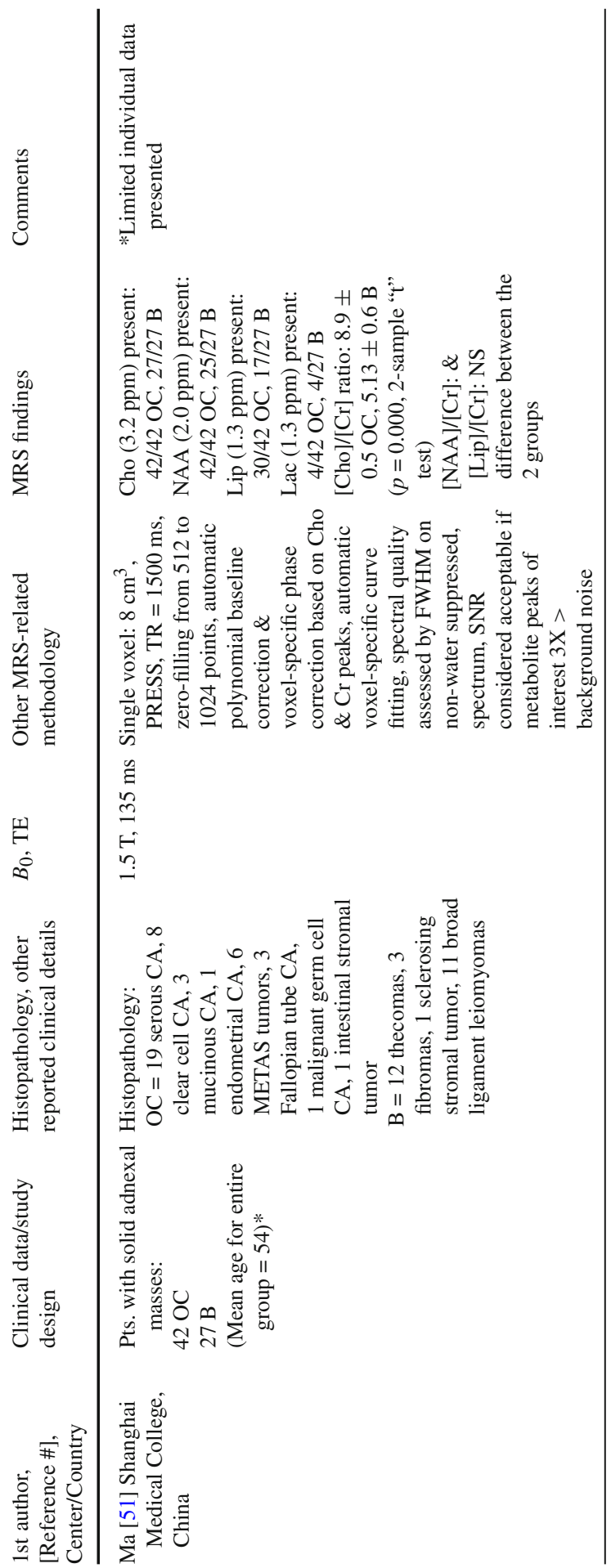




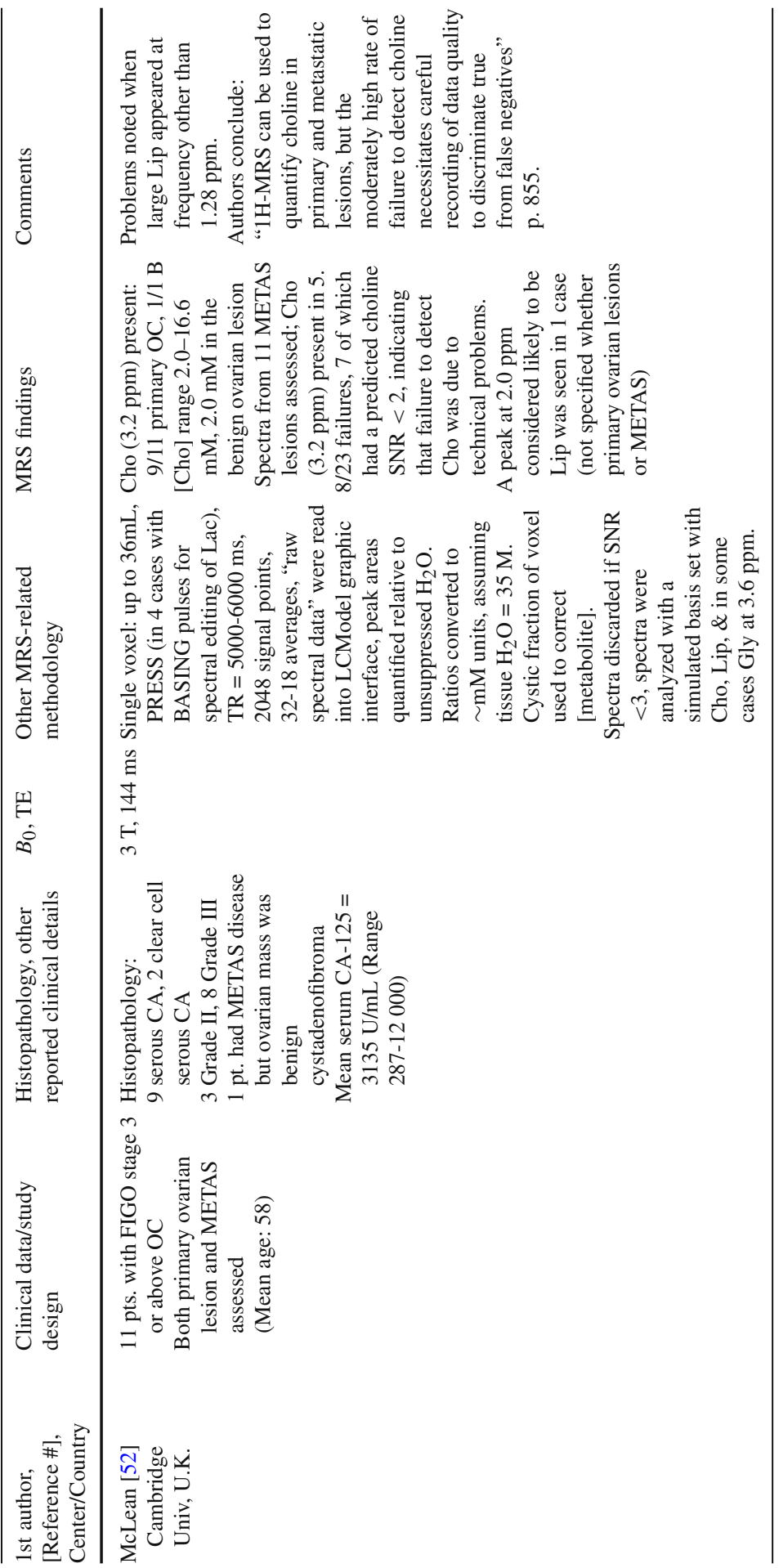




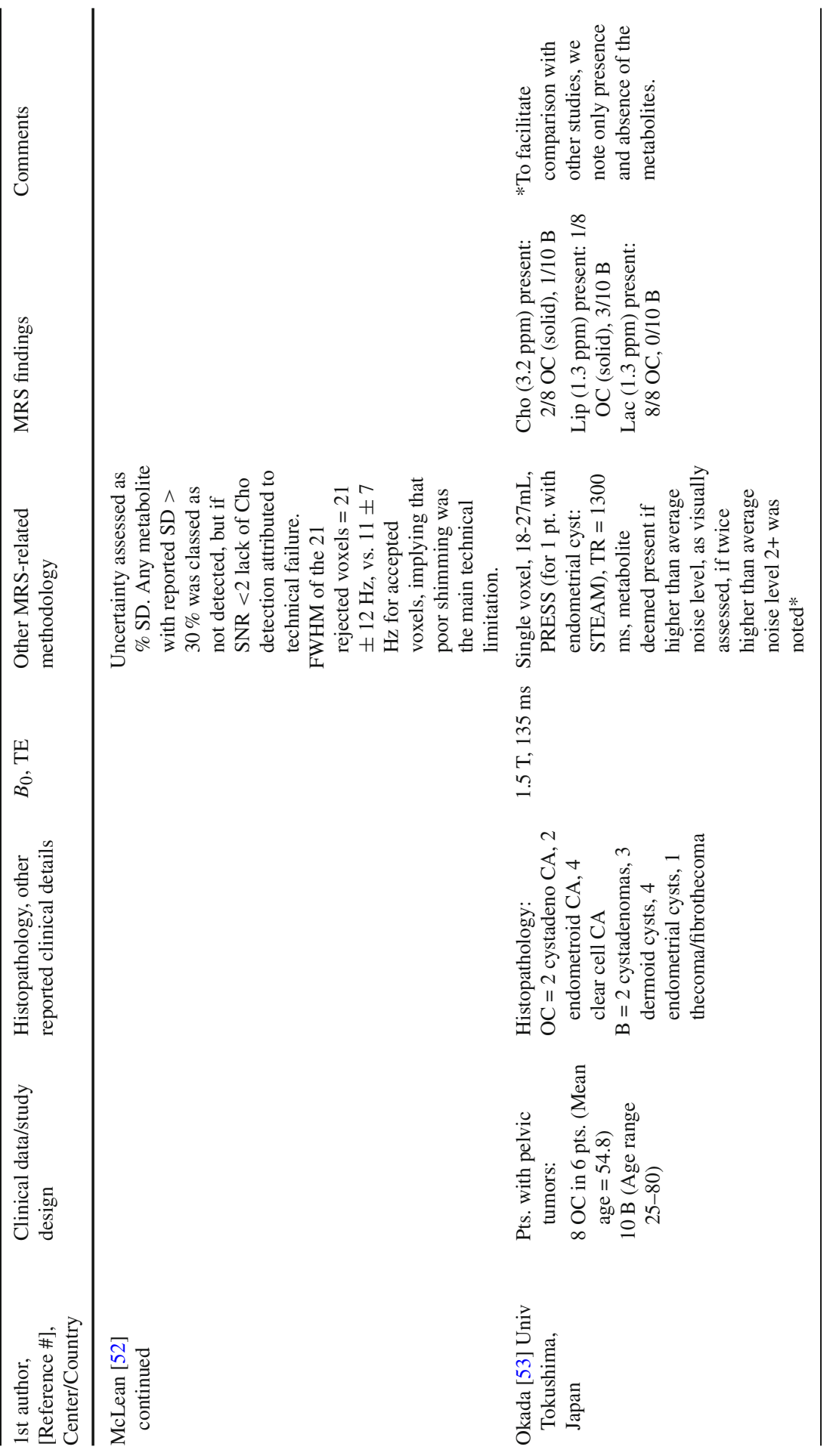




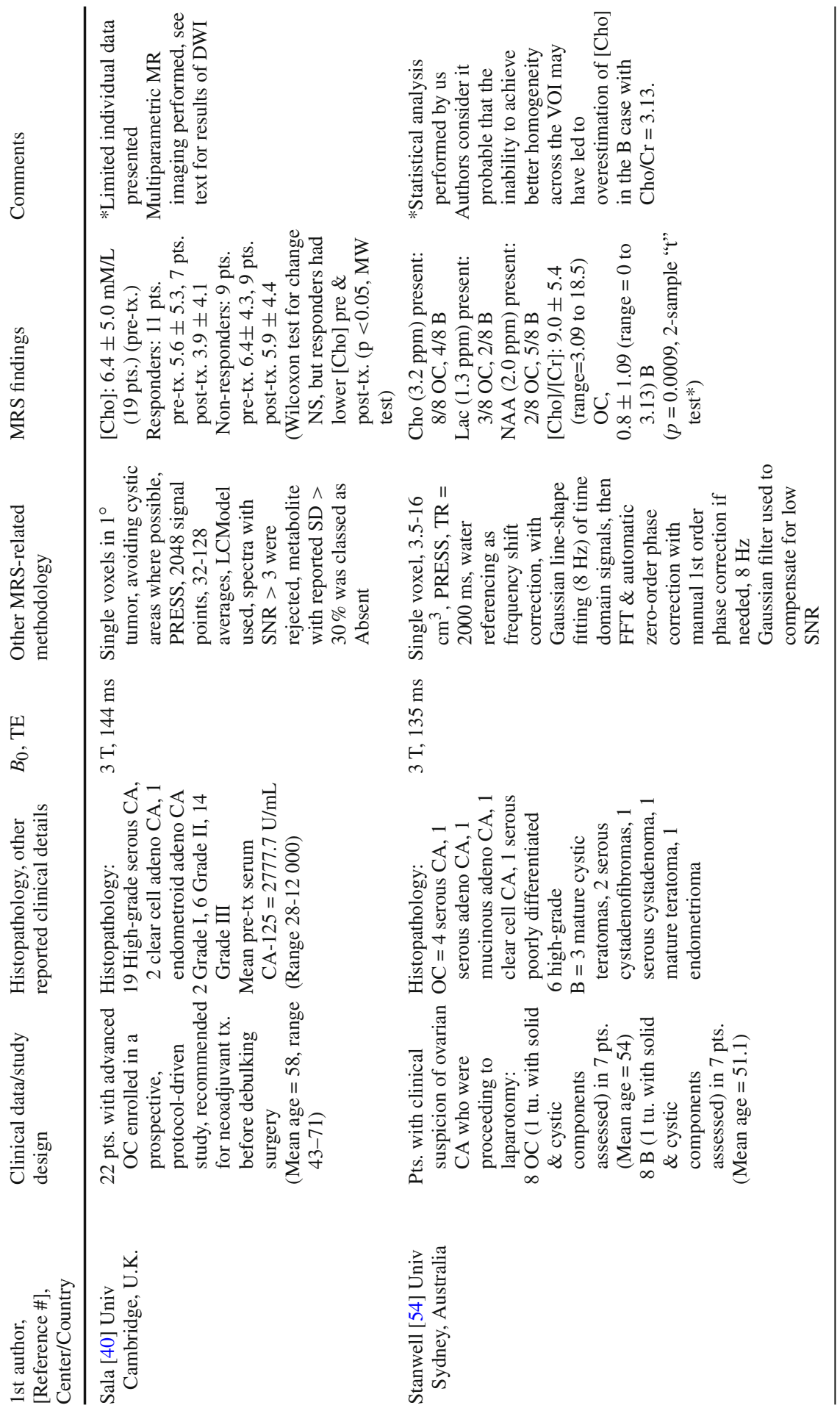




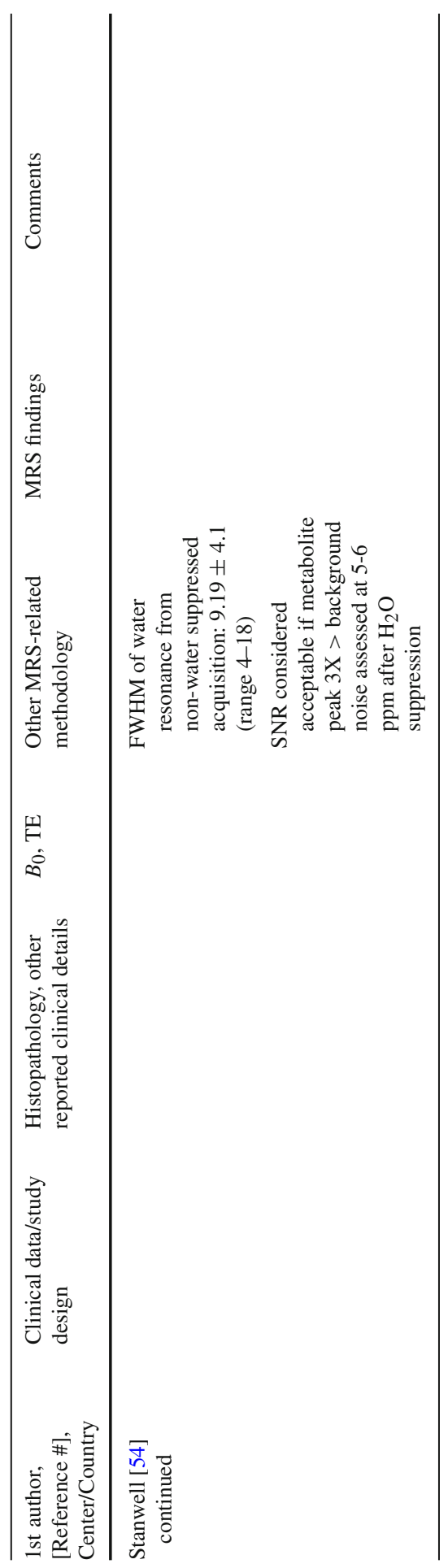




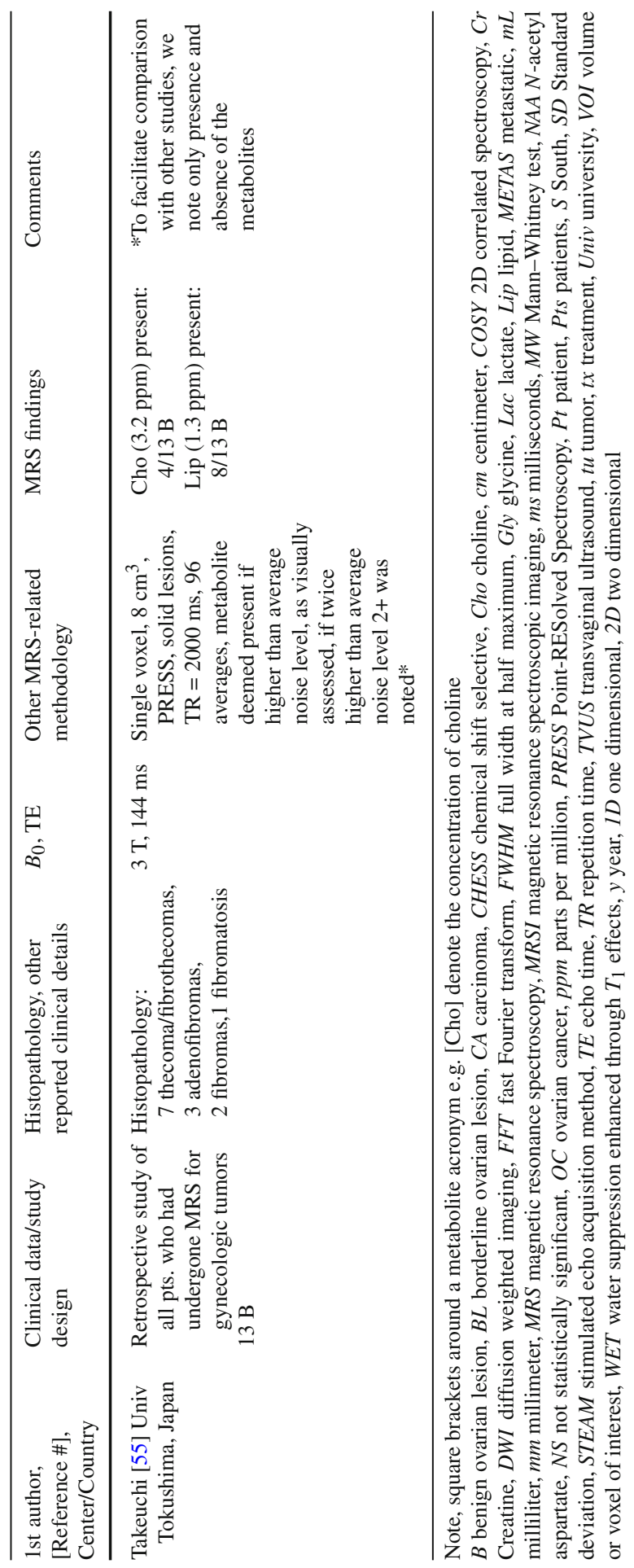




\section{References}

1. J.D. Seidman, B.G. Wang, Evaluation of normal-sized ovaries associated with primary peritoneal serous carcinoma for possible precursors of ovarian serous carcinoma. Gynecol. Oncol. 106, 201-206 (2007)

2. M. Åkeson, A. Jakobsen, B. Zetterqvist, E. Holmberg, M. Brannström, G. Horvath, A population-based 5-year cohort study of epithelial ovarian cancer in western Sweden: 10-year survival and prognostic factors. Int. J. Gynecol. Cancer 19, 116-123 (2009)

3. D. Kisić-Tepavčević, B. Matejić, T. Gazibara, T. Pekmezović, Trends and patterns of ovarian cancer mortality in Belgrade, Serbia: a joinpoint regression analysis. Int. J. Gynecol. Cancer 21, 1018-1023 (2011)

4. A. Sharifian, M. Amin Pourhoseingholi, M. Norouzinia, M. Vahedi, Ovarian cancer in Iranian women, a trend analysis of mortality and incidence. Asian Pac. J. Cancer Prev. 15, 10787-10790 (2014)

5. J.M. Schildkraut, A.J. Alberg, E.V. Bandera, J. Barnholtz-Sloan, M. Bondy, M.L. Cote, E. Funkhouser, E. Peters, A.G. Schwartz, P. Terry, K. Wallace, L. Akushevich, F. Wang, S. Crankshaw, P.G. Moorman, A multi-center population-based case-control study of ovarian cancer in African-American women: the African American Cancer Epidemiology Study (AACES). BMC Cancer 14, 688 (2014)

6. A. Chetrit, G. Hirsh-Yechezkel, Y. Ben-David, F. Lubin, E. Friedman, S. Sadetzki, Effect of BRCA1/2 mutations on long-term survival of patients with invasive ovarian cancer: the National Israeli Study of Ovarian Cancer. J. Clin. Oncol. 26, 20-25 (2008)

7. N. Wentzensen, S. Wacholder, Talc use and ovarian cancer: epidemiology between a rock and a hard place. J. Natl. Cancer Inst. 106, (2014). doi:10.1093/jnci/dju260

8. E.J. Mališić, R.N. Janković, K.V. Jakovljević, S. Radulović, Association of TP53 codon 72 polymorphism with susceptibility to ovarian carcinomas in Serbian women. Eur. J. Obstet. Gynceol. Reprod. Biol. 166, 90-93 (2013)

9. L.E. Minion, J.S. Dolinsky, D.M. Chase, C.L. Dunlop, E.C. Chao, B.J. Monk, Hereditary predisposition to ovarian cancer, looking beyond BRCA1/BRCA2. Gynecol. Oncol. 137, 86-92 (2015)

10. P.D.P. Pharoah, The potential for risk stratification in the management of ovarian cancer risk. Int. J. Gynecol. Cancer 22, S16-S17 (2012)

11. F. Salehi, L. Dunfield, K. Phillips, D. Krewski, B. Vanderhyden, Risk factors for ovarian cancer: an overview with emphasis on hormonal factors. J. Toxicol. Environ. Health 11, 301-321 (2008)

12. J.O. Schorge, S.C. Modesitt, R.L. Coleman, D.E. Cohn, N.D. Kauff, L.R. Duska, T.J. Herzog, SGO White Paper on ovarian cancer: etiology, screening and surveillance. Gyncol. Oncol. 119, 7-17 (2010)

13. N. Wentzensen, B. Trabert, Hormone therapy: short-term relief, long-term consequences. Lancet $\mathbf{3 8 5}$, 1806-1808 (2015)

14. Å. Klint, L. Tryggvadottir, F. Bray, M. Gislum, T. Hakulinen, H. Storm, M. Enghol, Trends in the survival of patients diagnosed with cancer in female genital organs in Nordic countries. Acta Oncol. 49, 632-643 (2010)

15. P.M. Webb, Environmental (nongenetic) factors in gynecological cancers: update and future perspectives. Future Oncol. 11, 295-307 (2015)

16. P. Bhatti, K.L. Cushing-Haugen, K.G. Wicklund, J. Doherty, M.A. Rossing, Nightshift work and risk of ovarian cancer. Occup. Environ. Med. 70, 231-237 (2013)

17. H. Jim, H.-Y. Lin, J. Tyrer, K. Lawrenson, J. Dennis, G. Chornokur, Z. Chen, A. Chen, J. Permuth-Wey, K. Aben, H. Anton-Culver, N. Antonenkova, F. Bruinsma, E.V. Bandera, Y.T. Bean, M.W. Beckmann, M. Bisogna, L. Bjorge, N. Bogdanova, L.A. Brinton, A. Brooks-Wilson, C.H. Bunker, R. Butzow, I.G. Campbell, K. Carty, J. Chang-Claude, L.S. Cook, D.W. Cramer, J.M. Cunningham, C. Cybulski, A. Dansonka-Mieszkowska, A. du Bois, E. Despierre, W. Sieh, J.A. Doherty, T. Dörk, M. Dürst, D.F. Easton, D.M. Eccles, R.P. Edwards, A.B. Ekici, P.A. Fasching, B.L. Fridley, Yu.-T. Gao, A. Gentry-Maharaj, G.G. Giles, R. Glasspool, M.T. Goodman, J. Gronwald, P. Harter, H.N. Hasmad, A. Hein, F. Heitz, M.A.T. Hildebrandt, P. Hillemanns, C.K. Hogdall, E. Hogdall, S. Hosono, E.S. Iversen, A. Jakubowska, A. Jensen, B.-T. Ji, B.Y. Karlan, M. Kellar, L.A. Kiemeney, C. Krakstad, S.K. Kjaer, J. Kupryjanczyk, R.A. Vierkant, D. Lambrechts, S. Lambrechts, N.D. Le, A.W. Lee, S. Lele, A. Leminen, J. Lester, D.A. Levine, D. Liang, B.K. Lim, J. Lissowska, K. Lu, J. Lubinski, L. Lundvall, L.F.A.G. Massuger, K. Matsuo, V. McGuire, J.R. McLaughlin, I. McNeish, U. Menon, R.L. Milne, F. Modugno, L. Thomsen, K.B. Moysich, R.B. Ness, H. Nevanlinna, U. Eilber, K. Odunsi, S. Olson, I. Orlow, S. Orsulic, J. Paul, C.L. Pearce, T. Pejovic, L.M. Pelttari, M.C. Pike, E.M. Poole, E. Schernhammer, H.A. Risch, B. Rosen, M. Rossing, J.H. Rothstein, A. Rudolph, I.B. Runnebaum, 
I.K. Rzepecka, H.B. Salvesen, I. Schwaab, X.-O. Shu, Y.B. Shvetsov, N. Siddiqui, H. Song, M.C. Southey, B. Spiewankiewicz, L. Sucheston-Campbell, S. Teo, K.L. Terry, P.J. Thompson, I.L. Tangen, S. Tworoger, A.M. van Altena, I. Vergote, C. Walsh, S. Wang-Gohrke, P. Weber, N. Wentzensen, A.S. Whittemore, K.G. Wicklund, L.R. Wilkens, A.H. Wu, X. Wu, Y.-L. Woo, H. Yang, Wei Zheng, A. Ziogas, E. Amankwah, A. Berchuck, G. Chenevix-Trench (on behalf of the AOCS management group), J.M. Schildkraut, L.E. Kelemen, S.J. Ramus, A.N.A. Monteiro, E.L. Goode, S.A. Narod, S.A. Gayther, P.D.P. Pharoah, T.A. Sellers, C.M. Phelan, Common genetic variation in circadian rhythm genes and risk of epithelial ovarian cancer (EOC). J. Genet. Genome. Res. 2, 017 (2015)

18. P. Mohaghegh, A.G. Rockall, Imaging strategy for early ovarian cancer: characterization of adnexal masses with conventional and advanced imaging techniques. Radiographics 32, 1751-1773 (2012)

19. G. Chornokur, E. Armankwah, J. Schildkraut, C. Phelan, Global ovarian cancer health disparities. Gynecol. Oncol. 129, 258-264 (2013)

20. M. Andersen, K. Lowe, B. Goff, Value of symptom-triggered diagnostic evaluation for ovarian cancer. Obstet. Gynecol. 123, 73-79 (2014)

21. I.J. Jacobs, U. Menon, A. Ryan, A. Gentry-Maharaj, M. Burnell, J.K. Kalsi, N. Amso, S. Apostolidou, E. Benjamin, D. Cruickshank, D.N. Crump, S. Davies, A. Dawnay, S. Dobbs, G. Fletcher, J. Ford, K. Godfrey, R. Gunu, M. Habib, R. Hallett, J. Herod, H. Jenkins, C. Karpinskyj, S. Leeson, S. Lewis, W. Liston, A. Lopes, T. Mould, J. Murdoch, D. Oram, D. Rabideau, K. Reynolds, I. Scott, M. Seif, A. Sharma, N. Singh, J. Taylor, F. Warburton, M. Widschwendter, K. Williamson, R. Woolas, L. Fallowfield, A. McGuire, S. Campbell, M. Parmar, S. Skates, Ovarian cancer screening and mortality in the UK Collaborative Trial of Ovarian Cancer Screening (UKCTOCS): A randomised controlled trial. Lancet 387, 945-956 (2016)

22. E. Partridge, R.T. Greenlee, T.L. Riley, J. Commins, L. Ragard, J.-L. Xu, S. Buys, P.C. Prorok, M.N. Fouad, Assessing the risk of ovarian malignancy in asymptomatic women with abnormal CA-125 and transvaginal ultrasound scans in the Prostate, Lung, Colorectal, and Ovarian Screening Trial. Obstet. Gynecol. 121, 25-31 (2013)

23. H. Kobayashi, Y. Yamada, T. Sado, M. Sakata, S. Yoshida, R. Kawaguchi, S. Kanayama, H. Shigetomi, S. Haruta, Y. Tsuji, S. Ueda, T. Kitanaka, A randomized study of screening for ovarian cancer: a multicenter study in Japan. Int. J. Gynecol. Cancer 18, 414-420 (2008)

24. H. Kobayashi, Y. Yamada, T. Sado, M. Sakata, S. Yoshida, R. Kawaguchi, S. Kanayama, H. Shigetomi, S. Haruta, Y. Tsuji, S. Ueda, T. Kitanaka, H. Oi, Prevalence of ovarian cancer among women with a CA125 level of $35 \mathrm{U} / \mathrm{ml}$ or less. Gynecol. Obstet. Invest. 65, 133-138 (2008)

25. C.M. Castro, H. Im, C. Le, H. Lee, R. Weissleder, M.J. Birrer, Exploring alternative ovarian cancer biomarkers using innovative nanotechnology strategies. Cancer Metastasis Rev. 34, 75-82 (2015)

26. S. Kadija, A. Stefanović, K. Jeremić, M.M. Radojević, Lj Nikolić, I. Marković, J. Atanacković, The utility of human epididymal protein 4, cancer antigen 125, and risk for malignancy algorithm in ovarian cancer and endometriosis. Int. J. Gynecol. Cancer 22, 238-244 (2012)

27. V. Nossov, M. Amneus, F. Su, J. Lang, J.M. Janco, S.T. Reddy, R. Farias-Eisner, The early detection of ovarian cancer: from traditional methods to proteomics: can we really do better than serum CA-125? Am. J. Obstet. Gynecol. 199, 215-223 (2008)

28. M.K. Pal, M. Rashid, M. Bisht, Multiplexed magnetic nanoparticle-antibody conjugates (MNPs-ABS) based prognostic detection of ovarian cancer biomarkers, CA-125, $\beta$-2M and ApoA1 using fluorescence spectroscopy with comparison of surface plasmon resonance (SPR) analysis. Biosens. Bioelectron. 73, 146-152 (2015)

29. C.M. Slupsky, H. Steed, T.H. Wells, K. Dabbs, A. Schepansky, V. Capstick, W. Faught, M.B. Sawyer, Urine metabolite analysis offers potential early diagnosis of ovarian and breast cancers. Clin. Cancer Res. 16, 5835-5841 (2010)

30. G. Sölétormos, M.J. Duffy, S. Othman Abu Hassan, R.H.M. Verheijen, B. Tholander, R.C. Bast, K.N. Gaarenstroom, C.M. Sturgeon, J.M. Bonfrer, P.H. Petersen, H. Troonen, G. Torre, J. Kanty Kulpa, M.K. Tuxen, R. Molina, Clinical use of cancer biomarkers in epithelial ovarian cancer: updated guidelines from the European Group on Tumor Markers. Int. J. Gynecol. Cancer 26, 43-51 (2016)

31. U. Menon, M. Griffin, A. Gentry-Maharaj, Ovarian cancer screening - current status, future directions. Gynecol. Oncol. 132, 490-495 (2014)

32. K.L. Taylor, R. Shelby, E. Gelmann, C. McGuire, Quality of life and trial adherence among participants in the prostate, lung, colorectal, and ovarian cancer screening trial. J. Natl. Cancer Inst. 96, 1083-1094 (2004) 
33. A. Slomski, Screening women for ovarian cancer still does more harm than good. J. Am. Med. Assoc. 307, 2474-2475 (2012)

34. V.A. Moyer, Screening for ovarian cancer: U.S. Preventive Services Task Force reaffirmation recommendation. Ann. Intern. Med. 157, 900-904 (2012)

35. E. Sala, A.G. Rockall, S.J. Freeman, D.G. Mitchell, C. Reinhold, The added role of MR imaging in treatment stratification of patients with gynecologic malignancies: What the radiologist needs to know. Radiology 266, 717-740 (2013)

36. I. Imaoka, T. Araki, M. Takeuchi, MRI of the female genitourinary tract, in Magnetic Resonance Volume 3, Comprehensive Biomedical Physics, ed. by Dž. Belkić, K. Belkić (Elsevier, Amsterdam, 2014), pp. 221-240

37. K. Kinkel, Y. Lu, A. Mehdizade, M.-F. Pelte, H. Hricak, Indeterminate ovarian mass at US: incremental value of second imaging test for characterization. Radiology 236, 85-94 (2005)

38. S. Zhao, J. Qiang, G. Zhang, F. Ma, S. Cai, H. Li, L. Wang, Diffusion-weighted MR imaging for differentiating borderline from malignant epithelial tumours of the ovary: pathological correlation. Eur. Radiol. 24, 2292-2299 (2014)

39. J. Djokić-Kovač, M. Terzić, M. Mirković, B. Banko, A. Dikić-Rom, R. Maksimović, Endometrioid adenocarcinoma of the ovary: MRI findings with emphasis on diffusion-weighted imaging for the differentiation of ovarian tumors. Acta Radiol. 57, 758-766 (2016)

40. E. Sala, M.Y. Kataoka, A.N. Priest, A.B. Gill, M.A. McLean, I. Joubert, M.J. Graves, R.A.F. Crawford, M. Jimenez-Linan, H.M. Earl, C. Hodgkin, J.R. Griffiths, D.J. Lomas, J.D. Brenton, Advanced ovarian cancer: multiparametric MR imaging demonstrates response- and metastasis-specific effects. Radiology 263, 149-159 (2012)

41. D. Hanahan, R.A. Weinberg, Hallmarks of cancer: the next generation. Cell 144, 646-674 (2011)

42. M.F. Kircher, H. Hricak, S.M. Larson, Molecular imaging for personalized cancer care. Mol. Oncol. 6, 182-195 (2012)

43. S.J. Booth, M.D. Pickles, L.W. Turnbull, In vivo magnetic resonance spectroscopy of gynaecological tumors at 3.0 tesla. Br. J. Obstet. Gynaecol. 116, 300-303 (2009)

44. S.W. Cho, S.G. Cho, J.H. Lee, H.-J. Kim, M.H. Lim, J.H. Kim, C.H. Suh, In vivo proton magnetic resonance spectroscopy in adnexal lesions. Korean J. Radiol. 3, 105-112 (2002)

45. A. Esseridou, G. Di Leo, L.M. Sconfienza, V. Caldiera, F. Raspagliesi, B. Grijuela, F. Hanozet, F. Podo, F. Sardanelli, In vivo detection of choline in ovarian tumors using 3D MRS. Investig. Radiol. 46, 377-382 (2011)

46. S. Hascalik, O. Celik, G. Erdem, Magnetic resonance spectral analysis of ovarian teratomas. Int. J. Gynecol. Obstet. 90, 152-152 (2005)

47. S. Hascalik, O. Celik, K. Sarac, M.M. Meydanli, A. Alkan, B. Mizrak, Metabolic changes in pelvic lesions: findings at proton MR spectroscopic imaging. Gynecol. Obstet. Invest. 60, 121-127 (2005)

48. S. Hascalik, O. Celik, K. Sarac, A. Alkan, B. Mizrak, Clinical significant of $N$-acetyl-1-aspartate resonance in ovarian mucinous cystadenoma. Int. J. Gyncol. Cancer 16, 423-447 (2006)

49. Y.H. Kang, M.Y. Kim, K.T. Kim, Y.J. Kim, C.H. Suh, J.M. Kim, S.O. Hwang, S. Park, J.Y. Cho, ${ }^{1}$ H magnetic resonance spectroscopy of cystic ovarian lesions. J. Korean Soc. Magn. Reson. Med. 17, 326-333 (2013)

50. E. Kolwijck, U.F. Engelke, M. van der Graaf, A. Heerschap, J. Henk, H.J. Blom, M. Hadfoune, W.A. Buurman, L.F. Massuger, R.A. Wevers, N-acetyl resonances in in vivo and in vitro NMR spectroscopy of cystic ovarian tumors. NMR Biomed. 22, 1093-1099 (2009)

51. F.H. Ma, J.W. Qiang, S.Q. Cai, S.H. Zhao, G.F. Zhang, Y.M. Rao, MR spectroscopy for differentiating benign from malignant solid adnexal tumors. Am. J. Roentgenol. 204, W724-W730 (2015)

52. M.A. McLean, A.N. Priest, I. Joubert, D.J. Lomas, M.Y. Kataoka, H. Earl, R. Crawford, J.D. Brenton, J.R. Griffiths, E. Sala, Metabolic characterization of primary and metastatic ovarian cancer by $1 \mathrm{H}-\mathrm{MRS}$ in vivo at 3T. Magn. Reson. Med. 62, 855-861 (2009)

53. T. Okada, M. Harada, K. Matsuzaki, H. Nishitani, T.J. Aono, Evaluation of female intrapelvic tumors by clinical proton MR spectroscopy. J. Magn. Reson. Imaging 13, 912-917 (2001)

54. P. Stanwell, P. Russell, J. Carter, S. Pather, S. Heintze, C. Mountford, Evaluation of ovarian tumors by proton magnetic resonance spectroscopy at three tesla. Invest. Radiol. 43, 745-751 (2008)

55. M. Takeuchi, K. Matsuzaki, M. Harada, Preliminary observations and diagnostic value of lipid peak in ovarian thecomas/fibrothecomas using in vivo proton MR spectroscopy at 3T. J. Magn. Reson. Imaging 36, 907-911 (2012) 
56. I.C. Smith, D.E. Blandford, Diagnosis of cancer in humans by $1 \mathrm{H}$ NMR of tissue biopsies. Biochem. Cell. Biol. 76, 472-476 (1998)

57. J.C. Wallace, G.P. Raaphorst, R.L. Somorjai, C.E. Ng, M. Fung Kee Fung, M. Senterman, I.C. Smith, Classification of $1 \mathrm{H}$ MR spectra of biopsies from untreated and recurrent ovarian cancer using linear discriminant analysis. Magn. Reson. Med. 38, 569-576 (1997)

58. L.F.A.G. Massuger, P.B.J. van Vierzen, U. Engelke, A. Heerschap, R. Wevers, $1 \mathrm{H}$ magnetic resonance spectroscopy. A new technique to discriminate benign from malignant ovarian tumors. Cancer $\mathbf{8 2}$, $1726-1730$ (1998)

59. E.A. Boss, S.H. Moolenaar, L.F. Massuger, H. Boonstra, U.F. Engelke, J.G. de Jong, R.A. Wevers, High-resolution proton nuclear magnetic resonance spectroscopy of ovarian cyst fluid. NMR Biomed. 13, 297-30 (2000)

60. E. Kolwijck, R.A. Wevers, U.F. Engelke, J. Woudenberg, J. Bulten, H.J. Blom, L.F.A.G. Massuger, Ovarian cyst fluid of serous ovarian tumors contains large quantities of the brain amino acid $N$ acetylaspartate. PLoS One 5, e10293 (2010)

61. D. Ben Sellem, K. Elbayed, A. Neuville, F.-M. Moussallieh, G. Lang-Averous, M. Piotto, J.-P. Bellocq, I.J. Namer, Metabolomic characterization of ovarian epithelial carcinomas by HRMAS-NMR spectroscopy. J. Oncol. (2011). doi:10.1155/2011/174019

62. M. Kyriakides, N. Rama, J. Sidhu, H. Gabra, H.C. Keun, M. El-Bahrawy, Metabonomic analysis of ovarian tumour cyst fluid by proton nuclear magnetic resonance spectroscopy. Oncotarget 7, 7216-7226 (2016)

63. Y. Abramov, S. Carmi, S.O. Anteby, I. Ringel, Ex vivo $1 \mathrm{H}$ and $31 \mathrm{P}$ magnetic resonance spectroscopy as a means for tumor characterization in ovarian cancer patients. Oncol. Rep. 29, 321-328 (2013)

64. E. Iorio, D. Mezzanzanica, P. Alberti, F. Spadaro, C. Ramoni, S. D’Ascenzo, D. Millimaggi, A. Pavan, V. Dolo, S. Canavari, F. Podo, Alterations of choline phospholipid metabolism in ovarian tumor progression. Cancer Res. 65, 9369-9376 (2005)

65. K. Glunde, J. Jiang, S.A. Moestue, I.S. Gribbestad, MRS/MRSI guidance in molecular medicine: targeting choline and glucose metabolism. NMR Biomed. 24, 673-690 (2011)

66. Dž. Belkić, K. Belkić, Mathematical modeling applied to an NMR problem in ovarian cancer detection. J. Math. Chem. 43, 395-425 (2008)

67. Dž. Belkić, Quantum-Mechanical Signal Processing and Spectral Analysis (Institute of Physics Publishing, Bristol, 2005)

68. Dž. Belkić, Strikingly stable convergence of the Fast Padé transform (FPT) for high-resolution parametric and non-parametric signal processing of Lorentzian and non-Lorentzian spectra. Nucl. Instrum. Methods Phys. Res. A 525, 366-371 (2004)

69. Dž. Belkić, Exact quantification of time signals in Padé-based magnetic resonance spectroscopy. Phys. Med. Biol. 51, 2633-2670 (2006)

70. Dž. Belkić, Analytical continuation by numerical means in spectral analysis using the fast Padé transform (FPT). Nucl. Instrum. Methods Phys. Res. A 525, 372-378 (2004)

71. K. Belkić, Resolution performance of the fast Padé transform: Potential advantages for magnetic resonance spectroscopy in ovarian cancer diagnostics. Nucl. Instrum. Methods Phys. Res A 580, 874880 (2007)

72. Dž. Belkić, Exact signal-noise separation by Froissart doublets in the fast Padé transform for magnetic resonance spectroscopy. Adv. Quantum Chem. 56, 95-179 (2009)

73. Dž. Belkić, K. Belkić, Magnetic resonance spectroscopy with high-resolution and exact quantification in the presence of noise for improving ovarian cancer detection. J. Math. Chem. 50, 2558-2576 (2012)

74. Dž. Belkić, K. Belkić, Resolution enhancement as a key step towards clinical implementation of Padéoptimized magnetic resonance spectroscopy for diagnostic oncology. J. Math. Chem. 51, 2608-2637 (2013)

75. Dž. Belkić, K. Belkić, Strategic steps for advanced molecular imaging with magnetic resonance-based diagnostic modalities. Technol. Cancer Res. Treat. 14, 119-142 (2015)

76. Dž. Belkić, K. Belkić, How the fast Padé transform handles noise for MRS data from the ovary. J. Math. Chem. 54, 149-185 (2016)

77. Dž. Belkić, K. Belkić, Signal Processing in Magnetic Resonance Spectroscopy with Biomedical Applications (Taylor \& Francis, London, 2010)

78. Dž. Belkić, K. Belkić, The general concept of signal-noise separation (SNS). J. Math. Chem. 45, 563-597 (2009) 
79. Dž. Belkić, K. Belkić, Iterative averaging of spectra as a powerful way of suppressing spurious resonances in signal processing. J. Math. Chem. (2016). doi:10.1007/s10910-016-0693-9

80. Dž. Belkić, Exponential convergence rate (the spectral convergence) of the fast Padé transform for exact quantification in magnetic resonance spectroscopy. Phys. Med. Biol. 51, 6483-6512 (2006)

81. Dž. Belkić, Machine accurate quantification in magnetic resonance spectroscopy. Nucl. Instrum. Methods Phys. Res. A 580, 1034-1040 (2007)

82. Dž. Belkić, K. Belkić, Exact quantification of time signals from magnetic resonance spectroscopy by the fast Padé transform with applications to breast cancer diagnostics. J. Math. Chem. 45, 790-818 (2009)

83. Dž. Belkić, K. Belkić, Unequivocal resolution of multiplets in MR spectra for prostate cancer diagnostics achieved by the fast Padé transform. J. Math. Chem. 45, 819-858 (2009)

84. Dž. Belkić, K. Belkić, Padé-optimization of noise-corrupted magnetic resonance spectroscopic time signals from fibroadenoma of the breast. J. Math. Chem. 52, 2680-2713 (2014)

85. Dž. Belkić, K. Belkić, Optimized spectral analysis in magnetic resonance spectroscopy for early tumor diagnostics. J. Phys. Conf. Ser. 565, 012002 (2014). doi:10.1088/1742-6596/565/1/012002

86. Dž. Belkić, K. Belkić, Proof-of-the-concept study on mathematically optimized magnetic resonance spectroscopy for breast cancer diagnostics. Technol. Cancer Res. Treat. 14, 277-297 (2015)

87. Dž. Belkić, K. Belkić, Mathematically-optimized magnetic resonance spectroscopy in breast cancer diagnostics. J. Math. Chem. 54, 186-230 (2016)

88. Dž. Belkić, K. Belkić, The fast Padé transform for noisy magnetic resonance spectroscopic data from the prostate. J. Math. Chem. 54, 707-764 (2016)

89. Dž. Belkić, K. Belkić, Quantification by the fast Padé transform of magnetic resonance spectroscopic data encoded at 1.5 T. J. Math. Chem. 54, 602-655 (2016)

90. Dž. Belkić, K. Belkić, Improving the diagnostic yield of magnetic resonance spectroscopy for pediatric brain tumors through mathematical optimization. J. Math. Chem. 54, 1461-1513 (2016)

91. Dž. Belkić, K. Belkić, High-resolution quantum-mechanical signal processing for in vivo NMR spectroscopy. Adv. Quantum Chem. doi:10.1016/bs.aiq.2016.06.004

92. Dž. Belkić, K. Belkić, The potential for practical improvements in cancer diagnostics by mathematically-optimized magnetic resonance spectroscopy. J. Math. Chem. 49, 2408-2440 (2011)

93. Dž. Belkić, Error analysis through residual frequency spectra in the fast Padé transform (FPT). Nucl. Instrum. Methods Phys. Res A 525, 379-386 (2004)

94. Dž. Belkić, K. Belkić, The fast Padé transform in magnetic resonance spectroscopy for potential improvements in early cancer diagnostics. Phys. Med. Biol. 50, 4385-4408 (2005)

95. Dž. Belkić, K. Belkić, In vivo magnetic resonance spectroscopy by the fast Padé transform. Phys. Med. Biol. 51, 1049-1075 (2006)

96. K. Belkić, M. Cohen, M. Márquez, M. Mints, B. Wilczek, A.H. Berman, E. Castellanos, M. Castellanos, Screening of high-risk groups for breast and ovarian cancer in Europe: a focus on the Jewish population. Oncol. Rev. 4, 233-267 (2010)

97. E. Kolwijck, C. Lybol, J. Bulten, J.H.A. Vollebergh, R.A. Wevers, L.F.A.G. Massuger, Prevalence of cysts in epithelial ovarian cancer. Eur. J. Obstet. Gynecol. 151, 96-100 (2010)

98. S. Harlap, S.H. Olson, R.R. Barakat, T.A. Caputo, S. Forment, A.J. Jacobs, C. Nakraseive, X. Xue, Diagnostic X-rays and risk of epithelial ovarian carcinoma in Jews. Ann. Epidemiol. 12, 426-434 (2002) 